\title{
Mechanisms involved in the control of breathing before and after birth
}

Citation for published version (APA):

Kuipers, I. M. (1995). Mechanisms involved in the control of breathing before and after birth. [Doctoral Thesis, Maastricht University]. Rijksuniversiteit Limburg. https://doi.org/10.26481/dis.19950420ik

Document status and date:

Published: 01/01/1995

DOI:

10.26481/dis.19950420ik

Document Version:

Publisher's PDF, also known as Version of record

\section{Please check the document version of this publication:}

- A submitted manuscript is the version of the article upon submission and before peer-review. There can be important differences between the submitted version and the official published version of record.

People interested in the research are advised to contact the author for the final version of the publication, or visit the DOI to the publisher's website.

- The final author version and the galley proof are versions of the publication after peer review.

- The final published version features the final layout of the paper including the volume, issue and page numbers.

Link to publication

\footnotetext{
General rights rights.

- You may freely distribute the URL identifying the publication in the public portal. please follow below link for the End User Agreement:

www.umlib.nl/taverne-license

Take down policy

If you believe that this document breaches copyright please contact us at:

repository@maastrichtuniversity.nl

providing details and we will investigate your claim.
}

Copyright and moral rights for the publications made accessible in the public portal are retained by the authors and/or other copyright owners and it is a condition of accessing publications that users recognise and abide by the legal requirements associated with these

- Users may download and print one copy of any publication from the public portal for the purpose of private study or research.

- You may not further distribute the material or use it for any profit-making activity or commercial gain

If the publication is distributed under the terms of Article $25 \mathrm{fa}$ of the Dutch Copyright Act, indicated by the "Taverne" license above, 
Mechanisms involved in the control of breathing before and after birth 
Copyright (1) 1995 I.M. Kuipers

All rights reserved. No part of this publication may be reproduced, stored in a retrieval system of any nature, or transmitted in any form or by means, electronic, mechanical, photocopying, recording or otherwise, without the prior written permission of the copyholder, application for which should be addressed to the publisher.

For information: 1.M. Kuipers, Roerdomp 7, 3628 CA Kockengen, tel. (03464) 2590; after 10 october 1995 (0346) 242590.

\section{CIP-DATA KONINKLLIJKE BIBLIOTHEEK, DEN HAAG}

Kuipers, Irene Mariëtte

Mechanisms involved in the control of breathing before and after birth Thesis Maastricht - With references - With summary in Dutch.

Signal Maastricht 1995

ISBN 9075418019

Subject headings: control of breathing / fetal breathing movements / fetal lamb / birth / ECMO

Book production: Sylvia Schoenmakers

Cover painting: Hans Kuipers 


\section{Mechanisms involved in the control of breathing before and after birth}

\section{Proefschrift}

ter verkrijging van de graad van doctor aan de Rijksuniversiteit Limburg te Maastriclit, op gezag van de Rector Magnificus, Prof. Mr. M.J. Cohen, volgens het besluit van het College van Decanen, in het openbaar te verdedigen

op donderdag 20 april 1995 om 16.00 uur

door

Irene Mariëtte Kuipers 
Promotors :

Co-promotor:

Beoordelingscommissie: Prof. Dr. J. de Haan (voorzitter)

Paranimfen:
Prof: Dr. J.L.H. Evers

Prof. Dr. H.Th.M. Folgering

(Katholieke Universiteit Nijmegen)

Dr. L.L.H. Peeters

Prof. Dr. G.H.A. Visser

(Universiteit Utrecht)

Prof. Dr. C.E. Blanco

Prof. Dr. M.A. Hanson

(University College London, U.K.)

Dr. W.J. Maertzdorf

Drs. E.C.M. Kuipers

Drs. M. Ruige 


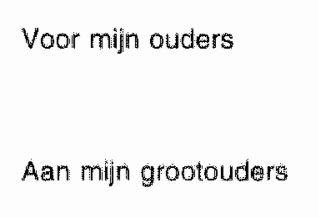




\section{Contents}

Abbreviations $\quad 6$

Chapter 1

Chapter 2

Chapter 3

Chapter 4

Chapter 5

Chapter 6

Chapter 7

Chapter 8

Chapter 9
General introduction

Rleview of the literature $\quad 13$

Materials \& methods

The effect of mild hypocapnia on breathing and behavior in unanesthetized normoxic fetal lambs

J. Appl. Physiol. 76: 1476-1480, 1994

The effect of hypercapnia and hypercapnia associated with central cooling on behavior in unanesthetized fetal lambs Submitted

The effect of maternal hypoxemia on behavior in unanesthetized normoxic and mildly hyperoxic fetal lambs J. Appl. Physiof 76: 2535-2540, 1994

Fetal breathing is not initiated after cord occlusion in the unanesthetized fetal lamb in utero

J. Dow. Physiol. 17: 233-240,1992

Initiation and maintenance of continuous breathing at birth Submitted

General discussion

Roferences

Summary

Samenvatting

Dankwoord

Curriculum vitae
7

37

105 113

133

6
7
7

49

59

71

95

141 


\section{Abbreviations}

bpm beats per minute

ECMO extracorporeal membrane oxygenation

ECOG electrocortical activity

EMG electromyographic

HV ECoG high voltage low frequency electrocortical activity

LV ECoG low woltage high frequency electrocortical activity

$\min \quad$ minutes

$\mathrm{PaCO}_{2}$ arterial $\mathrm{PCO}_{2}$

$\mathrm{PaO}_{2}$ anterial $\mathrm{PO}_{2}$ 
Chapter 1

General introduction 
In utero, breathing movements are present from early in gestation in animals and man (Connors et al. 1989, Cooke et al. 1990). This fetal breathing activity is similar to respiratory activity after birth since neurons of the respiratory centre the phrenic nerve and the diaphagm are involved (Bahoric \& Chernick 1975, loffe et al. 1992, de Vries et al. 1982). In utero breathing activity does not accomplish gas exchange because the lungs are filled with fluid and pulmonary circulation is around $15-20 \%$ of the right ventricular cardiac output (Iwamoto et al. 1987. Teitel et al. 1990). Then what is the function of fetal breathing activity? It is reported that it is necessary for lung growh and development (Fewell et al. 1981 , Liggins et al. 1981a, Liggins et al. 198 lb, Harding et al. 1993).

The presence and the regulation of fetal breathing movements has attracted the attention of several groups of investigators. First, acute animal experiments were performed, Jater chronic animal preparation without the influence of anesthetics allowed more detailed description (Barcroft 1947, Dawes et al. 1972). From those observations the knowledge of regulation of fetal breathing activity increased noticeably but of course many questions remain to be answered, e.g. a) what are the stimuli for the presence of fetal breathing movements in utero, b) which are the regulators and c) what are the changes in the regulation of breathing at birth?

Early in gestation in man breathing activity is present randomly, being a free running activity (Cooke \& Berger 1990 ). In the fetal lamb after maturation of electrocortical activity into $\mathrm{D}$ ECoG and HV ECOO breathing becomes present periodically, only during LV ECoG (Dawes et al. 1972). These obserwations generated questions on the periodic nature of breathing in utero, its central control and the changes at birth which allow the presence of continuous breathing. It would be interesting to understand fully the mechanisms involved in these changes since it can help to explain some conditions occurring after birth such as apnea of prematurity, sudden infant death syndrome and congenital central hypoventilation syndrome.

The classical challenges to respiratory function after birth, e.g. hypoxemia, hyperoxia, hypercapnia or even hypocapnia, have been studied in the fetal lamb. The expectations were to find similar responses but of course the fetus has different priorities and is in a different environment. This is clearly seem in the response to hypoxemia. In the adult, hypoxemia produces a respiratory stimulation mediated by the peripheral chemoreceptors. However, during hypoxemia fetal breathing activity is inhibited and this is due to central mechanisms (Boddy er al. 1974, Ioffe et al. 1987, Blanco et al. 1983b, Blanco et al. 1984). This response seems appropriate in utero for conservation of oxygen (Rurak \& Gruber 1983). To understand better the periodicity of breathing activity it is tempting to speculate that fetal $\mathrm{PaO}_{2}$, which is approximalely $3-4 \mathbb{K}: \mathrm{Pa}$ in late gestation, is sensed as hypoxemia and therefore exerts an inhibitory influence on breathing activity, only overridden by $\mathrm{LV}$ ECOG. However, it does not explain why breathing is present before ECoG differentiation and studies of the response range of the peripheral chemoreceptor show that they do not sense the $\mathrm{PaO}_{2}$ as hypoxia. 
Moreover, hyperoxia does not change the incidence of breathing movenients in utero (Blanco et al. 1991). This question is still debated (Hasan \& Rigaux 1992).

The rolle of $\mathrm{CO}_{2}$ in the control of breathing is better understood since the responses are similar to that seen after birth. Hypocapnia is associated with a low incidence of fetal breathing activity (Connors et al. 1988). In utero breathing activity is stimulated by hypercapnia, but it remains present periodically (Boddy et al. 1974, Janssen et al. 1982, Dawes et al. 1982, loffe et al 1987): These obserwations suggest that the level of $\mathrm{PaCO}_{2}$ plays an important role in the presence of fetal breathing activity. Furthermore, the stimulatory effect of hypercapnia can be an important mechanism in the initiation of continuous breathing at birth (Blanco et al. 1987b).

At the time of birth breathing must become present continuously, i.e. the inhibition which is present in utero must be lifted. There is a long list of factors which facilitate or generate this change, including temperature changes at birth and other afferent inputs such as towch, pain, sound and light, which may be involved in the mechanism for overcoming the central inhibition. When the umbilical cord is clamped, placental circulation is excluded, and therefore possible mediators produced by the placenta which could play a role in fetal breathing regulation, e.g. endorphins; prostaglandins and adenosine, are also excluded. It is known that in utero these mediators can influence fetal breathing activity (Kitterman et al: 1979, Kitterman et al. 1983, Grunstein et al. 1981, Bissonnette et al. 1991).

Many other factors play a role in establishing a new area for gas exchange after cord occlusion. There is a decrease in pulmonary vascular resistance, an increase in pulmonary blood flow, the lungs become expanded with gas etc. The airways are exposed to changes in $\mathrm{CO}_{2}$ which may influence upper airway receptors (Marsland et al. 1975, Haddad \& Mellins 1977, Banzett et al. 1978, Sheldon \& Green 1982). However, mechanical ventilation of the lung by itself during normoxic-normocapnia or hyperoxic-normocapnia does not initiate continuous breathing activity in utero (Blanco et al. 1988). Of the many factors which may be involved in the initiation of continuous breathing at birth, in the last few years interest has been focused on hormonal changes after cord occlusion.

The studies of the effects of hypoxemia, hypercapnia or hypocapnia in utero were previously performed by altering maternal blood gases or by reducing uterine blood flow. The exposure of the animal (in this case the ewe) to hypoxemia, hypercapnia or hypocapnia or decreasing uterine blood flow produces a stressful situation causing the release of catecholamines, ACTH and even other substances such as endorphins or prostaglandins, which are known to be respiratory modulators. This is of course a limiting and confounding factor which has to be taken into consideration when interpreting those results. Because of this problem direct access to the fetus was attempted using mechanical ventilation with continuous positive airway pressure (CPAP) or high frequency ventilation oscillation (HFO) but it was difficult to change fetal $\mathrm{PaCO}_{2}$ or $\mathrm{PaO}_{2}$ independently. Moreover many other receptors could be stimulated when 
applying mechanical ventilation or CPAP in utero. We decided to use ECMO as an alternative technique to study fetal breathing and behavioral activity. The ECMO technique is invasive for several reasons. It requires cannulation of vessels such as the jugular vein and carotid artery. The ECMO system requires an extra volume of $350 \mathrm{cc}$ in the circuit compared to the fetal blood volume of approximately $300 \mathrm{cc}$. The blood is in contact with foreign surfaces (tubing, membrane lung, bladder), therefore activation of inflammatory processes can occur. This of course could introduce confounding variables. It is therefore necessary to control for this using the same criteria as previously to judge physiological behavior, using i.e. ECoG activity, fetal breathing movements, nuchal muscle activity, rapid eye movements and cardiovascular parameters, blood gases. and $\mathrm{pH}$ (Dawes et al. 1972, Molteni et al. 1980, Clewlow et al. 1983). It is then possible to perform experiments only on fetuses whicly showed. comparable behavioral states, cardiovascular parameters and blood gases and $\mathrm{pH}$ as described in the literature. Under these conditions ECMO allowed us to control fetal blood gases $\mathrm{pH}^{\mathrm{pH}}$ and central temperature.

\section{Aims of the thesis}

\section{a) Mechanisms involved in the presence of spontaneous breathing activity in utero}

It is known that after birth breathing is closely regulated by $\mathrm{CO}_{2}$ production (Cunningham et al. 1986). The question is whether this mechanism might already be present in utero. Breathing activity is present from very early in gestation. Why this activity is present is not well understood, but it is reasonable to propose that it is dependent on metabolically produced $\mathrm{CO}_{2}$. This led to the question:

\section{Are breathing movements in utero dependent on the level of $\mathrm{PaCO}_{2}$ ?}

\section{b) Effect of cooling on $\mathrm{CO}_{2}$ sensitivity}

It is well known that fetal breathing activity is stimulated during hypercapnia but it remains periodic, since during HV ECoG breathing activity is inhibited (Boddy et al. 1974, Jansen et al. 1982, Dawes et al. 1982, Ioffe et al. 1987). The periodicity of breathing might be due to a lower sensitivity sto $\mathrm{CO}_{2}$ caused by a lower afferent input to the central nerwous system than occurs postnatally. This led to the question:

Does increased afferent input to CNS produced by central cooling change the threshold for $\mathrm{CO}_{2}$ and override the central inhibition during HV ECoG, resulting in continuous breathing? 


\section{c) Analysis of mechanisms involved in the fetal response to hypoxemia}

It is known that after birth hypoxemia stimulates breathing activity; in contrast, fetal breathing movements are inhibited during hypoxemia (Boddy et all. 1974, Bryan et al. 1986). One hypotheses is that the fall in $\mathrm{PaO}_{2}$ is sensed by a central structure, a "chemoreceptor", located in the brain stem which exerts an inhibitory response (Dawes et al. 1983). Another possibility is that $\mathrm{O}_{2}$-deficient tissues, including the placenta, release substances such as adenosine, endorphins and prostaglandins which inhibit fetal breathing activity. Previously all the experiments had been done by giving the ewe a hypoxic gas mixture to breathe or by reducing uterine blood flow making the placenta and possibly the ewe hypoxic as well as the fetus. This led to the question:

Are the inhibitory effects on fetal breathing during fetal hypoxemia an indirect effect due to release or production of mediators from the maternal side of the placenta or the ewe?

d) Mechanisms involved in the switch from periodic fetal breathing to neonatal continuous breathing activity at birth

After cord occlusion at birth the inhibition of breathing activity during HV ECoG must be overridden and breathing must become continuous. The onset of continuous breathing has been related to many factors. One of them is temperature since peripheral cooling in utero and after birth resulted in continuous breathing activity (Hamed \& Fetreiro 1973, Gluckman et al 1983). It was also proposed that the disappearance of an inhibitory substance of placental origin could play a rolle since breathing activity becomes present continuously after cord acclusion and it is inhibited again after cord release (Adamson et al. 1987, Blanco et al. 1987b). However, in those experiments fetal $\mathrm{PaCO}_{2}$ was not controlled resulting in hypercapnia. The reason for the initiation of continuous breathing after cord clamping in those experiments was thus not clear because the exclusion of a possible placental factor and hypercapnia occurred simultaneously. Thus the questions remained:

Does the exclusion of the umbilical circulation and therefore placental modulators play a role in the initiation of breathing at birth? Does a rise in $\mathrm{PaCO}_{2}$ and changes in temperature play a role during this transition? 


\section{Chapter 2}

\section{Review of the literature}

2.1 Function of fetal breathing movements

$2.2 \quad$ Initiation and development

2.3 Central control

2.3.1 Electrocortical activity

$2.4 \quad$ Metabolic control

2.4.1 Glucose

2.4.2 Carbon dioxide

2.4.2.1 Hypocapnia

2.4.2.2 Hypercapnia

2.4.3 Oxygenaition

2.4.3.1 Hypoxemia

2.4.3.2 Hyperoxia

2.4.4 Metabolic acidosis

$2.5 \quad$ Peripheral control

2.5.1 Chemoreceptors and lung afferents

$2.6 \quad$ Neuromodulators and drugs

$2.7 \quad$ Birth

2.7.1 Extra afferent input

2.7.2 Cord occlusion 


\subsection{Function of fetal breathing movements}

Fetal breathing movements are associated with phrenic nerve activity and contraction of the diaphragm (Bahoric \& Chemick 1975). It is believed that breathing activity could play a role in lung growth and development. The role of fetal breathing movements on Iung growth and development has been studied by diminishing or abolishing breathing activity by spinal cord transections (Liggins et al. 1981a, Harding et al. 1993) or phrenectomy (Alcorn et al. 1980, Fewell et al. 1981). Other investigators increased the compliance of the thorax in order to reduce the negative intrathoracic pressure generated by breathing activity (Liggins et al. 1981b). Lung growth and development were shown to be influenced by these three experimental conditions since the lungs were smaller, had lower DNA content and the distensibility of the fetal lungs decreased (Wigglesworth et al. 1979, Alcom et al. 1980, Fewell et al. 1981, Liggins et al. 1981 a, Liggins et al. 1981b, Bamford et al. 1992, Harding et al, 1993). However, Sival et al. (1992) reported no correlation between the incidence of fetall breathing movements and lung development. Bamford et al. (1992) reported that, despite a small lung, no change in collagen. elastin or DNA content was found.

Lung growth and development are also influenced by altering lung liquid volume. Drainage or ligation of the trachea for 3 to 4 weeks resulted in pulmonary hypoplasia (Alcorn et al. 1977) or an increase in pulmonary lung growth and pulmonary hyperplasia respectively (Alcom et al. 1977, Wigglesworth et al. 1979). The fall in lung liquid volume after spinal cord transection supports the wiew that fetal bresthing activity stimulates lung growth by its positive influence on lung liquid volume (Dickson \& Harding 1991, Fisk et al. 1991, Harding et al. 1993).

In summary, it seems that the presence of a normal incidence of fetal breathing movements is necessary for nomal lung growth and development. The mechanism by which fetal breathing activity influences lung growth and development is likelly to require continued and controlled release of lung liquid volume.

\subsection{Initiation and development}

Breathing movements are present in utero from early in gestation. Barcroft (1947) described a spasm of the diaphragm at the $38^{\text {th }}$ day of gestation (term 147 days) in the fetal lamb followed by the first rhythmic movements of the diaphragm at the $40^{\text {th }}$ day of gestation. The earliest chronic recordings from fetal lambs in utero were performed at approximately 50 days of gestation. Two types of diaphragmatic activity could be seen: 1) unpattemed discharge, 2) patterned bursting discharge (Cooke \& Berger 1990). Between 75 days and 120 days of gestation, movements of the diaphragm were present periodically, being mainly 
associated with nuchal and lateral rectus muscle activity, but there was no relationship with nuchal muscle activity or rapid eye movements (Clewlow et al. 1983, Ioffe et al. 1987). From 108-120 days of gestation breathing movements become more associated with rapid eye movements and increased nuchal muscle activity (Bowes et al 1981a, Clewlow et al. 1983, Joffe et al. 1987, Szeto et al 1992). Electrocontical activity is still undifferentiated and at around 116 days of gestation the frequency was low, approximately 8-16 Hz (Sxeto 1990).

In the human fetus breathing movements start to occur between the $10-12^{\text {ith }}$ week of gestation (de Vries et al: 1982).

In summary, breathing activity is present from early in gestation in animals and man. Breathing movements are present irregularly without a clear partern which suggest a nonregulated activity.

\subsection{Central control}

\subsubsection{Electrocortical activity}

In the fetal lamb the most important change in the organization of breathing and behavioral activity occurs after maturation of the forebrain. By 120-125 days of gestation electrocortical activity matures into clearly identifiable states, $\mathrm{LV} E \mathrm{ECOG}$, with a frequency of $13.30 \mathrm{~Hz}$, and HV ECoG, with a frequency of $3-9 \mathrm{~Hz}$ (Dawes et al. 1972, Clewlow et al. 1983, Szeto et al. 1985, Szeto 1990). In fetal lambs electrocortical activity alternates between $\mathrm{LV}$ ECoG and $\mathrm{HV} \mathrm{ECOG}$ after this point in gestation.

After maturation of electrocortical activity fetal breathing movements become present periodically, occurring only during $40 \%$ of the total time and only during $\mathrm{LV}$ ECoG. These fetal breathing movements are rapid and irregular, have a frequency of $0.1 .4 \mathrm{~Hz}$, an amplitude of $3-5 \mathrm{mmHg}$ and produce only small changes in lung liquid wolume $(<\mathrm{lml})$ (Harding et al. 1984).

During LV ECoG fetal breathing movements are present for $70 \%$ of the time, being associated with rapid eye movements. During LV ECOG there is an inhibition of postural muscle tone (Dawes et al. 1980, Ioffe et al. 1980) and the amplitude of spinal polysynaptic reflexes is reduced (Blanco at al. 1983b). This LV ECoG state is comparable to REM sleep after burth (Phillipson \& Bowes 1986). LV ECoG activity is associated with a significantly lower heart rate, a decrease in blood pressure, a decrease in placental blood flow and an increase in central blood flow (Clapp et al. 1980, Richardson et al. 1985, Abrams et al. 1991). Sleep state modulation of blood pressure and heart rate is independent of intact wagi and/or carotid sinus nerves (Koos \& Sameshima 1988c). The irtegularities in heart rate varability during $L$ V ECOG may be caused by the fall in intrathoracic pressures associated with respiratory movements (Dawes 1973). 
During HV ECoG breathing movements are inhibited (Dawes et al. 1983, Gluckman \& Johnston 1987, Johnston \& Glucknan 1989).

HV ECOG is associated with sustained contractions of nuchal muscles, there is spontaneous gastrocnenius activity and the magnitude of spinal monosynaptic and polysynaptic refleses are present (Dawes et al. 1972, Ruckebusch et al. 1977, Natale et al. 1981, Rigatto et al. 1982, Clewlow et all. 1983, Ioffe et al. 1987). It is not known why fetal breathing mowements are inhibited during HV ECOG and how this mechanism is overridden during LV BCoG.

Different experiments lead to the conclusion that the inhibition of fetal breathing movements during HV BCoG is of central origin (Dawes et al. 1983. Johnston Giluckman 1989). Breathing movements were dissociated from ECoG after transection at the level of the colliculi (Dawes et al. 1983) or after bilateral lesions in the lateral pons, placed stereotaxically (Gluckman \& Johnston 1987, Johnston \& Gluckman 1989). Furthermore, it has been suggested that during HV ECOG is an increase in the threshold for breathing. This was illustrated in experiments using fetall lambs with lesions in the lateral pons in the lateral parabrachial/Kolliker - Fuse region. In these fetuses breathing activity was present periodically during normocapnia, but during lactic acidosis or hypercapnia breathing activity became present continuously (Johnston \& Gluckman 1983).

So far we have described 2 sleep states, but it is also possible that wakefulness is present in utero and breathing activity could be regulated differently in that state. Fetal wakefulness was defined by the simultaneous presence of LV ECoG, rapid eye movements and neck muscle tone, being present only 5 $6 \%$ of the time (lolfe ot al. 1980 , Rigatto et al. 1982). However, observations through a Plexiglas window implanted in the flank of the ewe did not confirm the occurrence of wakefulness as defined postnatally (Rigatto et al. 1986).

In the fetal lamb during the last trimester of gestation, breathing movements may be part of the behawiond expression of $\mathrm{LV}$ ECOG since they are only present during this state. Beloavioral states are constellations of physiological and behavioral variables which are slable over time and recur repeatedly, not only in the same fetus but also in similar forms in all fetuses at a similar age (Prechtl et al. 1968). In utero HV ECoG is associated with nuchal muscle actiwity, absence of rapid eye movements and absence of breathing movements, and LV ECoG is associated with absence of nuchal muscle activity, the presence of rapid eye movements and the presence of breathing movements (Richardson et al. 1985 , Bocking \& Harding 1986).

In the human, fetal breathing mowements are also present periodically. It was reported that rapid eye movements and the presence of breathing movements were highly correlated after 27 weeks of gestation (Okat et al. 1992). Although fetal breathing movements did not contribute to the definition of fetal behavioral states, the regularity and frequency of fetal breathing movements were influenced by states (Nijhuis at al. 1982, Arabin \& Riedewald 1992, Mulder et al. 1994). Behavioral states in the human fetus were defined by fetal body movements, rapid eye movements 
and heart rate pattem. The coordination among these variables are already present around 32 weeks of gestation (Visser et al. 1987). By 38 to 40 weeks of gestation 4 distinct fetal states were present equivalent to 4 of the 5 states described in the newborn (Nijhuis et al. 1982).

In summary, electrocortical activity differentiates into two states in fetal lambs at $120-125$ days of GA. The presence of breathing activity is state dependent since breathing movements are only present during LV ECOG. During HV ECOG breathing movements are inhibited, this inhibition being of central origin. Breathing activity early in gestation seems to lack pattern, therefore it is difficult to explain its presence as a behavioral expression. In the human fetus breathing movements are periodically present but they do not contribute to the definition of fetal behavioral states since they are not limited to a definite state.

\subsection{Metabolic control}

\subsubsection{Glucose}

In the fetal lamb, as in the human, glucose is the major metabolic substrate. Fetal breathing movements are likewise influenced by circulating levels of glucose.

In the fetal lamb maternal hypoglycemia due to lack of food intake is associated with a reduction in the incidence of LV ECoG and fetal breathing movements. Infusion of glucose to fasted fetuses resulted in an increase of breathing movements to a level similar to the control values (Richardson et al. 1982).

In the human fetus, the incidence of fetal breathing movements is also influenced by glucose. Breathing movements were stimulated by hyperglycema (Natale et al. 1978, Patrick et al. 1978, Bocking et al. 1982). The stimulation of breathing activity during hyperglycemia might be explained by the oxidation of glucose to $\mathrm{CO}_{2}$ and $\mathrm{H}_{2} \mathrm{O}$. These increased concentrations of $\mathrm{CO}_{2}$ could stimulate respiratory activity (Natale et al. 1978, Bocking et al. 1982, Richardson et al. 1983). Of course until recently fetal blood gases and $\mathrm{pH}$ could only be measured in fetal sheep (Bocking al. 1982, Richardson et al. 1983). Another possibility might be that glucose increases the incidence of LV ECOG (Richardson et al. 1982)

In summary glucose levels influence fetal breatling activity.

\subsubsection{Carbon dioxide}

$\mathrm{CO}_{2}$ is an end product of metabolism and is directly related to $\mathrm{O}_{2}$ consumption under aerobic conditions. In the adult the elimination of $\mathrm{CO}_{2}$ is achieved by two mechanisms, a fast mechanism by pulmonary 
ventilation and a slow mechanism by renal excretion as bicarbonate: Therefore, there is a very close relationship of alveolat ventilation to $\mathrm{CO}_{2}$ production (Cunningham et al. 1986).

\subsubsection{Hypocapnia}

In the adult $\mathrm{CO}_{2}$ production and its oscillations play an important role in the control of breathing (Cunningham et al. 1986, Eltridge \& Millhom 1986). The maintenance of breathing activity is critically dependent on the level of $\mathrm{PaCO}_{2}$ during all stages of sleep. Mild laypocapnia $\left(\mathrm{P}_{\text {end tidal }} \mathrm{CO}_{2}<4.6 \mathrm{kPa}\right.$ ) leads to apnea, except during wakefulness when behavioral drives supervene (Datta et al. 1991, Canet et al. 1993). During the first two weeks of life the apnea threshold was $1-1.2 \mathrm{kPa}$ lower than immediately after birth which indicated resetting of this threshold (Canet et al. 1993). Thus breathing activity is modulated by $\mathrm{PaCO}_{2}$

Whether the presence of fetal breathing activity is determined by $\mathrm{PaCO}_{2}$ is still unsettled. In utero breathing activity does not provide any gas exchange since this is provided by the placenta. Therefore, it would not be logical to expect that $\mathrm{CO}_{2}$ production or $\mathrm{PaCO}_{2}$ level would control breathing activity in utero as postnatally.

To our knowledge no conclusive study of the effect of fetal hypocapnia on fetal breathing activity in the fetal lamb has been reported Boddy et al: (1974) described 2 fetal lambs that became hypocapnic and remained normoxic spontaneously due to hyperventiation of the ewe. These fetuses showed a decreased incidence (15\%) of fetal breathing activity but the duration of this effect was not reported. After administration of $3 \%$ of $\mathrm{CO}_{2}$ to the mother brearhing activity returned to its normal incidence.

In the human fetus a decrease in breathing movements has been produced during hypocapnia obtained by maternal hyperventilation in the last weeks of gestation (Marsál et al. 1979, van. Weering et al. 1979, Connors et all. 1988). These experiments were limited 10 a maximum of $15 \mathrm{~min}$ and no fetall blood gases were obtained.

In the human and sheep hypocapnia could cause a decrease in uterine blood flow (Leduc 1972, Levinson et al. 1974, Oakes et al. 1976), an increase in placental vascular resistance (Motoyama of al. 1967, Oakes el al. 1976) and a decrease in umbilical blood llow (Motoyana el al. 1967) possibly resulting in hypoxemia. Therefore, the mechanism responsible for the decrease in fetal breathing movements during hypocupnia in those experiments is not clear since hypocapnia and hypoxemia mat have occurred simultaneously (Marsal et al. 1979) and hypoxemiat is a known inhibitor of fetd breathing movements (Boddy at al. 1974).

One of the problems of fetal hypocapnia obtained by any of the methods is that it can produce a decrease in cerebral blood flow which may result in central nervous system hypoxemia and this may have been responsible for the decrease in breathing activily during hypocapnia. However, in piglets hypocapnia was associated with a significant decrease of cerebral blood flow only when $\mathrm{PaCO}_{2}$ decreased to extreme degrees 
$\left(\mathrm{PaCO}_{2}<2\right.$ kPa) and bload flow to the brain stem, thallamus and cerebellum was preserved (Hansen et al. 1984).

In summary, after birth $\mathrm{CO}_{2}$ level infuences breathing activity but in utero this is less clear. Hypocapnit expenments were performed by maternal hyperventiation which might have resulted in hypoxemia due to a decrease of uterine blood flow, an increase in placental vascular resistance and a decrease of umbilical blood flow. Furthernore, these experiments were limited in duration. Thus whether the incidence of fetal breathing movements are influenced by hypocapnia remains unclear.

\subsubsection{Hypercapnia}

Hypercapnia is a well known respiratory stimulant which produces its effect at peripheral and central chemoreceptors. The effects are expressed. as a stimulation of ventilation which is clearly directed io decrease $\mathrm{PaCO}_{2}$. What is the situation in utero?

From 0.5 of gestation in the fetal lamb hypercapnia causes a change from irregular tonic to regular phasic activity in diaphragmatic $\mathrm{EMG}$ activity already (Ioffe et al. 1987). In the human fetus hypercapnia stimullates breathing activity from 24 weeks" of gestation (Connors et al. 1989).

During hypercapnia in fetal lambs, after maturation of electrocontical activity, there is an increase in incidence of breathing activity and it becomes deeper and more regular (Boddy et al. 1974, Chapman et al. 1980, Dawes et al. 1982). Hypercapnia is not sufficient to produce continuous breathing since breathing activity remains associated with LV ECOG and is inhibited during HV ECoG (Jansen et al. 1982, Rigatio et al. 1988). Therefore, the increase in incidence of breathing activity appears to be due to a change in behavioral activity since there is a significant increase in incidence of $\mathrm{LV} \mathrm{ECoG}$, the state in which fetall breathing activity occurs (Boddy et al. 1974). An increase in breathing activity in hypercapnia is also described in human fetuses (Ritchie \& Lahkani 1980, Connors et al. 1988, Connors et al. 1989). However, in the human fetus there is no change in behavioral activity during hypercapniat, which suggests that in the human fetus hypercapnia stimulates the respiratory centre directly rather than via a change in behavioral activity (O'Grady et al. 1983).

The mechanisms involved in the stimulation of breathing during hypercapnia are not known. In utero peripheral chemoreceptors are active and responsive to natural stimuli (Blanco et al. 1984). Injection of $\mathrm{CO}_{2}$ saturated salline towards the carotid chemoreceptors resulted in an increase in their firing activity. However, after carotid denervation and vagal section the incidence of fetal breathing movements still increased significantly during hypercapnia (Koos \& Sameshima 1988c). Therefore, it has been suggested that the response is mediated by the central chenoreceptors (Dawes al 1982, Jansen et al. 1982, Koos er al. $1988 \mathrm{c})$. An elevation of $\mathrm{PaCO}_{2}$ alone is not sufficient to produce 
breathing in fetal lambs during HV ECoG (Dawes et al. 1982, Jansen et al. 1982, Rigatto et al. 1988). This suggests that during HV BCoCir there is a higher threshold for stimulation of breathing, due to an inhibition which is lifted during LV ECoG. The response to hypercapnia was similar in intact fetal lambs and in caudal brain stem sectioned fetal lambs. In rostral brain stem sectioned fetal lambs the response to $\mathrm{CO}_{2}$ was greater than in intact lambs. This suggests that a degree of control by higher centres operates normally in intact animals (Dawes al 1983). This was further studied in fetal lambs with lesions in the rostral lateral pons. In those animals breathing activity remained associated with LV ECoG during normocapnia. Hypercapnia produced an incresse in incidence of breathing, and breathing activity became continuous in 4 fetal lambs and almost continuous in another 3 fetall Jambs (Johnston \& Gluckman 1989).

These results suggest that there is a central regulation of the $\mathrm{CO}_{2}$ sensitivity in utero which is dependent on a small area in the rostral lateral pons (nucleus or pathway).

In utero $\mathrm{CO}_{2}$ is a potent stimulus of breathing activity which is convenient since hypercapnia plays an important role in the initiation of continuous breathing at birth (Blanco et all. 1987b). At the time of birth there is an increase in afferent input from cooling, touch, pain, sound and light and these stimuli can generate or facilitate the drive to breathe (Condorelli \& Scarpelli 1975, Scarpelli et al. 1977, Gluckman et al. 1983). Indirect evidence supporting this was reported by Moss \& Scarpelli since the threshold for $\mathrm{CO}_{2}$ was lowered in acute experiments in fetal lambs by sciatic nerve stimulation or cooling (Moss \& Scarpelli 1979, Moss et al. 1983). Furthermore, at the time of birth cold could override the negative effects of hypocapnia (Blanco et al. 1987b). This could lead to the idea that extra afferent input might change the sensitivity for $\mathrm{CO}_{2}$ resulting in the initiation of continuous breathing at birth.

In most experiments fetal hypercapnia was obtained by producing maternal hypercapnia. Therefore, the fetal responses were not independent of maternal influences. Matemal hypercaphia can result from satressful situation which can cause an increase in fatal plasma epinephrine (Faucher et al. 1991), maternal and fetal cardiovascular changes, such as hypertension and tachycardia, consistent with sympathetic activation (Walker et all. 1976),

In summary, hypercapnia stimulates fetal breathing activity but it remains present periodically. It may be speculated that extra afferent input might change the sensitivity to $\mathrm{CO}_{2}$, contributing to the initiation of continuous breathing at birth. So far fetal hypercapnia experiments have been obtained by maternal hypercapnia which limits interpretation and introduces confounding variables. 


\subsubsection{Oxygenation}

Fetal $\mathrm{PaO}_{2}$ is low (approximately $3 \mathrm{kPa}$ ) but the oxygen supply is twice the walue necessary to maintain normal $\mathrm{O}_{2}$ consumption with aerobic metabolism (Wilkening \& Meschia 1983). This level of oxygenation allows the presence of nomal behavior, fetal growth and development. Nevertheless, fetal breathing movements are present periodically and hypoxemia exerts a centrally-mediated inhibitory influence (Dawes et al. 1983). It has been suggested that fetal normoxemia might be sensed as hypoxemia resulting in an inhibition which might cause periodicity of fetal breathing. The presence of fetal breathing movements during LV ECOG can be seen as a lifting of the inhibition by this state. Furthermore, the fact that at birth breathing becomes continuous through all states may be interpreted as a lifting of this tonic inhibitory influence by the increase in $\mathrm{PaO}_{2}$ which follows birth. The question is whether this mechanism would explain inltiation of breathing after cord occlusion at birth. It is highly unlikely that $\mathrm{PaO}_{2}$ plays an important role in the initiation of breathing actiwity at birth since $\mathrm{PaO}_{2}$ at birth is at fetal level or even lower (Berger et al. 1990) but oxygenation after birth is essential. for the maintenance of breathing.

In summary, oxygenation might play a role in determining the periodicity of fetal breathing activity.

\subsubsection{Hypoxemia}

After birth, hypoxemia produces a stimulatory effect, there being an increase in ventilation, arousal and persistence of spinal reflexes ( $\mathbb{B}$ lanco et al. 1983a, Cunningham et al. 1986, Phillipson \& Bowes 1986). This response seems to be mostly mediated by the peripheral chemoreceptors since after chemodenervation the effect of hypoxemia tends to be ithibitory.

In utero, the response to hypoxemin is different, as it causes inhibition. This inhibitory fetal response is present within 5 min of exposing the ewe to severe hypoxemia $\left(9 \% \mathrm{O}_{2}, 3 \% \mathrm{CO}_{2}\right.$ and $\left.88 \% \mathrm{~N}_{2}\right)$ (Boddy et al. 1974, Koos et al. 1987a). This overall inhibition of fetal activity is represented by a decrease of limb movements, nuchal muscle activity (Natale et al. 1981, Bocking \& Harding 1986, Martin et al. 1987, Woudstra et al. 1990) "rapid eye movements (Boddy et al. 1974, Natale et al. 1981, Bocking \& Harding 1986, Koos et al. 1987a), fetal swallowing (Sherman et a. 1991) and a reduction of the magnitude of hind-limb reflexes (Blanco et al. 1983b). The effect of hypoxemia on ECoG activity is controversial as the incidence of LV ECoG activity is reported to decrease (Boddy et al. 1974, Natale et al. 1981, Bocking \& Harding 1986) or remain unchanged (Adamson et al. 1984, Koos et al. 1987a, Bocking et al. 1988). The number of switches from HV ECoG to LV ECoG activity is reported to increase (Boddy et al. 1974, Clewlow et 
al. 1983, Koos et al. 1987a) or to remain unchanged (Adamson et al. 1984, Bocking \& Harding 1986). Therefore, during hypoxemua $\mathrm{ECOG}$ activity may or may not change. However, the usual associations within states are missing, for instance during HV ECoG there is an inhibition of reflexes and of nuchal muscle activity (Blanco et al 1983b, Woudstra et al. 1990). Furthermore, LV ECoG state is also not complete since there are no breathing mowements and rapid eye movements present.

The mechanisms regulating fetal breathing movements, rapid eye movements or spinal reflexes seem to be different since during a prolonged hypoxemia period (48 hours) rapid eye movements were present at the end of the period but breathing movements were inhibited (Bocking et al. 1988). Also the mechanisms involved in the inhibition of spinal reflexes and breathing movements are different. In fetal lambs transected at the level of the colliculli hypoxemia stimulated breathing movements but spinal reflexes were inhibited (Blanco et al. 1983b).

The decrease of fetal breathing movements and muscle activity can be considered a logical fetal response since fetal breathing movements and muscle movements are associated with increased oxygen consumption (Rurak \& Gruber 1983). It is reported that oxygen consumption is diminished during hypoxemia (Parer 1980).

This inhibitory response exists already at 0.5 of gestation (Clewlow et al. 1983, loffe et al. 1987). However, fetal breathing activity is less affected in early gestation than in late gestation (Clewlow et al. 1983, Bocking \& Harding 1986).

Several methods have been used to study the effect of hypoxemia on fetal activity. The two most common methods for reducing fetal $\mathrm{PaO}_{2}$ are occlusion of the maternal common internal iliac artery or maternal hypoxia induced by making the ewe breathe a hypoxic gas mixture. Other possibilities for inducing fetal hypoxemia are decreasing $\mathrm{O}_{2}$ carrying capacity of the fetal blood with a decrease in viscosity (fetal anemia), by decreasing $\mathrm{O}_{2}$ carrying capacity of the fetal blood without a decrease in viscosity (fetal methemoglobinemia) or by changing oxygen affinity (carboxyhemoglobinemia) (Koos et al. 1987b, Koos et al. 1988b, Koos t. al. 1990a). Hypoxemia obtained by any of these methods resullts in a decrease of fetal breathing mowements and rapid eye movements (Boddy et al. 1974, Bocking \& Harding 1986, Bocking et al. 1988, Koos et al. 1988 a, Koos et al. 1990a). Since anemia resulted in decteased oxygen content without changing $\mathrm{PaO}_{2}$, this might imply that inhibition is triggered by low tissue oxygenation rather than actual values of $\mathrm{PaO}_{2}$ (Koos et al. 1987b).

The inhibitory effects on fetal breathing movements produced by hypoxemia or acute anemia were transient since the incidence of breathing actiwity retumed to control within $14-16$ hours after initiation of hypoxemia or hypocapnic hypoxemia (Bocking et al. 1988, Koos et al. 1988a) or within 2-3 hours during anemia (Bissonnette \& Hohimer 1987, Matsuda et al. 1992). The mechanisms involved in this adaptation are unclear (Hohimer \& Bissonnette 1991).

The mechanisms involved in the fetal inhibitory response to hypoxemia are not yet clear. The inhibition of fetal breathing movements during 
hypoxemia seemed not to be due to lack of peripheral chemoreceptor input since carotid and aortic chemoreceptor afferent discharge activity increased as $\mathrm{PaO}_{2}$ decreased (Blanco et all. 1984). Therefore, the lack of an stimulatory esponse is not due to diminished input but to an increased threshold for responding to this activity.

Evidence against a non-specific direct hypoxic depression came from work of Barcrof (1947) and Dawes et al. (1983) where the inhibitory effect of hypoxemia on breathing movements in fetal sheep was abolished by brain stem transection at the level of the colliculi (at the pontine-midbrain junction) (Dawes et al. 1983). Gluckman and Johnston reported similar observations after producing lesions within the pons in the region of the lateral parabrachial and Kolliker-Fuse nuclei close to the trigeminal motor nuclei (Gluckman \&ohnston 1987, Johnston \& Gluckman 1989 ). In these preparations breathing activity was present periodically but was stimulated during hypoxemia. The hypothesis that an area in the pons could function as a sensor for hypoxemia was further investigated in kittens and newborn lambs. Stimulation in the rostral ventral pons resulted in an imhibition of breathing activity (Coles et al. 1989, Noble \& Smith 1991) and the discharge frequencies of neurones in this area increased significantly during hypoxemia (Noble \& Williams 1989). This supports the view that the response to hypoxemia is mediated or integrated within the pons.

It is known that hypoxia reduces mitochondrial ATP production and this could be the mechanism by which hypoxemia is sensed by the putative 'pontine chemoreceptor' resulting in inhibition of fetal breathing activity. Evidence for this was suggested by experiments using Oligonycin B (inhibitor of mitochondrial ATPase) where the incidence of breathing movements was decreased without changes in ECoG activity or rapid eye movements (Koos et al. 1986). The experiments described tend to support the idea that tissue $\mathrm{PO}_{2}$ or oxygenation is the stimulus to trigger this fetal inhibition. It is possible that other mechanisms or stimuli are involved in this response still using this pontine structure or pathway as a component in the response.

During hypoxemia, $\mathrm{O}_{2}$ deficient tissues, including the placenta, are likely to release adenosine endorphins, prostaglandins etc. These substances can influence fetal breathing mowements, other muscle activities and $\mathrm{ECOC}$ activity.

It was suggested that adenosine played a role in modulating fetal breathing activity during normoxia and hypoxemia in fetal sheep (Bissonnette et al. 1990, Koos et al. 1990b). During hypoxemia, due to a decrease in oxidative phosphorylation, adenosine increased centrally or peripherally (Koos et al. 1990b, Koos \& Doany 1991). It is known that placental tissue produces adenosine during hypoxema (Slegel et al. 1988). The effects of adenosine are very similar to the effects of hypoxemia, both before and after brain stem transection in fetal lambs (Dawes et al. 1983, Koos et al. 1992). The intravenous infusion of an adenosine analogue in intact fetal lambs produced a temporary reduction of breathing and rapid eye movements but after brain stem transection breathing activity increased. Therefore, it was suggested that adenosine 
played an important role in producing the hypoxemic response and that it could be inwolved in the transmission of this inhibition locally (Koos et al. 1990b, Koos \& Doany 1991). Adenosine antagonists, e.g. theophylline, stimulated breathing movements during normoxia (Bissonnette et al. 1990 , Bissonnette el al. 1991) and prevented the inhibition during bypoxemin (Koos et al. 1990b).

Endorphins are elevated during hypoxemia in fetal lambs (Wardlaw et al. 1981). In these experiments, in which the ewe was breathing a hypoxic gas mixture, maternal beta-endorphin immunoactivity were not changed which indicated that the increase in fetal beta-endorphin was not of maternal origin. This rise in fetal endorphins might be due to an increase in the secretion of endorphins from the fetal pituitary, the placenta or to decreased degradation of endorphins. It might be speculated that the increased levels of endorphins play a role in the inbibition of felal breathing activity. It was also reported that endorphins were involved in the pathogenesis of apnea in the newborn (Chernick at al. 1981, Grunstein et al. 1981. McQueen 1983, Santiago \& Edelman 1985).

$\mathrm{PGE}_{2}$ is known to inhibit fetal breathing movements (Kitterman et al. 1983). However, during hypoxemia serum $\mathrm{PGE}_{2}$ levels were only significantly increased after 4 hours of hypoxemia and remained elevated for 24 hours (Akagi et al. 1990a, Akagi \& Challis 1990b, Sue-Tang et al. 1990). Therefore, a role of $\mathrm{PGE}_{2}$ as mediator for the inhibitory response appears unlikely since during hypoxemia breathing movements are inhibited within 5 min and return to baseline within 16 hours (Boddy et al. 1974, Bocking et al. 1988, Koos et al. 1988a).

During hypoxemia there is a significant increase in norepinephrine and epinephrine (Lewis et al. 1982). However, the effect of catecholamines on breathing activity remains unsettled since the incidence of breathing activity decreased, increased or did not change during nomoxia (Murata et al. 1981, Cohen et al. 1982 , Jansen et al. 1986). During hypoxemia infusion of a beta-agonist resulted in a decrease in breathing activity (Jansen et al. 1986). Infusion of an alpha-antagonist resulted in the presence of breathing activity even during hypoxemia (Banford \& Dawes 1990, Guussani et al. 1992). It has been suggested that alpha-adrenergic mechnisms have an inhibitory influence on fetal breathing movernents even during normoxia and hypoxia.

After infusion of modulators or drugs (660 ug morphind, meclofenanate, corticotrophin releasing hormone, thyrotropin releasing homone, 5-HTP, GABA-antagonist picrotoxin) breathing activity may either increase or changed from intermittent to continuous brealhing activity. The tonic inhibition or threshold during HV ECoG can be lifted by different mechanisms, however, the inhibitory effects of hypoxemia are still present (Guilligan et al. 1981, Johnston \& Gluckman 1983, Koos 1985, Bennet et al. 1988, Bennet et al. 1990b).

In summary, the fetal response to hypoxemia before birth is clearly inhibitory in contrast to that after birth. Stimuli for this response are a decrease in tissue oxygenation by low $\mathrm{PaO}_{2}$ or oxygen content. The mechanisms involved in this response seem to be integrated or mediated 
in the brain stem. Neurotransmitters and hormonal changes could play a role but this is not very well understood.

\subsubsection{Hyperoxia}

In the adult hyperoxia can result in transient changes in breathing activity within seconds by silencing the spontaneous activity of peripheral chemoreceptors. This relatively small effect of hyperoxia on the control of breathing appears to be a natural consequence of the only minor impact of hyperoxia on tissue oxygenation (Dejowrs 1962). The situation in the fetus is different since the fetus is chronically exposed to a low $\mathrm{PaO}_{2}$ in comparison to postnatal values. This physiological low $\mathrm{PaO}_{2}$ could be sensed as hypoxemia and exert a central tonic inhibition, only lifted during LV ECoG. Therefore, it could be attractive to think that this inhibition could be lifted at birth allowing continuous breathing to occur.

In chronically instrumented fetal lambs, raising fetal $\mathrm{PaO}_{2}$ to neonatal levels (8-12 $\mathrm{kPa}$ ) using ECMO failed to induce continuous breathing activity (Blanco et al. 1991) nor did continuous breathing occur, when fetal $\mathrm{PaO}_{2}$ was raised by expanding the lungs using mechanical ventilation (Blanco et al. 1987 ). These results were consistent with the results of Hasan et al. (1991) who reported no change in breathing activity in fetuses with a gestational age $<134$ days. However, in older fetuses it was reported that breathing activity became continuous in one of the six fetuses of $135 \pm 1$ days gestation at a $\mathrm{PaO}_{2}$ of $8.8 \mathrm{kPa}$ and in four of the six fetuses of $138 \pm 1$ days of gestation. In these latter fetuses the $\mathrm{PaO}_{2}$ increased to a mean of $32 \mathrm{kPa}$. In a later study Hasan et al. (1992) reported that fetal breathing activity became present continuously and was associated with arousal (defined by LV ECoG and the presence of nuchal muscle activity and rapid eye movements). In seven of the ten fetuses (135-142 days of gestation) $\mathrm{PaO}_{2}$ was approximately $15 \mathrm{kPa}$. In these experiments hyperoxia was obtained using CPAP to a pressure of up to $40 \mathrm{~cm} \mathrm{H}_{2} \mathrm{O}$ (Hasan et al. 1991, Hasan et al. 1992). The effect of the lung distension on fetal cardiovascular and fetoplacental blood flow induced by these levels of CPAP is not known. Towards term the effect of the increase in intrathoracic pressure induced by CPAP would be more profound in the relatively mature fetuses due to a rise in lung compliance. Contounding variables induced by these levels of CPAP cannot be excluded. Baier et al. (1990, 1992a, 1992b) reported that during hyperoxia breathing activity also became present continuously in association with cord clamping, but again the levels of fetal $\mathrm{PaO}_{2}$ were unphysiologically high and occurred most of the time with a significant increase of $\mathrm{PaCO}_{2}$ and decrease in $\mathrm{pH}$. Other mechanisms could have been involved in producing continuous breathing activity, e.g. a significant decrease in cerebral blood flow occurred when fetal $\mathrm{PaO}_{2}$ was between $10-25 \mathrm{kPa}$ (Blsnco et al. 1988) resulting in a rise in $\mathrm{PaCO}_{2}$ in the environment of the medullary chemoreceptors. So far, the presence of continuous breathing activity in utero during hyperoxia was mainly associated with an unphysiological increase of $\mathrm{PaO}_{2}$ an increase in $\mathrm{PaCO}_{2}$ and a decrease in 
pH. Only in fetuses older than 134 days gestation did breathing become present continuonisly occasionally, with a physiological $\mathrm{PaO}_{2}$ but with lung distended at a CPAP around $20-40 \mathrm{~cm} \mathrm{H}_{2} \mathrm{O}$. These experiments show that fetal maturity may play a role, but clearly they also raise many other questions.

Maternal hyperoxia can be obtained by the ewe breathing a hyperoxic gas mixture (Khazin et al. 1971, Boddy at al. 1974, Towell et al. 1984). The increase in fetal $\mathrm{PaO}_{2}$ is not proportional to the morease in matemal $\mathrm{PaO}_{2}$ (Khazin et al. 1971, Boddy et al: 1974). Hyperbaric oxygenation (Tiktinsky et al. 1992), mechanical ventilation in utero (Blanco et al. 1987a) or connection to an ECMO system (Blanco et al. 1991) are other possibilities for raising fetal $\mathrm{PaO}_{2}$. During hyperbaric oxygenation $\mathrm{PaCO}_{2}$ increases significantly which has a stimulatory effect on breathing activity. During mechanical ventilation, the lungs are distended and breathing activity may have been influenced by upper airway $\mathrm{CO}_{2}$ sensitive receptors since $\mathrm{CO}_{2}$ passes the airway (Marsland et al. 1975, Haddad \& Mellins 1977, Banzett et al. 1978, Sheldon \& Green 1982).

In summary, in fetuses up to 134 days of gestation, breathing activity does not become present continuously during hyperoxia. In fetuses older than 135 days of gestation the results are still controversial since frequently $\mathrm{PaO}_{2}$ was unphysiologically high.

\subsubsection{Metabolic acidosis}

It is well known that in the adult central metabolic acidosis stimulates breathing activity (Pappenheimer et al. 1965). In utero the effect of metabolic acidosis was studied by infusing $\mathrm{NH}_{4} \mathrm{Cl}, \mathrm{HCl}$ and lactic acid i.v. or by infusing $\mathrm{HCl}$, mock $\mathrm{CSF}$ with low $\left[\mathrm{HCO}_{3}{ }^{\circ}\right]$ and low $\mathrm{pH}$ into the ventriculocisternal system.

Metabolic acidemia produced by peripheral infusion of $\mathrm{NH}_{4} \mathrm{Cl}$ or $\mathrm{HCl}$ resulted in an increase in incidence and depth of breathing movements but: breathing seldom became continuous (Molteni et al. 1980, Hohimer \& Bissonnette 1981, Koos 1985). These results contrast with those of Johnston and Gluckman (1989) who reported that peripheral infusion of lactic acid resulted in a decrease of breathing activity during $\mathrm{LVECOO}$.

The effect of central infusion of artificial $\mathrm{CSF}$ with low $\left[\mathrm{HCO}_{3}{ }^{-}\right]$ content resulted in an increase in incidence and amplitude of fetal breathing movements and breathing activity became present continuously (Hohimer et al. 1983, Koos 1985, Walker \& Davies 1986).

In fetuses with lesions in the rostral lateral pons peripheral infusion of lactic acid resulted in breathing activity both during $L V E C O G$ and HV ECoG (Johnston \& Gluckman 1989).

In summary, metabolic acidosis stimulates breathing activity. Continuous breathing activity occurred in only a few fetuses alter peripheral infusion of $\mathrm{NH}_{4} \mathrm{Cl}$. Central metabolic acidosis obtained by $1 \mathrm{ow}\left[\mathrm{HCO}_{3}\right]$ overrides the inhibition of HV ECOG and allows continuous breathing activity in 
wtero. It is belleved that the stumulation of this breathing activity is mediated by central chemoreceptors in the medulla.

\subsection{Peripheral control}

After birth peripheral control is an important component in the control of breathing. These peripheral mechanisms are manly directed to provide information from the periphery to the CNS about the environment and the function of the lung. In utero oxygenation is provided by the placenta. Therefore, the lungs do not function as a gas exchange area and the fetus is protected from the enwironment. This provide some circumstantial evidence that peripheral information to the fetal brain is not of importance for the control of this "fetal respinatory activity".

\subsection{Chemoreceptors and lung afferents}

At birth, the carotid body chemoreceptors are not essential for the establishment of effective thythmic breathing (Jansen et a.. 1981). In utero, fetal breathing is clearly modulated by central mechanisms as suggested by the changes with ECoG state. Futhermore, chemoreceptors and vagal afferents seemed to be of little infiuence since vagotomy and carotid chemodenervation did not change the characteristics of fetal. breathing activity (Dawes et al. 1972, Boddy et al. 1974). In the fetal lamb vagotomy did not modify the size, frequency or incidence of rapid imegular breathing movements (Boddy et al. 1974) and carotid sinus denervation did not alter the incidence or characteristics of fetal breathing movements (Jansen et al. 1981, Moore et al. 1989). Murai et al. (1985) reported a decrease in incidence and amplitude of breathing movements after bilateral section of both vagus and carotid sinus nerves, although the incidence was normal 5 days after of surgery. This reported decrease might had been a result of surgery. Physiological denervation of the peripheral chemoreceptors by hyperoxia did not change the incidence of fetal breathing activity (Blanco et al. 1991). However, carotid chemoreceptors are active in utero (Blanco et al. 1984, Johnston at al. 1994). It is known that carotid chemoreceptors do have a spontaneous activity and redet to an increase in $\mathrm{CO}_{2}$ and a decrease in $\mathrm{O}_{2}$. Therefore, there must be a central mechanism which will increase the threshold for receiving and processing this information. Even though pulmonary afferents seem not to be actively involved in the regulation of breathing actiwity in utero, they can be involved when the fetal lung is inflated or deflated.

Irritant receptors are located throughout the epithelium of the upper and lower arway. After birth these receptors ane stimulated by smoke, irtitant gases or fluid and cause changes in frequency or depth of breathing. The afferent activity of these receptors is mediated by nyelinated fibres of the nervus wagus. The response of the irrilant receptors can be elicited after 35 weeks gestation in the human (Fleming 
et al. 1978). In utero the intant receptors are comtinuously exposed to lung fluid. The function (if any) of these receptors during fetal life is not known.

After birth mixed wenous chemoreceptors and intrapulmonary chemoreceptors stimulate breathing activity (Sheldon \& Green 1982). In utero pulmonary blood flow is only $20 \%$ of the right ventriculat output. There is no data on the function of mixed venous and intrapulmonary chemoreceptors during fetal life. However, since vagotomy and denervation did not affect fetal breathing activity in utero, their function does not seem to be of importance. At birth there is a significant increase in pulmonary blood flow and the amount of $\mathrm{CO}_{2}$ delivered to the lungs increases. If the $\mathrm{CO}_{2}$ receptors are active they may play a powerful role in the stimulation of breathing activity at birth.

In summary, peripheral chemoreceptors (aortic and carotid), the vagus and its afferents seems to play a minor role in the regulation of spontaneous fetal breathing activity.

\subsection{Neuromodulators and drugs}

Many artempts have been made to understand the control mechanisms of breathing activity using pharmacological interwentions.

Neurotransmitters e.g. norepinephrine, epinephrine, serotonin, act quickly over short synaptic distances. Neurohormones act at a distance from their place of release and cause more long lasting effects (e.g. adenosine, opioids " prostaglandins).

During HV ECoG plasma norepinephrine and epinephrine levels were higher than during LV ECoG (Reid et al. 1990). The effect of infusion of norepinephrine and epinephrine is to stimulate breathing activity (Bamford et al. 1986, Jansen et al. 1986, Moore at al. 1994). Isoproterenol stimulated breathing activity, but this might be related to the increase in brain metabolism (Murata et al. 1981. Jansen et al. 1986). Drugs which increased central catecholamines (DMI; desipramine, 6-OHDA; 6-hydroxydopamine) induced an increase of breathing activity associated with an increase in LV ECoG (Joseph \& Walker 1990). At birth norepinephrine tumover increased significantly after $1-2$ hours breathing air (Lagercrantz al al 1992). A transient increase in catechollamines at the time of birth may thus contribute to the initiation of breathing activity.

Pilocarpine (cholinergic agonist) stimulated breathing activity in normal (Brown et al. 1981, Jansen et al. 1983, Hinman \& Szeto 1988, Hanson et al. 1988) and chemodenervated fetal lambs in utero (Hanson et a. 1988). Pilocarpine appears to act above the level of the colliculi causing a change of $\mathrm{BCOG}$ activity to $\mathrm{LV} \mathrm{ECOG}$, and after infusing cholinergic antagonists ECoG activity switched into HV ECoG. Since pilocarpine also stimulated fetal breathing activity in brain stern transected. fetuses, the medulla or pons appears to be involwed (Hanson et al. 1988). Atropine blocked the pilocarpine induced breathing actiwity which 
suggested that a cholinergic link may be involved in the regulation of fetal breathing activity (Hanson et al. 1988, Hinman \& Szeto 1988).

Doxapram stimulated breathing activity in $\mathrm{LV} E \mathrm{ECOG}$. The site of action must be the pons or the medulla since the response was unaffected by brain stem transection and chemodenervation (Jansen et al. 1983 , Bamford et al. 1986).

Peripheral infugion of 5-hydroxytryptophan (converted to serotonin) induced continuous breathing activity in fetuses $>127$ days of gestation. Furthermore, breathing activity was more regular and there was an uncrease in amplitude (Quilligan et al. 1981): Consistent with those results are the results of Fletcher et al. (1988) who infused 5-hydroxytryptophan together with antagonists of 5-hydroxytryptamine and blocked the stimulation of breathing activity.

During thyrotrophin releasing hormone (TRH) infusions breathing activity became continuous (Bennet et al. 1988 ), TRH positive fibres were located throughout the medulla pons region of the rat brain (McCown et al. 1986). At birth, there was a rise of TSH which was dependent on the fall in environmental temperature experienced at birth (Sack et al. 1976). Since TRH was effective at very low doses it was speculated that TRH was involved in the switch from fetal to postnatal patterns of respiration at birth (Bennet et al. 1988).

Breathing activity became present continuously during infusions of corticotrophin releasing factor (CRF) and both frequency and amplitude increased (Bennet et al. 1990b). CRF antagonists inhibited fetal breathing activity during LV ECoG in normoxic fetal lambs, suggesting that CRF may have a tonic role in the generation of fetal breathing movements.

In the human, maternal consumption of caffeinated and decaffeinated coffee increased fetal breathing activity after 3 hours. Caffeine crossed the placenta rapidly. The 3-hour delay suggested that another mechanism played a role e.g. an increase in plasma glucose. Coffee raised slightly the maternal glucose level and the incidence and frequency of fetal breathing movements were significantly increased in response to glucose injections (Bocking et al. 1982). This increase in breathing activity was most probably due to increased concentrations of $\mathrm{CO}_{2}$ which influenced the respiratory centre in the medulla of the fetal brain since glucose was oxidized aerobically within the fetus and metabolized to $\mathrm{CO}_{2}$ and $\mathrm{H}_{2} \mathrm{O}$.

Theophylline, an adenosine antagonist, stimulated breathing movements during normoxia (Bissonnette et al. 1990, Bissonnette at al. 1991) and prevented the inhibition during hypoxemia by blocking the adenosine $\mathrm{R}_{1}$ receptors (Hedner et al. 1982).

A biphasic breathing response (apnea followed by significantly deeper breathing movements) occurred when morphine was infused and this response was dose related. The apnea was correlated with the highest plasma concentrations and the onset of continuous breathing coincided with the lowest plasma concentration of morphine (Hasan et al. 1988). After brain stem section the period of apnea was significantly longer and continuous breathing activity did not occur which suggested that apnea was induced by inhibiting neurones directly, and continuous breathing 
activity by disinhibition of more rostral neurones which inhibited the respiratory neurones (Hasan et al. 1990).

Fetal breathing activity was inhibited when the fetus was exposed to pentobarbitone and there was also a decrease in LV ECOG (Boddy et al. 1976).

$P G E_{2}$ is a well-known modulator of breathing. The fetal membranes and cotelydons are one of the major sources of circulating prostaglandins in the fetus (Olson et al. 1986). In newborn lambs breathing activity decreased during peripheral infusion of $\mathrm{PGE}_{2}$ and increased during meclofenamate infusion (Guerra at al. 1988, Guerra et al. 1989). Several groups reported that administration of prostaglandin or prostaglandin synthetase inhibitors (indomethacin, meclofenanate) modulated fetal breathing movements (Kitterman et al. 1979, Kitterman et al. 1983, Wallen et al. 1986, Patrick et al. 1987, Hallak et al. 1992). Fetal breathing movements were even present in HV ECoG after infusion of indomethacin or meclofenamate (Kitterman et al. 1979, Wallen et al. 1986), which suggests that endogenous production of prostaglandins inhibits breathing activity in utero during HV ECOG and might be one of the mechanisms controlling breathing activity in utero. Prostaglandins act centrally in the lower pons or medulla to modulate fetal breathing movements (Jansen et all. 1984, Koos 1985). The mechanism by which they act might be on the respiratory nuclei, via the efferents or at the central chemoreceptors. In neonatal lambs the effects of $\mathrm{PGE}_{2}$ were due to reduction of peripheral chemoreceptor discharge (Bennet \& Hanson 1990a).

Prostaglandins do not appear to have effects on ECoG activity, rapid eye movements, blood pressure, heart rate or the excitability of the neural pathways of the cranial (digastric) or spinal (flexor) reflexes (Walker 1990).

Ventriculocisternal or i.v.infusion of meclofenamate resulted in breathing activity even during HV ECOG. These breathing movements became more regular during $\mathrm{HV}$ ECOG (as postnatally) than during $\mathrm{LV}$ ECOG. The incidence of breathing activity remained high during 60 hours of infusion (Wallen et al. 1988). This long-term effect of meclofenamate is opposite to the transient inital effect of indomethacin since infusion of indomethacin into the maternal femoral vein for 70 hours induced an increase in incidence and amplitude of breathing activity with a peak at 8 to 10 hours. However, but the effect was no longer present after 20 hours despite ongoing indomethacin unfusion for a totall period of 70 hours (Patrick et al. 1987). The human fetus showed al significant increase of breathing activity, but there was also no change in fetal movements (Hallak et al. 1992). A possible mechanism by which indomethacin increases the incidence of fetal respiratory activity might be by stimulating central chemoreceptors by producing cerebral aucidosis (Hohimer et al. 1983, 1985). Altering glucose concentrations (Murai at all. 1984), changes in arterial blood gas tensions (Kitterman 1979, Murai et al. 1984 Koos 1985) and pH (Jansen et al. 1984, Hohimer ot al. 1985, Wallen et al. 1986) or an increase in the incidence of LV ECOG activity (Kitterman et al. 1979) did not occur during infusion of prostaglandin 
inhibitors and did not play a role in the increase of breathing actiwity even during $\mathrm{HV}$ ECOG. Meclofenamate is known to uncouple phosphorylation resulting in an increase in $\mathrm{CO}_{2}$ production which could stimulate breathing activity (Whitehouse \& Haslam 1962).

During the days before delivery breathing activity decreases significantly, and there is a significant increase of $\mathrm{PGE}_{2}$. This suggested that prostaglandins play a major role in the control of breathing activity and at the time of birth. Howewer, meclofenamate treated fetuses showed a similar significant decrease of breathing activity during the two days before delivery (Wallen et al. 1988). Therefore, the decrease of breathing activity before delivery is not dependent on the concurrent increase in $\mathrm{PGE}_{2}$. Peripheral and central $\mathrm{PGE} \mathrm{E}_{2}$ increased remarkably just before delivery and decreased during the first 24 hours rapidly after delivery. However, the establishment of continuous breathing at birth occurred when $\mathrm{PGE}_{2}$ levels (peripheral or ventriculocisternal) were similar or higher than fetal levels (Jones et al. 1990, Lee et al. 1989). This suggests that the fall in $\mathrm{PGE}_{2}$ level is not the primary mechanism involved in the establishment of continuous breathing at birth, but may play a role in the maintenance of continuous breathing.

In summary, a number of pharmacological agents stimulate breathing activity such as pilocarpine, opiates, doxapram, theophylline, caffeine, 5 -hydroxytryptophan, TRH. Fetal breathing activity is depressed when the fetus is exposed to pentobarbitone, diazepam, adenosine (see hypoxemia) or $\mathrm{PGE}_{2}$.

Many mechanims have been studied and proposed as responsible for the regulation of breathing activity in utero. It is difficult to draw firm conclusions since the actions and proposed mechanisms vary widely. It seems highly unlikely that so many different mechanisms are involved in the normal regulation of breathing activity in utero.

\subsection{Birth}

At birth, gas excliange must be achieved by the lungs. At birth many factors are involved in the stimulation of breathing activity such as extra afferent input, e.g. cooling, touch, pain, sound and light, which can contribute to the initiation of breathing or facilitate it by changing the sensitivity $10 \mathrm{CO}_{2}$. Furthermore, in the newborn there is an increase in systemic vascular resistance, a decrease in pulmonary vascular resistance and an increase in pulmonary blood flow (Iwamoto et al. 1987). After birth there is exclusion of the placenta from the circulation and levels of inhibitory substances produced by the placenta fall which may allow continuous breathing activity (Adamson et al. 1987, Blanco et al. 1987b, Adamson et al. 1991). Umbilical cord occlusion and increased pulmonary blood flow may result in decreased level of fetal prostagladins (Adamson et al. 1991. Ferreira \& Vane 1967). This led to the suggestion that a decrease of placental factors secreted into the plasma is involved in the initiation of continuous breathing at birth. 


\subsubsection{Extra afferent input}

In utero there are only small changes in temperature and other conditions in the environment are quite stable. The change in environment at birth exposes the fetus to extra afferent input produced by cooling, touch, pain, light and sound which may play a role in the initiation of continuous breathing activity. Peripheral nerve electrical stimulation resulted in continuous breathing activity in fetal sheep (Condorelli \& Scarpelli 1975 . Scarpelli et al. 1977) but these results could not be confirmed by others (Blanco et al. 1983b). Stimulation of the fetus by sound resulted in ti significant change in ECoG state from HV ECoG to LV ECoG associated with breathing activity, rapid eye movements and nuchal muscle activity suggesting arousal (Fletcher et al. 1989, Parkes et al 1991). Extra afferent input might change thresholds and the arousability of the newborn, resulting in continuous breathing.

Newborn lambs respond to cooling by increasing pulmonary ventilation (Andrews et al. 1991) and oxygen consumption (Alexander \& Williams 1968, Dawes et al. 1968, Sidi et al. 1983, Andrews et al. 1991). The increase in ventilation was caused by an increase in breath amplitude (young lambs) or in older lambs an increased amplitude in combination with a higher frequency (Andrews et al. 1991). It has been concluded that metabolic rate is a major stimulus in modulating breathing activity (Andrews et al, 1991). When the newborn lamb was exposed to cooling, there was also an increase in heat production, in blood pressure and heart rate (Sidi et al. 1983) and in plasma norepinephrine levels (van Bel et al. 1993). These responses are mature before birth in the ovine fetus (Gluckman et al. 1983, Gunn et al. 1985, Kuwamura et al. 1986). Although thermogenic responses are not needed for fetal survival, they are essential for neonatal adaptation since the newborn is exposed to an abrupt fall in environmental temperature at birth.

Reduction of fetal skin temperature induced regular rhythmic breathing activity in utero even during HV ECoG (Harned \& Ferreiro 1973. Gluckman et al. 1983). Breathing started in externally cooled feuses before there had been a fall of fetal central temperature or amniotic temperature. It was suggested that breathing activity had been induced ats a consequence of increased afferent input from cutaneous thermoreceptors which could have resulted in arousal Interestingly, the effects were inhibited during hypoxemia which again suggested that central control of fetal breathing activity overrides peripheral control.

Slow external cooling produced by a coil around the fetus had no effect on breathing activity. Slow internal and rapid internal cooling had no effect on the periodicity of fetal breathing movements and breathing movements remaining associated with LV ECoG (Gluckman et al 1983). The results of these experiments were limited by the fact that the internal coil was in contact with the pharynx, larynx, oesophagus and ended in a loop inside the stomach. This might have influenced breathing activity during cooling periods by direct stimulation of sensitive upper atrway areas. 
The mechanism by which cooling changes the pattern of breathing in utero is not known. It coull be via direct changes in arousability as described but also one can imagine that the sensitivity to $\mathrm{CO}_{2}$ might have changed. It was reported by Moss et al. (1983) that in acute experiments the normally high $\mathrm{CO}_{2}$ threshold of the fetus $(7.4 \pm 0.3 \mathrm{kPa})$ was lowered by peripheral cooling and a decrease in core temperature. Fetal placement in a cool bath produced immediate wakefulness and onset of breathing which was attributed to a cutaneous receptor reflex. When the newborn was exposed to peripheral and central cooling there was an increase in muscle movements, shiwering, and an increase in oxygen consumption which resulted in an increase in $\mathrm{CO}_{2}$ production. More regular breathing movements occurred in association with central core cooling and peripheral cooling (Moss et al 1983). Moreover, it is reported that at birth, at the time of initiation of breathing, cold could override the negative effects of hypocapnia or moderate hypoxia (Blanco et al. 1987b).

In summary, peripheral afferent input may change thresholds involved in the initiation and maintenance of continuous breathing at birth. Thus a decreased temperature may pllay a role in the initiation of breathing activity directly or may change the sensitivity to $\mathrm{CO}_{2}$ and override the inhibitory effects of HV ECOG.

\subsubsection{Umbilical cord occllusion}

In utero gas exchange is regulated by the placenta. The placenta is known to produce many hormones which could modulate fetal breathing activity, e.g $\mathrm{PGE}_{2}$, endorphins, progesterone, adenosine etc. After cord occlusion there is a decrease in concentrations of plasma levels which could allow the establishment of continuous breathing at birth.

The existence of a placental factor modulating breathing activity in utero was proposed by Adamson et al. (1987) and Blanco et al. (1987b). In their experiments breathing activity became continuously present after cord occlusion and breathing activity ceased on the release of the cord in mechanically ventilated fetal lambs. This suggested that modulators were restored to the fetal circulation when the cond was released and that these modulators inhibited breathing activity in utero. However, during these experiments $\mathrm{PaCO}_{2}$ increased and $\mathrm{pH}$ decreased. Therefore, the presence of these confounding changes in these experiments make them inconclusive.

However, other inwestigators reported that during cord occlusions, when fetal $\mathrm{PaO}_{2}$ remained in the normoxic range $(2-3 \mathrm{kPa})$ and $\mathrm{PaCO}_{2}$ rose to $9-12 \mathrm{kPa}$, there was no continuous breathing activity (Faber et al. 1985, Baier et al. 1990, Alvarez et al. 1992). Recently, Adamson et al. attempted (1991) to keep $\mathrm{PaCO}_{2}$ unchanged during cord occlusion by using high frequency oscillation (HFO). Under these conditions cord occlusion produced an increase in the incidence, frequency and amplitude of fetal breathing movements during a 30 min cord occlusion compared to a $20 \mathrm{~min}$ control period. The increase was larger in the last $10 \mathrm{~min}$ than 
during the first $10 \mathrm{~min}$ of the $30 \mathrm{~min}$ cord occlusion. This suggests that other factors are likely to be responsible for the intiation of breathing at birth since continuous breathing activity is normally initiated within $5 \mathrm{~min}$ of cord acclusion (Adamson 1993). Since these experiments require expansion of the lung with air new variables were added to the experiments, e.g. pulmonary circulation increased and the arways were in contact with changes in $\mathrm{CO}_{2}$ (Marsland et al. 1975, Haddad Mellins 1977. Banzett et al. 1978, Sheldon \& Green 1982).

Alvaro et all. (1993) reported that they could obtain inhibition of breathing activity by infusion of a placental extract in fatal sheep. These experiments did have a lack of specificity since they did not single out a possible substance which might have been involved. The placenta could certainly contain substances which inlubit breathing activity but Alvaro et al. did not test whether extracts of other organs had an effect on fetal breathing activity, for example an extract of brain, liver etc. Furthermore, fetal $\mathrm{PaO}_{2}$ in these experiments was in the range of $27-45 \mathrm{kPa}$ a level which would never normally be reached in prenatal or postnatal life. The physiological activity of substances in the extracts at a significantly lower $\mathrm{pH}$ and an unphysiologically high $\mathrm{PaO}_{2}$ might theretiore be different from the physiological activity of substances which were released normally by the placenta in the normoxic-normocapnic fetus.

In summary, it is clear that at the moment of birth or shortly thereafter there are changes in oxygen consumption and $\mathrm{CO}_{2}$ production. These changes could certainly have an important role in the establishment and maintenance of breathing at birth. Cord occlusion experiments may have introduced confounding variables since blood gases were not well controlled and the lungs were exposed to mechanical ventilation, CPAP and the airways were exposed to changes or increased levells of $\mathrm{CO}_{2}$. Use of techniques which control fetal blood gases without pulmonary ventilation could offer the solution to the question of whether exclusion of the umbilical circulation per se, or a rise in $\mathrm{PaCO}_{2}$ during this transition, play a role in the initiation of breathing at birth. 


\title{
Chapter 3
}

\section{Materials \& Methods}

\author{
3.1 Animals \\ 3.2 Surgical procedure \\ 3.3. Extracorporeal membrane oxygenation system \\ 3.3.1 Priming of the system \\ $3.4 \quad$ Data collection \\ 3.5 Experimental protocol and analysis of results \\ 3.6 Statistics
}




\subsection{Animals}

Experiments were performed on unanesthetized chronically instrumented fetal sheep in utero. The chronic fetal sheep preparation has been used for the past 20 years to study fetal and neonatal physiology. The reason for this is that many techniques are well established and documented: the fetus is llarge enough to be instrumented, the ewe tolerates abdominal surgery well, will stand and eat withim 30 min of recovery from anesthesia, adapts well to living in metabolic cages and is not prone to abortion following surgery.

\subsection{Surgical procedure}

Pregnant sheep were operated at $128-132$ days of gestational age after 48 hours fasting using sterile technique and under general anesthesia. The anesthesia was induced with Sodium Thiopentone (Nesdonal) $1 \mathrm{~g} / 70 \mathrm{~kg}$ i.y. The ewe was intubated and maintained during the operation with $0.8 \%$ Halothane in $\mathrm{N}_{2} \mathrm{O}$ and $\mathrm{O}_{2}(50 \% / 50 \%)$. Catheters (polyvinyl, $1 \mathrm{~mm}$ ID) were placed in the ewes carotid artery and jugular vein for blood sampling or infusing antibiotics. A midline laparotomy was performed and the uterus was partly exteriorized to allow access to the fetal head. The uterus was opened and the fetus was exteriorized down to the umbilical cord. The volume required for total occlusion of an inflatable cuff occluder (NO-4,2 cm ID, Rhodes Medical Instruments, Woodland Hills, U.S.A.) was checked by inflating the occluder with saline until the lumen was closed. The occluder was placed around the umbilical cord and anchored to the abdominal skin. A fetal thoracotomy was done at the level of the 10 th intercostal space. Four wire electrodes (AS 632 Cooner Wire, Chatsworth, U.S.A.) were sewn in the diaphragm muscle to record EMG activity and thermistor (PT 100 sensors, Murata, Nijkerk Electronics, The Netherlands) was left in the pleural cavity to measure fetal central temperature. The thorax was closed. Polyvinyl catheters $(0.8 \mathrm{~mm} \mathrm{ID})$ were placed in the right brachial artery to measure blood pressure, heart rate and for fetall blood sampling. A catheter (polyvinyl, $0.8 \mathrm{~mm}$ ID) was placed in the right brachial vein to infuse antibiotics and fluids. Two wire electrodes for recording $\mathrm{ECoG}$ activity were implanted bilaterally on the fetal parietal dura through holes ( $1 \mathrm{~mm}$ ) drilled in the skull. A common electrode was sewn in adjacent tissue. Two wire electrodes were sewn into the periorbital muscles (vertical) to record eye movements. An incision was made at the level of the nuchal extensor muscles and 2 wire electrodes were sewn into these muscles to record EMG activity. A catheter (polyvinyl, $0.8 \mathrm{~mm} \mathrm{ID)} \mathrm{was} \mathrm{placed} \mathrm{in}$ the fetall trachea to measure intratracheal pressure.

The fetal lambs were heparinized (bolus $100 \mathrm{U} / \mathrm{kg}$ for the approximated weight) after isolating the carotid artery and jugular vein just before placing catheters in these vessels. A polyvinyl catheter 
(0.8 $\mathrm{mm}$ ID) was placed in the right carotid artery (directed towards the head) to measure blood pressure, heart rate and for fetal blood sampling. For the ECMO system two catheters (Biomedicus, Eden Prairie, U.S.A.) were placed at midcerwical level; one for drainage to the ECMO system (10-14 French, multiple holes) was advanced $10 \mathrm{~cm}$ into the right external jugular vein to the right atrium level and one for return of oxygenated blood to the fetus (8-10 French, end hole) was advanced $3 \mathrm{~cm}$ into the right carotid artery towards the aortic arch. These catheters were connected to $60 \mathrm{~cm}$ silastic tubes $(0.65 \mathrm{~cm} \mathrm{D}$, Medical Grade Tubing, Dow Corning, Midland, U.S.A.) capped at the end and filled with heparinized saline $(50 \mathrm{U} / \mathrm{ml})$. These tubes permitted later connection to the ECMO circuit.

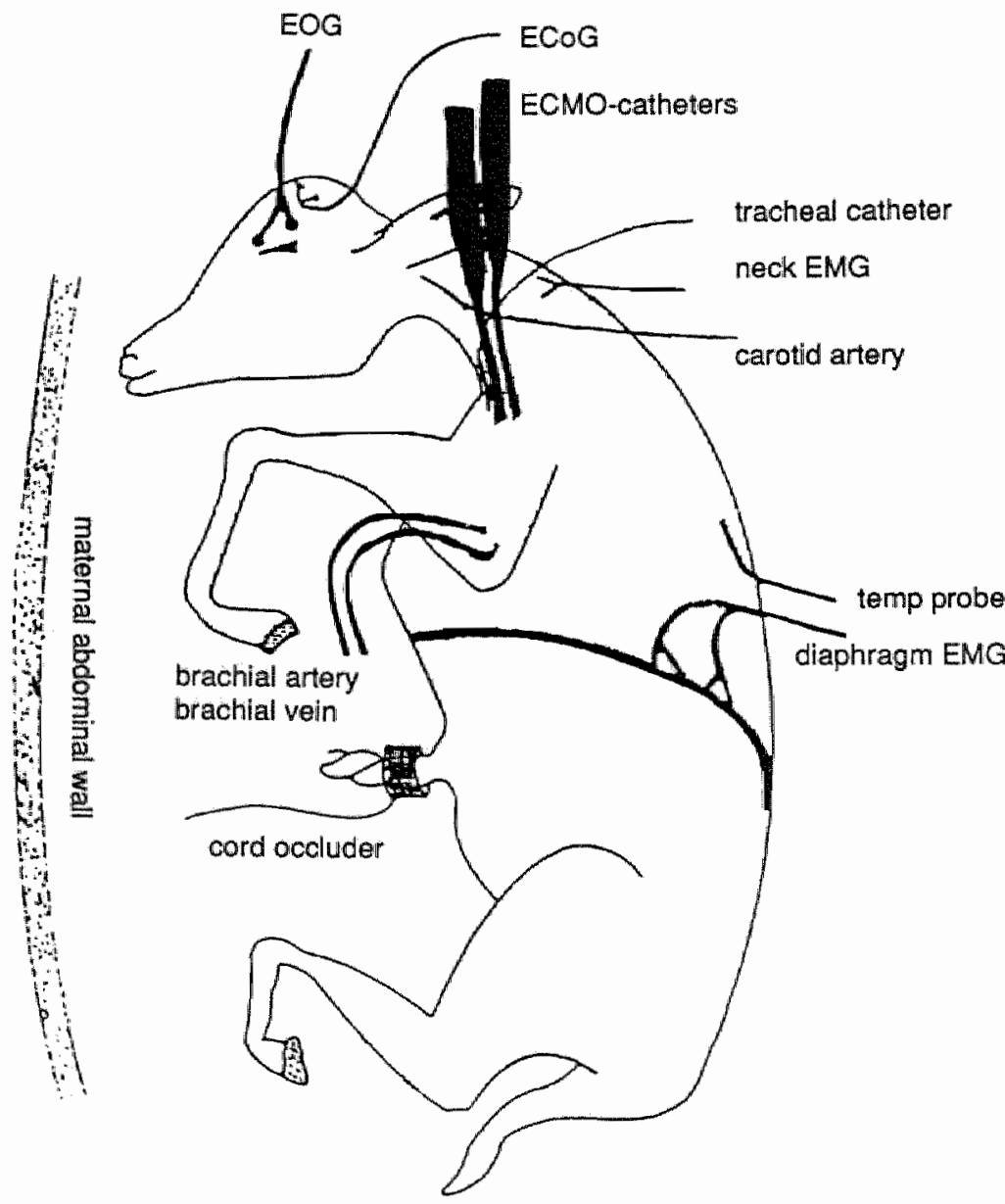


After the instrumentation was completed, the fetus was returned to the amniotic cavity. Amniotic fluid loss was replaced by warm saline. The uterus was sewn in 2 layers. All catheters, tubing and electrodes cables were exteriorized through a small incision in the flank of the ewe and stored in a plastic/cotton bag sewn adjacent to the exteriorized site. The abdomen was sewn in layers. The procedure lasted for aloout 3-3.5 hours. Figure 3.1 shows an instrumented fetus.

Postoperatively, the ewe was placed in a cage in the experimental room. The ECMO catheters were flushed twice daily with heparinized saline $(5 \mathrm{ml}, 50 \mathrm{U} / \mathrm{ml})$ and both catheters were connected to a continuous infusion of heparinized saline $(1 \mathrm{ml} / \mathrm{hr}, 50 \mathrm{U} / \mathrm{ml})$. Antibiotics were given daily to the ewe (ampicillin $1 \mathrm{~g} / \mathrm{day}$ ) and the fetus (ampicillin $150 \mathrm{mg} / \mathrm{kg} /$ day and gentamicin $5 \mathrm{mg} / \mathrm{kg} / \mathrm{day}$ ) until the end of experimentation.

The success rate of this preparation was reasonable ( $50 \%$ ). During surgery placing of the ECMO catheters was sometimes difficult due to their size and stiffness after resterilization. Another reason for failure of the preparation was hemorrhage, due to heparinization. Furthermore, despite the fact that the ewes were not prone to abortion after surgery, some ewes were in labour at the time of experimentation due to the timing of the surgery (third trimester).

\subsection{Extracorporeal membrane oxygenation system}

The ECMO system is essentially a modified heart-lung machine. The system consisted of $0.65 \mathrm{~cm}$ ID silastic tubing (Medical Grade Tubing, Dow Corning, Midland, U.S.A.), a venous reservoir (bladder, $50 \mathrm{ml}$ silastic reservoir, Scimed Life systems, Minnesota, U.S.A.), a raceway (silastic tubing exposed to the roller heads of the pump, $0.95 \mathrm{~cm} \mathrm{ID,}$ Medical Grade Tubing, Dow Corning, Midland, U.S.A.), a peristaltic pump (Travenol), a membrane lung (Scimed $0.8 \mathrm{~m}^{2}$, Scimed life systems, Minnesota, U.S.A.), connectors (Baxter, Uden, the Netherlands), stopcocks (Baxter, Uden, the Netherlands), and pigtails (stopcock with $10 \mathrm{~cm}$ extension, Vigo-Spectromed, Heisingborg, Sweden). The circuits were made according figure 3.2 and later sterilized (the membrane lung was delivered sterile). Three venous ports before the roller pump were. placed in the circuit. One port was for temperature measurement of the blood, one port was for venous sampling and one port was for continuous infusion of heparin. One venous port was placed in the circuit before the membrane for pre-membrane pressure monitoring. One arterial port was placed after the membrane for post-membrane monitoring.

\subsubsection{Priming of the Circuit}

There are 3 phases of priming procedure: gas prime, crystalloid prime and blood prime. For the gas prime, $\mathrm{CO}_{2}$ is used since $\mathrm{CO}_{2}$ replaces $\mathrm{N}_{2}$ and $\mathrm{CO}_{2}$ is more soluble in liquid. The crystalloid prime is used to fill the 


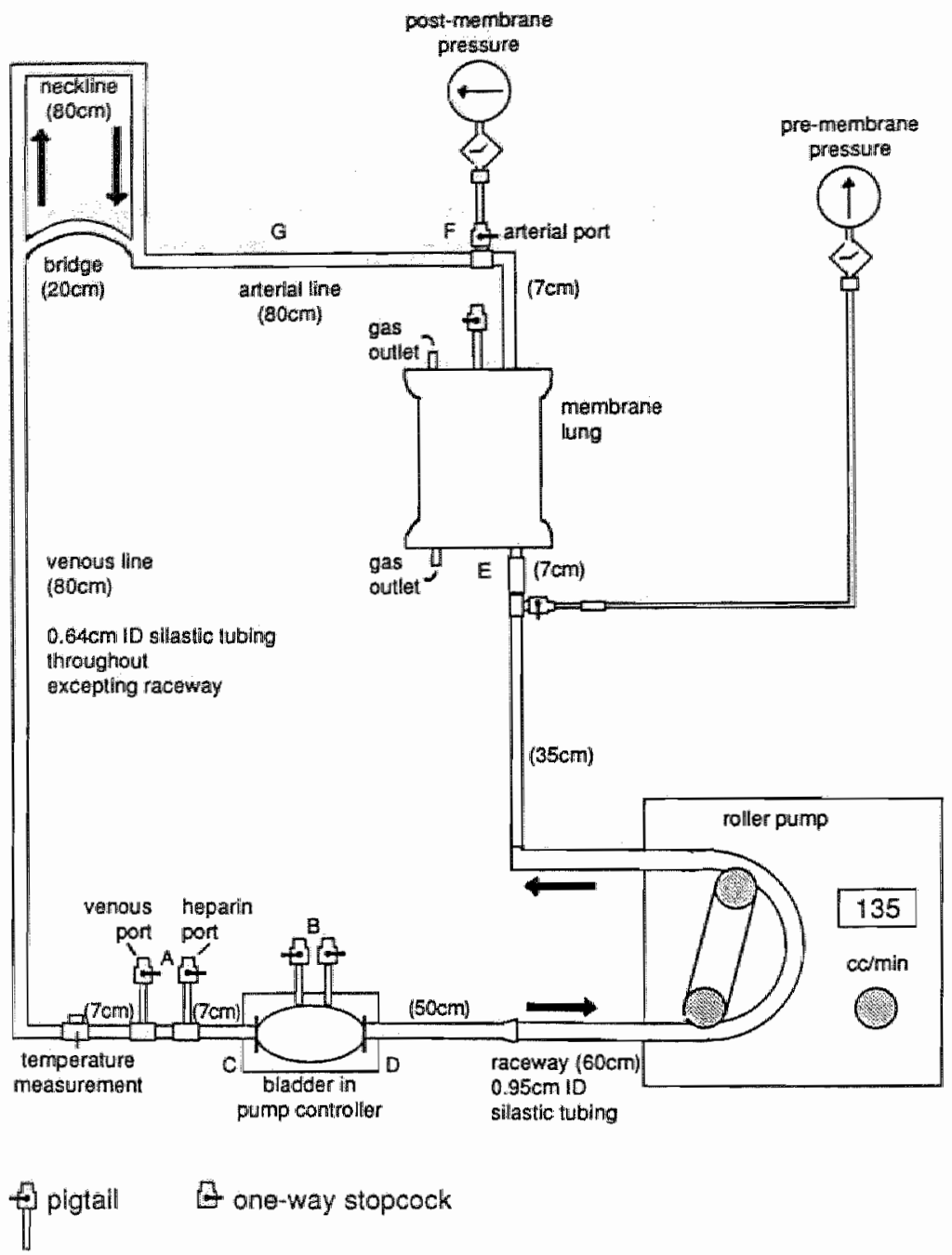

Diagram of the ECMO clrcuit made for the experiments. The capital letters correspond to specific tasks in the priming procedure.

circuit with a balanced electrolyte solution in a bubble free way and to coat the circuit.

\section{The gas prime}

$\mathrm{CO}_{2}$ was introduced in the blood path of the circuit at a flow of $2 \mathrm{l} / \mathrm{min} \mathrm{CO}_{2}$ via a filter connected to one of the stopcocks (B) of the bladder and at the same time the arterial port (F) was open. After $3 \mathrm{~min}$ a clamp was placed on the arterial line $(G)$ between the arterial port and the 
bridge. Therefore, $\mathrm{CO}_{2}$ passed through the membrane lung. Three minutes later the $\mathrm{CO}_{2}$ flow was stopped and the stopcock $(\mathrm{B})$ of the bladder and the arterial port (F) were closed simultaneously. The clamp was removed. Wall suction was connected to the gas inlet and the gas outlet of the membrane lung. Applied suction did not exceed $30 \mathrm{mmHg}$ to prevent damaging the silicone membrane. At this point the bladder collapsed.

\section{The crystalloid prime}

Haemaccel (Behring, Pharma Hoechst, the Netherlands) was used to fill the system, connected via an infusion set to a stopcock (B) of the bladder. One clamp was placed on the inlet (C) of the bladder and one clamp on the outlet (D) of the bladder. The stopeock (B) was opened and the bladder was filled with Haemaccel. The negative pressure within the system generated by wall suctioning drove this part of the priming. The circuit was filled in two parts, retrograde up to the arterial port (F) and antegrade through the membrane till the arterial port $(F)$.

Retrograde: the clamp on the inlet (C) of the bladder was opened slowly to avoid bubble formation. The venous line, neckline, bridge and arterial line were filled until the arterial port (F).

Antegrade: the outlet clamp was removed, the raceway was filled and the membrane was filled slowly. After the membrane was filled completely with Haemaccel, the line was filled till the arterial port (F). Suction was disconnected. Gentle tapping on the membrane mobilized any trapped air in the membrane. By briefly opening each stopcock of the pigtails each pigtail was filled with Haemaccel. All clamps were removed from the circuit, the raceway was placed in the pump, the pump was started, the prime was circulated.

\section{The blood prime}

One unit of fresh anticoagulant citrate dextrose (ACD) adult sheep blood (non-pregnant) was prepared. The blood was prepared by adding $25 \mathrm{ml}$ of tromethamine (THAM), $20 \mathrm{ml}$ of $\mathrm{NaHCO}_{3}(4.2 \%), 50 \mathrm{ml}$ of $20 \%$ albumin, 100 units of heparin to each unit. After rocking the bag thoroughly but gently, $300 \mathrm{mg}$ Calcium gluconate was added to each bag. Calcium gluconate was always added after heparin since it reverses anticoagulant activity in the blood.

The blood bag was connected by a new infusion set to one of the stopcocks (B) of the bladder. An empty bag was connected wia a connecting system to the heparin port (A). A clamp was placed on the inlet (C) of the bltudder, the stopcock (B) to the bloodbag was closed, and the stopcock (A) to the empty bag open. The pump was started carefully, until collapsing of the bladder, then the pump was turned off. A clamp was placed on the outlet (D) of the bladder, the stopcock (B) to the bladder was opened and the bladder was filled with blood. When the bladder was full with blood the clamp on the outlet (D) of the bladder was removed, and the pump turned on $50 \mathrm{ml} / \mathrm{min}$. This part of the priming is pump-driven. The blood was circulating slowly into the circuit, 
and a Haemaccel-blood interface was maintained. The flow of blood was traced along the arterial line $(G)$ to the bridge and the bridge was filled and clamped. The blood was traced along the necklines and back down to the heparin port (A). When the Haemaccel had been totally replaced by blood and extra blood was purged at the heparin port (A), the pump was turned off, the stopcock of the heparin port (A) and the stopcock (B) of the bladder were closed. All clamps were removed, the pump was turned to $200 \mathrm{ml} / \mathrm{min}$. The system was checked for air and all bubbles were removed.

The pre- and post-membrane pressures were connected and monitored. Monitoring was useful for the following reasons. Transmembrane pressure was usually between $50-150 \mathrm{mmHg}$. An acute rise in pre-membrane pressure, not associated with an increase in pump flow, was predictive of clots forming in the membrane. Post-membrane pressure was indicative of obstructions on the arterial side of the circuit, for example kinking of the arterial side or clots in the arterial catheter.

Additional volume was added to the circuit to bring the postmembrane pressure to $100 \mathrm{mmHg}$ at a pump flow of $200 \mathrm{ml} / \mathrm{min}$. These adjustments prevented nett loss of wolume from the fetus into the circuit at the time of initiating bypass.

Finally, the gas flow was connected to the membrane lung. In order to maintain fetal normocapnia and normoxia after connection of the fetus to the $\mathrm{ECMO}$ circuit the membrane lung was supplied with $0.4 \mathrm{~V} / \mathrm{min} \mathrm{O}_{2}$ $1.5 \mathrm{l} / \mathrm{min} \mathrm{N}_{3}$ and $0.1 \mathrm{l} / \mathrm{min} \mathrm{CO}_{2}$ at a total flow rate of $2 \mathrm{l} / \mathrm{min}$. The circuit was enclosed in a thermostatically controlled box which maintained the blood at $39.5{ }^{\circ} \mathrm{C}$ (equivalent to fetal central temperature).

The neckline was clamped with 2 clamps; one on the venous side and one on the arterial side. Both the ECMO catheters were clamped, and cleaned with $75 \%$ ethyl alcohol. The silastic tubing of the venous ECMO catheter was connected to the venous side of the neckline with a connector (Baxter, Uden, the Netherlands), the silastic tubing of the arterial ECMO catheter was connected to the arterial side of the neckline with a connector. The clamps of the ECMO catheters were removed. The pump flow was set to $50 \mathrm{mil} / \mathrm{min}$, the clamp on the arterial neckline was removed, the main bridge was clamped and the clamp on the venous line was removed. The blood was drained from the right atrium via the jugular vein down the wenous line to the bladder, passed the roller pump, the membrane lung and returned via the arterial line through the common carotid catheter to the aorta (figure 3.3). Slowly the pump flow was increased to approximately $150-200 \mathrm{ml} / \mathrm{min}$ within $15 \mathrm{~min}$. At this flow the ECMO system took over ca. $15-20 \%$ of the fetal combined ventricular butput (Cohn et al. 1974).

Heparinized $(150 \mathrm{U} / \mathrm{ml})$ saline was infused continuously via the heparin port at a flow of $2 \mathrm{ml} / \mathrm{hr}_{n}$ to maintain activated coagulation time (ACT) at 250-350 seconds. 


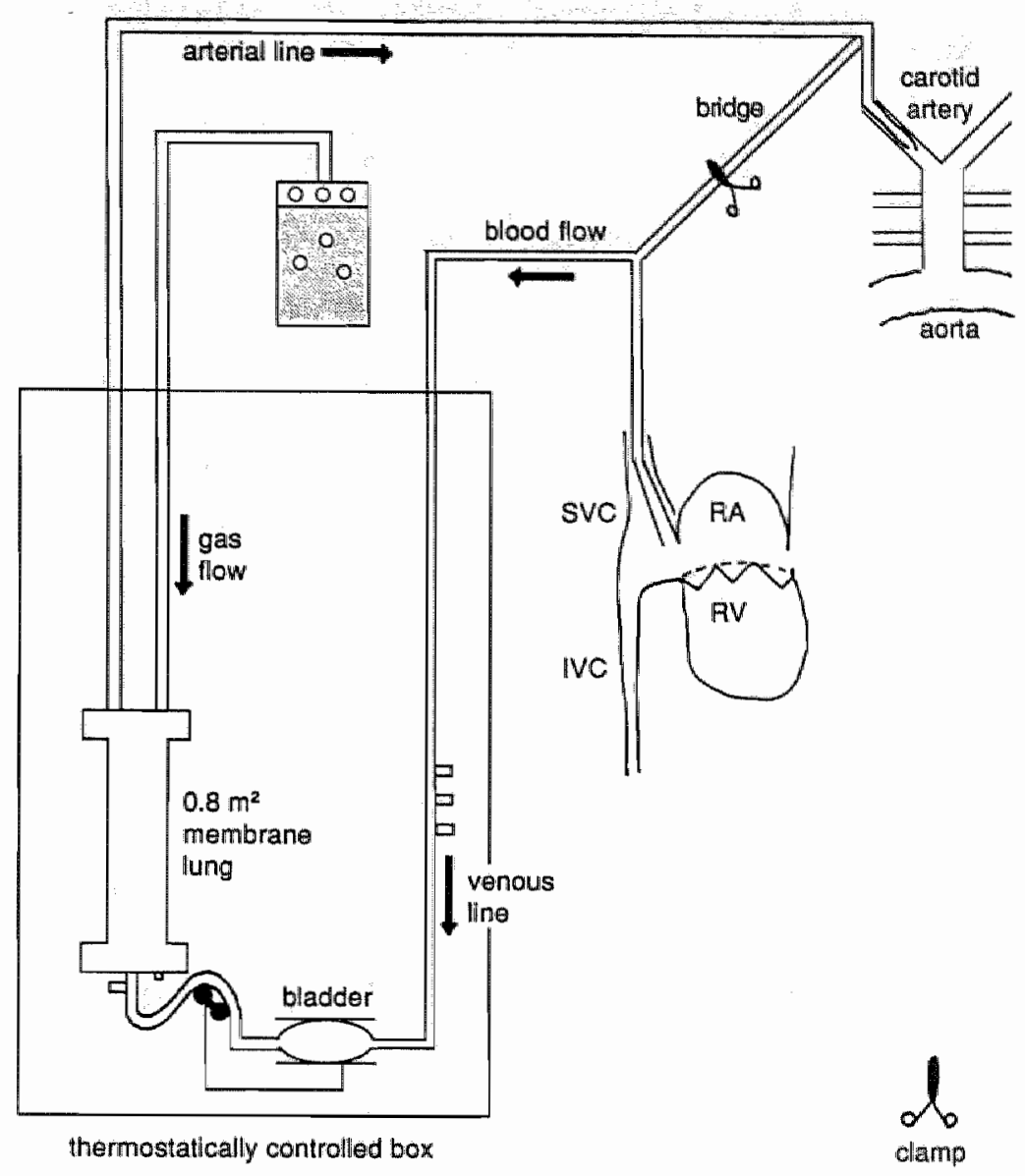

Figure 3.3

Diagram of the ECMO-system used for the experiments. Wenous blood was drained from the right atrium and followed the venous line lo the bladder, the roller pump, the membrane lung and then retumed via the arterial line into the callotid artery of the latus.

\subsection{Data collection}

Recordings were started within 48 hours after the operation. This was 24 hours before connection to the ECMO system. We used an 8 channel pen recorder (Hewlett-Packard; $7758 \mathrm{~A}$ recorder) at a paper speed of $1 \mathrm{~cm} /$ minute and the signals were also stored on tape (Hewlett-Packard 3968 A) for further analysis. 
The following parameters were recorded continuously

1 Electrocortical actiwity. obtained from bipolar electrodes amplification for scale $100 \mathrm{uV} / 2 \mathrm{~cm}$ (filters $4-40 \mathrm{Mz}$ ).

2 Integrated electromyographic activity of the posterior neck muscles (filters: 100-1000 $\mathrm{Hz}$, raw signal integrated with a leaky integrator, integrator time constant $1 \mathrm{sec}$ ).

3 Electromyographic activity of the diaphragm muscle (filters: $100-1000 \mathrm{~Hz}$, raw signal integrated with a leaky integrator, integrator time constant $0.3 \mathrm{sec}$ )

4 Electromyographic activity of the periorbital muscles (filters $0,5-40 \mathrm{~Hz})$

5 Tracheal pressure, scale $0-40 \mathrm{mmHg}$, where negative pressure deflections indicated breathing movements.

6 Blood pressure; measured by an artery catheter, scale $0-100 \mathrm{mmHg}$.

7 Heart rate; obtained from the pulse pressure signal of the blood pressure, scale 0-400 bpm.

8 Central temperature; measured from a thermistor in the pleural cavity, scale $20-50^{\circ} \mathrm{C}$, and digitalized.

Blood samples $(0.4 \mathrm{ml})$ were drawn from an arterial catheter to measure blood gas tensions and $\mathrm{pH}$ by using standard electrodes (ABL-3, Radiometer, Copenhagen) with values corrected to fetal vemperature $\left(39.5^{\circ} \mathrm{C}\right)$. Blood samples were drawn $(0.4 \mathrm{ml})$ from the venous port to measure ACT (Hemochron blood coagulation tubes P214, Hemochron $400)$.

\subsection{Experimental protocol and analysis of results}

Both integrated EMG activity of the diaphragm and the negative tracheal pressure deflections represented respiratory output. Fetal breathing was defined when activity of the diaphragm and/or repeated negative deflections of tracheal pressure were present for at least one minute (>6 breaths/min). Fetal ECoG was analyzed visually into LV ECoG and HV ECoG activity.

These recordings allowed to us obtain baseline data on incidence of fetal breathing activity, its relationship with LV ECOG activity and the distribution of fetal states before connecting the fetus to the ECMO system. A typical recording of a fetus 48 hours after surgery is shown in figure 3.4. On the third day post-surgery, when the fetal blood gases were within the normal range and when a baseline recording of $>10$ hours had shown that fetal breathing movements were present at a normal incidence, the fetuses were connected to the ECMO system, maintaining fetal temperature, blood gases and $\mathrm{pH}$. Baseline recordings on ECMO were started at least one hour after connection to the ECMO system. 


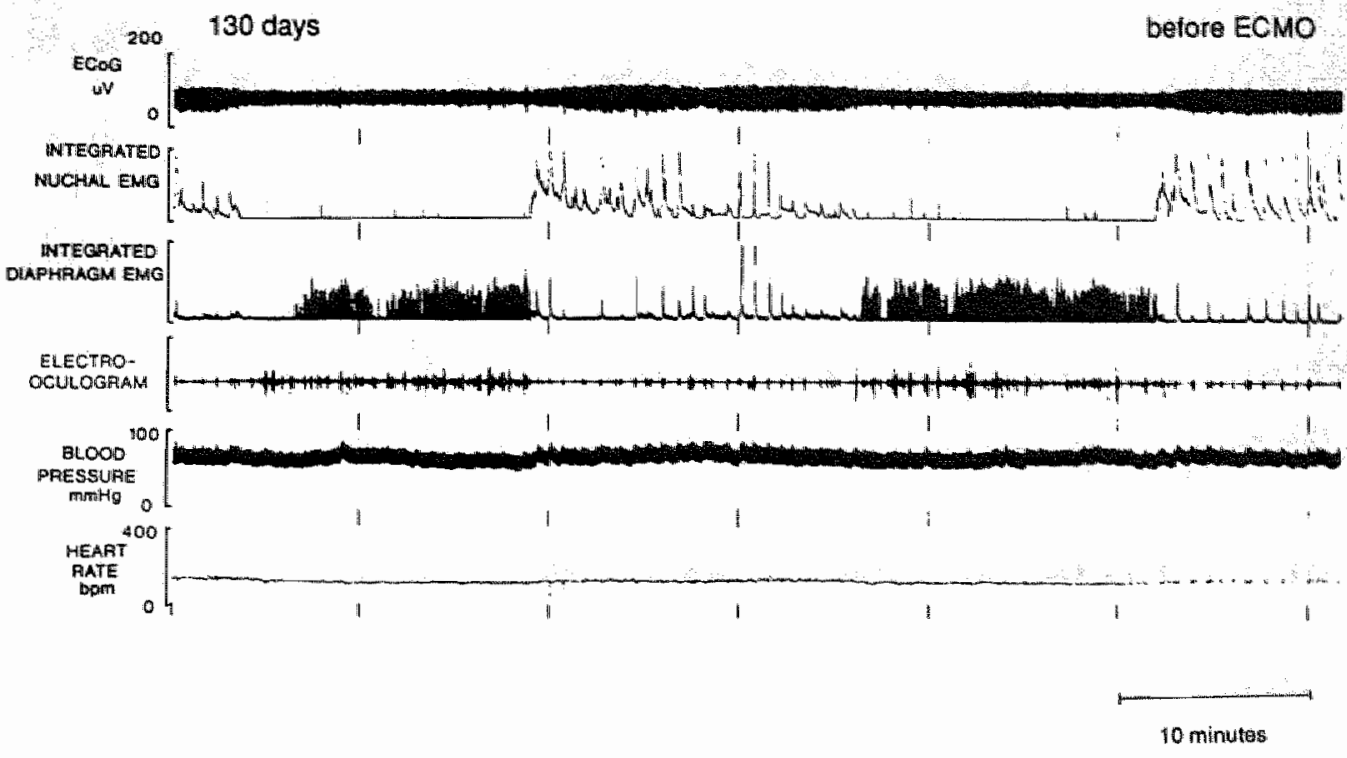

Figure 3.4

Typical recording of approximately one hour of a fetus of 130 days $\mathrm{QA}, 48$ houts after the operation. Traclings are from top to bottom: ECoG activity, integrated nuchal EMG, integrated diaphragmatic EMG, electro-aculogram, blood pressure and heant rate. $E C O G$ activity is differentiated into LV ECOG and HW ECOG activity. Fetal breathing movements and rapid eye movements are assoclated with LV ECOG activily, and nuchal EMG activity is asisociated with HV ECOG.

\subsection{Statistics}

All data are given as means \pm SEM. In statistical analysis of data $n$ was taken as the number of fetuses, not the number of experiments. Repeated trials on individual fetuses were averaged before statistical analysis.

Non-parametric tests, i.e. Friedman and Wilcoxon signed rank test, were used for statistical comparison between the periods of the protocol. 


\section{Chapter 4}

\section{The effect of mild hypocapnia on breathing and behavior in unanesthetized normoxic fetal lambs}

I.M. Kuipers, W.J. Maertzdorf, D.S. de Jong', M.A. Hanson ${ }^{2}$ and C.E. Blanco.

Dept. of Neonatology, Dept. of Cardiothoracic Surgery \& Dept. of Extra Corporeal Circulation', Academic Hospital Maastricht, University of Limburg, Maastricht, the Netherlands, Dept. of Obstetrics \& Gynaecology ${ }^{2}$, University College Londan, London, United Kingdom.

J. Appl. Physiol. 76: 1476-1480, 1994. 
Wh: $\quad$ S 


\section{Abstract}

We hypothesized that the level of $\mathrm{PaCO}_{2}$ affects the incidence of fetal breathing movements and ECoG states in chronically instiumented fetal sheep.

6 Fetuses at 128-132 days gestation were instrumented for recording fetal behavior and for later connection to an ECMO system to change fetall blood gases. Before ECMO fetal arterial $\mathrm{pH}$ and blood gases were: $\mathrm{pH} 7.40 \pm 0.01, \mathrm{PaCO}_{2} 5.7 \pm 0.20 \mathrm{kPa}$ and $\mathrm{PaO}_{2} 2.56 \pm 0.23 \mathrm{kPa}$ during ECMO in normocapnia they were: $\mathrm{pH} 7.37 \pm 0.01$;

$\mathrm{PaCO}_{2} 6.14 \pm 0.09 \mathrm{kPa}$ and $\mathrm{PaO}_{2} 3.68 \pm 0.40 \mathrm{kPa}$ during ECMO in nild hypocapnia they were: $\mathrm{pH} 7.47 \pm 0.01, \mathrm{PaCO}_{2} 4.71 \pm 0.23 \mathrm{kPa}$ and $\mathrm{PaO}_{2} 3.55 \pm 0.23 \mathrm{kPa}$.

The overall incidence of breathing movements, the incidence of breathing movements during LV ECoG and the mean duration of periods of breathing decreased significantly during hypocapnia. Fetal ECoG activity showed normal cycling during the periods of mild hypocapnia and the mean duration of LV ECoG periods did not change. During mild hypocapnia, rapid eye movements $(n=3)$ remained associated with LV ECOG and nuchal muscle activity with HV ECoO. These results suggest that the presence of breathing movements in fetal life is not only dependent on the behavioral state but also on the level of fetal $\mathrm{PaCO}_{2}$.

\section{Introduction}

Fetal breathing movements are present from early in gestation (Cooke \& Berger 1990, Dawes et al 1972, Szeto et all 1992) and are associated with phasic electro-ocular and tonic nuchal muscle activity (Clewlow et al. 1983). The function and regulation of these fetal breathing movements are unknown since gas exchange is regulated by the placenta. It hats been suggested that fetal breathing mowements are necessary for normal lung development but the mechanism is unknown (Fewell et all 1981 a Liggins et al, $198 \mathrm{lb}$ ). The first clear evidence of some regulation of breathing activity in utero occurs at the time of maturation of electrocontical activity into LV ECoG activity and high voltage electrocortical HV ECoO activity (Dawes et al. 1972, Ioffe et al. 1987). Breathing movements are present for only $30-40 \%$ of the total time and are associated with rapid eyc movements and LV ECoG (Dawes et al. 1972). During LV ECoG breathing movements are present for approximately $65 \%$ of the time (Ioffe et al. 1980) while during HV ECoG breathing movements are inhibited (Dawes et all. 1983). Thus breathing movements have been suggested to be part of the expression of fetal behavior (Dawes et atl. 1983, Jansen et al. 1981).

It is well known that the level of $\mathrm{PaCO}_{2}$ regulates breathing activity postnatally (Berger et al. 1977). It has also been demonstrated that oscillations in $\mathrm{PaCO}_{2}$ level are important in the regulation of breathing 
after birth (see Kolobow et al. 1977, Phillipson et al. 1981). However, the rolle of the $\mathrm{PaCO}_{2}$ level in determining the incidence of fetal breathing activity in late gestation, when breathing activity became episodic, is not firmly established. It is well known that hypereapnia stimulates breathing from early in gestation (Boddy et al 1974, Connors et al. 1988, loffe et al. 1987). If has been shown that matemal hypocapnia produced by hyperventilation results in a decrease of breathing activity of the human and sheep fetus during the last weeks of gestation (Boddy et al. 1974, Connors et al. 1988, Marsál et all 1979). However, hypocapnia is known to cause a decrease in uterine (Oakes et al. 1976, Walker et al. 1976) and in umbilical (Oakes et al. 1976) blood flow. Thus the fetus might have been hypoxemic (Oakes et all 1976) and this is known to inhibit fetal breathing movements (Boddy et al. 1974 , Koos et al. 1987a). In order to avoid these confounding variables we used an ECMO system in chronically instrumented fetal lambs. This allowed us to decrease fetal $\mathrm{PaCO}_{2}$ while maintaining fetal oxygenation independently of the ewe. This allowed us to address the hypothesis that the $\mathrm{PaCO}_{2}$ level in utero determines the incidence of fetal breathing movements.

\section{Materials and methods}

Experiments were performed on unanesthetized chronically instrumented fetal sheep in utero (see Chapter 3).

Recordings were obtained before ECMO, and on $\mathrm{ECMO}$ during normocapnia $\left(\mathrm{PaCO}_{2}\right.$ between $5.5-6.5 \mathrm{kPa}$ ) and mild hypocapnia periods. Mild hypocapnia was obtained by decreasing the $\mathrm{CO}_{2}$ flow to the membrane lung. Mild hypocapnia was defined as a fetal $\mathrm{PaCO}_{2}$ 1 to $2 \mathrm{kPa}$ less than baseline.

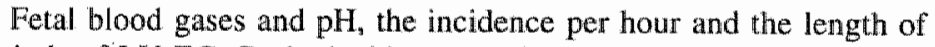
periods of $\mathbb{L V}$ ECoG, the incidence per hour and length of periods of fetal breathing movements, and the incidence of fetal breathing movements during LV ECoG were measured during a) periods before ECMO, and on ECMO b) during normocapnia periods and c) mild hypocapnia periods. Recordings also continued for a period after return to fetal normocapnia. Mean arterial blood pressure and heart rate were analyzed every $10 \mathrm{~min}$. Amniotic pressure was not subtracted from the blood pressure.

The Friedman and Wilcoxon signed rank test were used for statistical comparison of variables between the periods of the protocol. 


\section{Results}

Experiments were performed on 6 fetal lambs, at gestational age of 131-135 days.

\section{Baseline recordings before connecting the fetus to the ECMO system.}

Baseline recordings were obtained 55 to 70 hours after the operation, the duration of these recordings being 11 hrs to 21 hrs. Fetal pH and blood gases were: $\mathrm{pH} 7.40 \pm 0.01, \mathrm{PaCO}_{2} 5.70 \pm 0.20 \mathrm{kPa}$, and $\mathrm{PaO}_{2}$ $2.56 \pm 0.23 \mathrm{kPa}$. Mean arterial blood pressure was $67.2 \pm 4.3 \mathrm{mmHg}$, and heart rate was $157 \pm 9$. $1 \mathrm{bpm}$.

Nuchal EMG activity was associated with HV ECoG. Rapid eye movements ( 3 fetuses) were associated with LV ECoG activity and fetal breathing movements. LV ECoG activity occurred $49.3 \pm 2.4 \%$ of the time and the duration of the $\mathrm{LV}$ ECoG periods was $14.6 \pm 0.6 \mathrm{~min}$. Fetal breathing activity was present for $36.0 \pm 2.8 \%$ of the time, the length of the fetal breathing periods being $10.2 \pm 1.1 \mathrm{~min}$. The incidence of breathing activity during LV ECoG was $72.8 \pm 3.6 \%$.

\section{Normocapnia periods on ECMO}

On the third day post-surgery the fetuses were connected to the ECMO system. These periods of normocapnia were studied to control for possible effects of ECMO on normal fetal behaviour. Fetal $\mathrm{pH}$ and blood gases during these baseline recordings on the ECMO system were: $\mathrm{pH} 7.37 \pm 0.01, \mathrm{PaCO}_{2} 6.14 \pm 0.09 \mathrm{kPa}$, and $\mathrm{PaO}_{2} 3.68 \pm 0.40 \mathrm{kPa}$. Mean arterial blood pressure was $72.8 \pm 5.9 \mathrm{mmHg}$ and heart rate was $168.4 \pm 10.7 \mathrm{bpm}$.

Nuchal EMG activity was associated with HV ECoG activity. Rapid eye movements $(n=3)$ were associated with $L V$ ECoG activity and fetal breathing movements. "The incidence of $\mathrm{LV}$ ECoG was $46.6 \pm 2.5 \%$ of the time and the length of the LV ECoG periods was $15.5 \pm 1.1$ min (see table 4.1). Breathing activity was present for $34.6 \pm 3.0 \%$ of the time, the length of fetal breathing periods being $9.3 \pm 1.2 \mathrm{~min}$. The incidence of breathing activity during $\mathrm{LV}$ ECoG was $75.5 \pm 2.6 \%$.

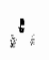


The effect of mild hypocapnia on fetal blood gases and $\mathrm{pH}_{3}$ fetal behavior and breathing activity in unanesthetized fetal lambs.

Physiologic variables Normacapnia on ECMO Mild hypocapnia on ECMO

\begin{tabular}{|c|c|c|}
\hline $\mathrm{pH}$ & $7.37 \pm 0.01$ & $7.47 \pm 0.01^{1}$ \\
\hline $\mathrm{PaCO}_{2}$ (kPa) & $6.14 \pm 0.09$ & $4.71 \pm 0.23^{\prime \prime}$ \\
\hline $\mathrm{PaO}_{2}(\mathrm{kPa})$ & $3.68 \pm 0.40$ & $3.55 \pm 0.23$ \\
\hline LV ECOG $(\%)$ & $46.6 \pm 2.5$ & $45.5 \pm 1.4$ \\
\hline LV ECOG, LP (min) & $15.5 \pm 1.1$ & $13.6 \pm 1.0$ \\
\hline FBM $(\%)$ & $34.6 \pm 3.0$ & $14.3 \pm 3.9^{3}$ \\
\hline FBM periods, LP (min) & $9.3 \pm 1.2$ & $4.9 \pm 1.2^{\prime}$ \\
\hline FBMLV ECOG $(\%)$ & $75.5 \pm 2.6$ & $32.2 \pm 9.5$ \\
\hline
\end{tabular}

Mears \pm SEM of lelal blood gases and $\mathrm{pH}_{3}$ incidence and length of periods of $L V$ ECOG and fetal breathing movements, the incidence of fetal breathing mowements during LV ECoG (FBM/LV ECoG). (LP; length of periods, FBM; fetal breathing mowements, \%: percentage of the time present, $p<0.05$, mill hypocapnia pertods on ECMO compared to nomocapnia on ECMOl.

\section{Mild hypocapnia periods on ECMO}

A total of 9 experiments were performed in 6 fetuses. Figure 4.1 shows 60 min from one experiment. There was more than one experiment performed on 3 animals: the time between experiments was 7 hours to 29 hours. The duration of mild hypocapnia periods ranged from 2 hours to 10 hours. Fetal $\mathrm{pH}$ and blood gases were: $\mathrm{pH} 7.47 \pm 0.01(\mathrm{p}<0.05$ compared to normocapnia period on $\mathrm{ECMO}$ ), $\mathrm{PaCO}_{2} 4.71 \pm 0.23 \mathrm{kPa}$ $(\mathrm{p}<0.05)$ and $\mathrm{PaO}_{2} 3.55 \pm 0.23 \mathrm{kPa}$. Mean arterial blood pressure was $69.8 \pm 3.9 \mathrm{mmHg}$, and heart rate was $164.8 \pm 14.7 \mathrm{bpm}$.

Nuchal EMG activity was present and associated with HV ECoG. Rapid eye movements $(n=3)$ were present during LV ECoG activity. The incidence of $\mathrm{LV} \mathrm{ECOG}$ was $45.5 \pm 1.4 \%$ of the time, the duration of the LV ECoG periods was $13.6 \pm 1.0 \mathrm{~min}$ (see table 4.1.). The incidence of breathing activity was $14.3 \pm 3.9 \%(p<0.05)$ and the length of the fetal breathing periods was $4.9 \pm 1.2 \mathrm{~min}(\mathrm{p}<0.05$ ). The incidence of breathing activity during LV ECoG was $32.2 \pm 9.5 \%(\mathrm{p}<0.05)$.

When fetal $\mathrm{PaCO}_{2}$ was returned to control breathing activity was present ( 2 experiments) or reappeared within 4 min ( 7 experiments). These breathing movements were only present during LV ECoG, and were stopped with the occurrence of HV ECOG activity. Figure 4.2 shows 
an example of the reappearance of fetal breathing activity after increasing fetal $\mathrm{PaCO}_{2}$. During the first hour immediately after mild hypocapnia, the incidence of breathing activity was $54.6 \pm 12 \%$ (p<0.05, compared 10 normocapnia on ECMO) and the incidence of LW ECoG was $62.4 \pm 19 \%$ $(\mathrm{p}<0.05)$. During the first hour after mild hypocapnia fetal $\mathrm{pH}$ and blood gases were: $\mathrm{pH} 7.37 \pm 0.01, \mathrm{PaCO}_{2} 6.00 \pm 0.15 \mathrm{kPa}$, and $\mathrm{PaO}_{2} 4.08 \pm 0.37 \mathrm{kPa}$.

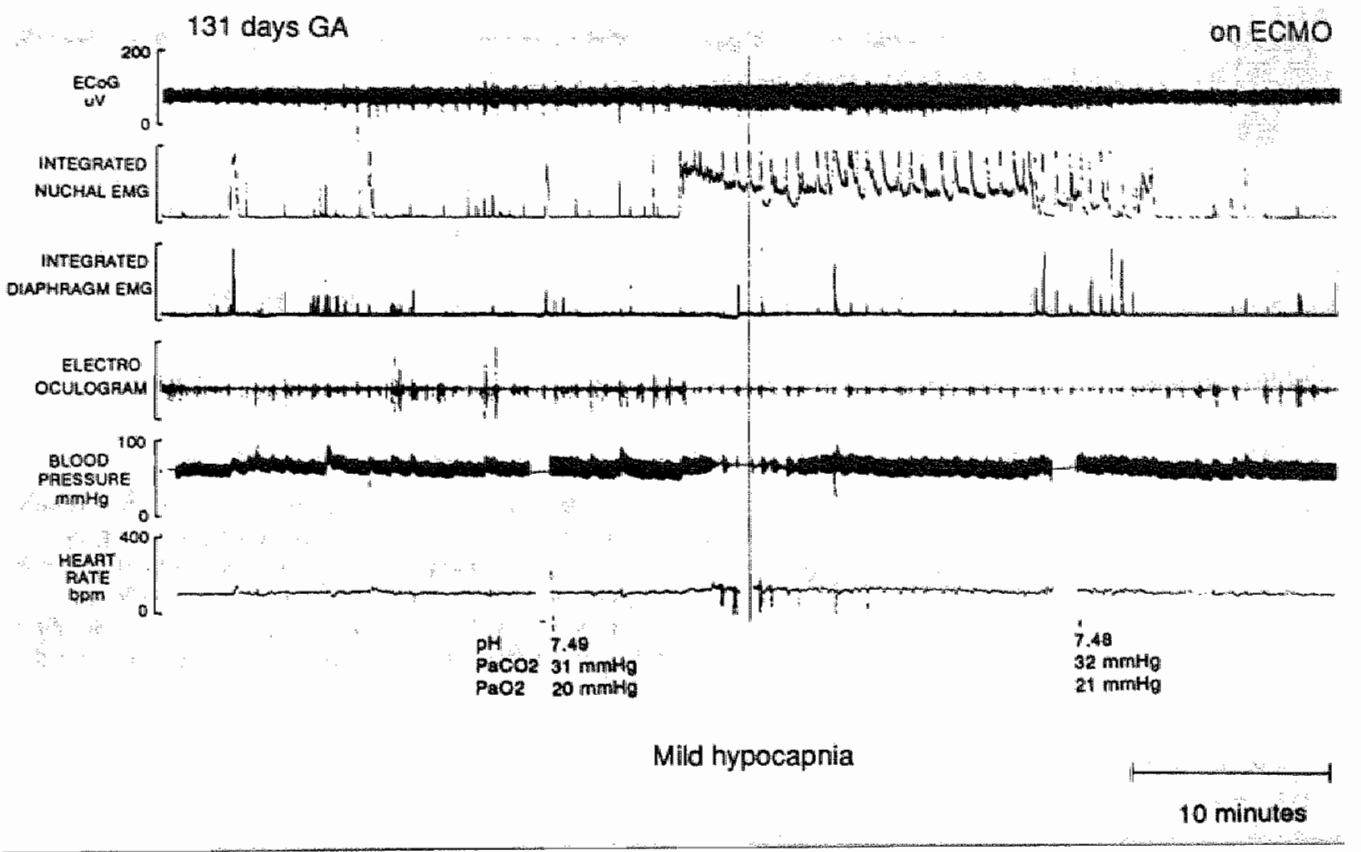

Figure 4.1

Intrauterine recording of a fetus of 131 days of gestation connected to the ECMO syman 3 days after surgery. Tracings are from the top: ECoG activity, integrated nudhal EMho integrated diaphragm EMG, electro-oculogram, blood pressure and heart rate. Blood gas and $\mathrm{pH}$ samples were taken at the indicated time. During mild hypocapmia the rewere almost no breathing movements present during LV ECoG. 


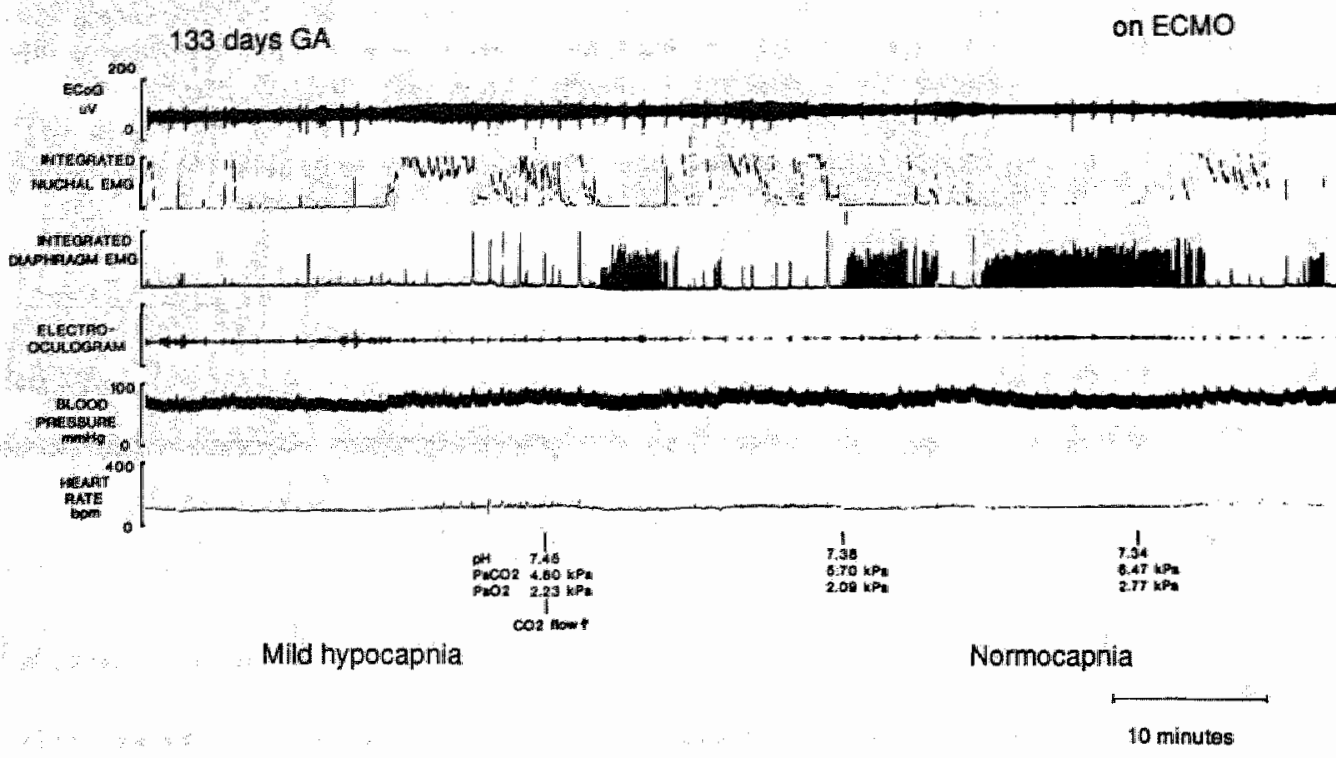

Figure 4.2

Intraterine recording of a fetus of 133 days of gestation connected to the ECMO system 4 days after surgeny. Tracings are from the top: $\mathrm{ECOG}$ activity, integrated nuchal $\mathrm{EMG}_{\text {n }}$ integrated diaphragm EMG, electro-oculogram, blood pressure, heart rate: Blood gas and $\mathrm{pH}$ samples were laken at the indicated time. During mild hypocapnia there were no breathing movements present during $L V$ ECOG. After increasing $\mathrm{CO}_{2}$ flow to the membrane to abtain fetal normocapnia, breathing movements reappeared and were associated with LV ECOG.

\section{Discussion}

The use of ECMO in utero enabled us to study the effect of mild hypocapnia during normoxia on fetal behavioral activity in fetuses of 130-134 days gestation, an age at which ECoG was differentiated. The incidence and the length of periods of fetal breathing movements decreased significantly during periods of mild hypocapnia. However, there was no change in the incidence or duration of periods of LV ECoG. Nuchall EMG and rapid eye movements were normal during mild hypocapnia periods. This suggests that lowering $\mathrm{PaCO}_{2}$ reduces the drive for fetal breathing activity.

The use of an ECMO system could by itself introduce new variables and influence fetal behavioral activity. However, we did not observe statistical differences in the incidence or duration of periods of fetal breathing movements or LV ECoG activity before or during ECMO. The fetuses showed no abnormal behavioral activity during the baseline periods on $\mathrm{ECMO}$, as LV ECoG activity remained associated with rapid eye movements and fetal breathing movements, and HV ECoG remained associated with nuchal muscle activity. Since these parameters (fetal 
breathing movements and fetal behavior) are generally used to define the "normality' of the preparation we do not consider that the use of extracorporeal eirculation by itself influenced the results.

In our experiments there was no change in the incidence or duration of periods of LV ECoG activity during hypocapnia Rapid eye movements remained associated with $\mathrm{EV} \mathrm{ECOG}$ activity and nuchal EMG activity remained associated with HV ECoG activity. Despite the fact that LV ECoG did not change, the incidence and the length of periods of breathing movements and the incidence of fetal breathing movements during LV ECOG decreased significantly during hypocapnia. This suggests that fetal breathing activity can be altered independently of other aspects of behavioral states. Although we found a significant reduction in breathing activity it was not totally abolished. Presumably this was because we used only mild hypocapna. In adult cats it has been reported that graded reduction in $\mathrm{PaCO}_{2}$ to as low as $0.5 \mathrm{kPa}$ caused a graded reduction of breathing activity to the point where breathing became irregular (Berkenbosch et al. 1984). In the fetus, we do not know whether there is a threshold below $4 \mathrm{kPa}$ at which breathing activity becomes no longer present.

Previous studies in animals and man (Boddy et al. 1974, Connors at al. 1988, Marsál et al. 1979) reported a decrease of fetal breathing activity during hypocapnia: In human studies (Connors et al. 1988, Marsál et al. 1979) fetal hypocapnia was obtained by maternal hyperventilation which limited the duration of the experiments to $15 \mathrm{~min}$ (Connors et al. 1988) or less (Marsál et al. 1979). Maternal hyperventilation and hypocapnia present several problems, in particular the decrease in uterine and in umbilical blood 1low (Oakes et al. 1976). This can result in fetal hypoxemia and acidosis (Oakes et al. 1976) although no fetal blood samples were taken in human studies. In the sheep fetus Boddy et al. (1974) reported a decrease in incidence of breathing movements during hypocapnia obtained by maternal hyperventilation for an unspecified period in 2 normoxic fetal lambs. We have now extended these observations by maintaining fetal hypoeapnia for several hours under conditions where the fetus remained normoxemic.

Whilst we maintained fetal $\mathrm{PaO}_{2}$ constant during hypocapnia, it is possible that, under these hypocapnic conditions cerebral blood flow will decrease ca. $25 \%$ (Rosenberg et al. 1982). The $\mathrm{O}_{2}$ dissociation curve will shift to the left (Bohr-effect) and pyruwate and lactate concentrations may increase in brain tissue and CSF (Rosenberg 1988. Siesjo \& Ingvar 1986). Therefore, hypocapnia may be associated with central hypoxemia. There are seweral reasons why we do not think that this was likely to have been the cause of the reduction in breathing which we observed. First, in our experiments arterial hypocapnia was only mild (mean delta $\mathrm{PaCO}_{2}$ of $1.44 \mathrm{kPa}$ ). In piglets a decrease in cerebral blood flow only occurred during severe hypocapnia $\left(\mathrm{PaCO}_{2}<2 \mathrm{kPa}\right)$ although even then there was maintenance of the flow to the brain stem (Hansen et al. 1984).

Furthermore, maintenance of oxygen consumption occurs during hypocapnia due to an increase in cerebral oxygen extraction (Rosenberg 1988). Lastly, during hypoxemia, breathing activity, nuchal EMG activity 
and rapid eye movements are inhibited (Boddy et al. 1974, Koos et al. 1987a, Woudstra et all. 1990): but in our experiments; beside the decrease in the incidence of breathing movements, we did not see such an inhibition of rapid eye movements or nuchal EMG actiwity. Therefore, we conclude that the effects on breathing were not due to central hypoxemia but to mild hypocapnia and alkalosis.

After mild hypocapnia periods, when fetal $\mathrm{PaCO}_{2}$ returned to baseline values, breathing activity reappeared within $4 \mathrm{~min}$. It remained periodic but its incidence was increased compared to the normocapnia periods. Walker et al. (1986) observed a significant increase in breathing actiwity and even breathing activity during $\mathrm{HV} \mathrm{ECoG}$ (Walker et al. 1986) after a period of 8 hours of maternal hyperthermia which produced fetal Hypocapnia. Such prolonged hypocapnia will produce a reduction in bram bicarbonate concentration, and therefore when $\mathrm{PaCO}_{2}$ is returned to normal a rellative central acidosis will result (Fencle 1986). It is known that lowering CSF $\mathrm{pH}$ or producing central acidosis using $\mathrm{NH}_{4} \mathrm{Cl}$ stimulates fetal breathing activity (Hohimer et al. 1983, Molteni et al. 1980):

In conclusion, the level of $\mathrm{PaCO}_{2}$ is an important determinant of the incidence of breathing activity in utero. This incidence can be changed independertly of that of behavioral states. The mechanism by which $\mathrm{CO}_{2}$ determines the activity of the respiratory network in the fetal brainstem has to be determined, but clearly it is a fundamental determinant of the presence of fetal breathing activity. 


\section{Chapter 5}

\section{The effect of hypercapnia and hypercapnia associated with central cooling on behavior in unanesthetized fetal lambs}

I.M. Kuipers, W.J. Maertzdorf, D.S. de Jong', M.A. Hanson ${ }^{2}$ and C.E. Blanco.

Dept. of Neonatology and Dept. of Cardiothoracic Surgery \& Dept. of Extra Corporeal Circulation', Academic Hospital Maastricht, University of Limburg, the Netherlands, Dept of Obstetrics \& Gynaecology", University College London, United Kingdom.

Submitted 


\section{Abstract}

In utero breathing activity is periodically present and it must become continuous at birth. We investigated the effect of hypercapnia and of hypercapnia combined with central cooling on fetal breathing periodicity in lambs, using an ECMO system to control fetal blood gases and fetal temperature in 7 chronically instrumented fetal lambs of 131-134 days gestation. During fetal hypercapnia $\left(\mathrm{PaCO}_{2} 7.39 \pm 0.15 \mathrm{kPa}\right)$ frequency. amplitude and incidence of fetal breathing movements during LV ECoG increased significantly compared to isocapnic control on ECMO but it remained absent during $\mathrm{HV}$ ECoG. During hypercapnia associated with central cooling $\left(\mathrm{PaCO}_{2} 7.90 \pm 0.13 \mathrm{kPa}\right.$, temperature decrease $\left.2.1^{\circ} \mathrm{C}\right)$ there were similar changes in fietal breathing movements during LV ECoG. However, in 4 out of 7 fetuses fetal breathing movements continued throughout HV ECoG. Hypercapnia associated with central cooling can thus override the inhibitory effects of HV ECOG on fetal breathing movements. This may be due to changes in $\mathrm{CO}_{2}$ sensitivity produced by an increase in afferent input to the central nervous system.

\section{Introduction}

In utero, after maturation of electrocortical activity, breathing activity is inhibited during HV ECoG (Dawes et al. 1972, Dawes et al. 1983). This inhibition must be overridden at birth to allow the establishment of contimuous breathing. The mechanisms involved in the initiation of continuous breathing at birth are not completely understood. Fetal hypercapnia, obtained by increasing maternal $\mathrm{PaCO}_{2}$ produces an increase in incidence, amplitude and frequency of fetal breathing movements only during LV ECoG (Boddy et al. 1974, Chapman et al. 1980, Ritchie \& Lakhani 1980, Bowes et al. 1981b, Dawes et al. 1982, Connors et al. 1988, Connors et al. 1989). It is known that the level of $\mathrm{PaCO}_{2}$ increases after cord occlusion at birth and an increase in $\mathrm{PaCO}_{2}$ it is important for the initiation of breathing (Blanco et al. 1987b, Berger et al. 1990).

Mechanisms involved in the inhibition of breathing during HV ECoG are not known. It is possible that changes in $\mathrm{CO}_{2}$ sensitivity could play a role. This idea could be supported by the observed continuous breathing activity obtained by a decrease in cutaneous fetal temperature (Gluckman et al. 1983). Johnston and Gluckman (1989) described continuous breathing activity ; during hypercapnia but not during normocapnia in fetal lambs with lesions in the rostral lateral pons. It could be speculated that these lesions liberated the respiratory centres from inhibitory influences present during HV ECOG. These observations support the idea that a decrease in sensitivity to $\mathrm{CO}_{2}$ could occur during $\mathrm{HV}$ ECoG in intact animals resulting in the loss of respiratory drive and apnea (Johnston \& Gluckman 1989). If this mechanism exists, it could play a role at birth 
when there is a combination of an increased afferent input, a decrease in temperature and hypercapnia.

A previous study showed that a decrease in core temperature produced by an internal coil failed to produce continuous breathing (Gluckman et al. 1983). This would not support our proposal but there are a number of other factors which could have been responsible for these results. In that study there was no change in fetal $\mathrm{PaCO}_{2}$ and the cold internal coil passing the pharynx and larynx could have stimulated the upper airway senisitive receptors inhibiting breathing (Gluckman et al. 1983). We decided to use a different approach by using an extra corporeal membrane oxygenation system with which blood gases and blood temperature can be changed. We tested the hypothesis that the association of hypercapnia with central cooling, obtained by cooling the blood of the circuit, could override the central inhibition during HV ECOG resulting in continuous breathing activity. This might be an important mechanism involved in the initiation of continuous breathing at birth.

\section{Materials and methods}

Experiments were performed on unanesthetized chronically instrumented fetal sheep in utero (Chapter 3).

Recordings were obtained before connecting the animal to ECMO, during control periods on ECMO, during hypercapnia periods and during periods of hypercapnia associated with central cooling. Recordings on ECMO were taken at least $\mathbb{L}$ hour after starting ECMO perfusion. Fetal hypercapnia was obtained by increasing the $\mathrm{CO}_{2}$ concentration of the gasflow to the membrane lung. Hypercapnia was defined as a fetal $\mathrm{PaCO}_{2}$ at least $1 \mathrm{kPa}$ higher than baseline. The core temperature of the fetus was decreased by $1-3^{\circ} \mathrm{C}$ by decreasing circuit blood temperature. This was obtained by turning off the heating element of the box containing the circuit and placing ice around the membrane lung and in contact with the circuit. It took $20-30 \mathrm{~min}$ to decrease fetal central temperature by $1{ }^{\circ} \mathrm{C}$ at a pump fow of $200 \mathrm{~m} / / \mathrm{min}$. Whenever fetal $\mathrm{PaCO}_{2}$ did not increase significantly from control $\mathrm{CO}_{2}$-flow to the membrane lung was increased to obtain the desired $\mathrm{PaCO}_{2}$ level. The moment when the experimental conditions periods of hypercapnia associated with central cooling were reached $\left(\mathrm{PaCO}_{2}\right.$ increased $1 \mathrm{kPa}$, central temperature decreased $\left.1{ }^{\circ} \mathrm{C}\right)$ was considered the starting point of the experiments.

The protocol was divided into 3 periods during ECMO a) control periods, b) hypercapnia periods, then returning to normocapnia and c) hypercapnia associated with central cooling periods. There was a minimum control period on ECMO of at least 2 hours. Hypercapnia and hypercapnia \& central cooling experimental periods were at least $\downarrow$ hour (except in one fetus in which the experiment was $30 \mathrm{~min}$ ). Fetal blood gases were taken approximately every 15 min during a control or experimental period and were averaged. The incidence per hour and length of periods of LV ECoG activity were analyzed. Furthermore, the mcidence of fetal breathing movements per hour, the incidence of fetal 
breathing movements during $\mathrm{LV} \mathrm{ECOG}$, frequency and amplitude of fetal breathing movements were analyzed: Frequency of fetal breathing movements per minute was analyzed by counting all breaths after replaying the tape at a higher paper speed. The amplitude was analyzed by measuring the area under the curve of the integrated EMG activity of the diaphragm (Quantimed 570, Leica). The area under the integrated nuchal EMG activity was determined in the same way as the area of the fetall breathing movements which resulted in percentage. The amplitude of breathing movements and the amount of nuchal muscle activity were expressed as a percentage of the mean breath amplitude and the mean nuchal muscle activity during control periods on ECMO (100\%) for each fetus. Mean arterial blood pressure and heart rate were analyzed every $10 \mathrm{~min}$ and averaged over the experimental periods. Fetal blood gases and pH were analyzed from fetal arterial blood samples with a Radioneter ABL3 at $39.5^{\circ} \mathrm{C}$. During cooling periods fotal blood gases were corrected for fetal central temperature.

All data are reported as means \pm SEM. Wilcoxon signed rank test was used for statistical comparison of variables between hypercapnia and control periods and between hypercapnia associated with cooling and hypercapnia periods.

\section{Results}

Experiments were performed on 7 fetal lambs, at gestational age 131-134 days.

Baseline recordings prior to connection to the $\mathrm{ECMO}$ system were obtained at least 48 hours (55-70 hours) after surgery for a duration of at least 11 hours. Fetal blood gases and $\mathrm{pH}$ were prior to connection to the ECMO system: $\mathrm{pH} 7.36 \pm 0.01, \mathrm{PaCO}_{2} 5.84 \pm 0.14 \mathrm{kPa}, \mathrm{PaO}_{2}$ $3.15 \pm 0.23 \mathrm{kPa}$. Mean arterial blood pressure was $67.7 \pm 3 \mathrm{mmHg}$, and heart rate was $163.8 \pm 8$ bm. Nuchal EMG was always associated with HV ECoG. Rapid eye movements $(\mathrm{n}=5)$ were always associated with LV ECoG and fetal breathing mowements. During the baseline LV ECOG occurred $52.5 \pm 2.0 \%$ of the time. Fetal breathing movements were present $36.3 \pm 3.0 \%$ of the total time and $69.4 \pm 5.4 \%$ during LV ECoG.

\section{Control periods on ECMO} On the third day, post-surgery the fetuses were connected to the ECMO
system.

In table 5.1 physiological variables are reported. Nuchal EMG activity was associated with HV ECOG; rapid eye movements were associated with LV ECoG and fetal breathing movements. 
Table 5.1

The effect of hypercapnia and hypercapnia with central cooling on behavior, blood pressure, theart rate, $\mathrm{pH}$ and blood gases of the fetus.

\begin{tabular}{|c|c|c|c|}
\hline Physiologic wariables & Control on ECMO & Hypercapnia on ECMO & $\begin{array}{l}\text { Hypercapnia \& central coaling } \\
\text { on ECMO }\end{array}$ \\
\hline $\mathrm{pH}$ & $7.36 \pm 0.01$ & $7.31 \pm 0.01^{11}$ & $7.26 \pm 0.01^{2}$ \\
\hline $\mathrm{PaCO}_{2}(\mathrm{kPa})$ & $6.18 \pm 0.11$ & $7.39 \pm 0.15^{4}$ & $7.90 \pm 0.13$ \\
\hline $\mathrm{PaO}_{2}(\mathrm{kPa})$ & $4.64 \pm 0.38$ & $5.17 \pm 0.7$ & $4.81 \pm 0.84$ \\
\hline LV ECOG $(\%)$ & $51.0 \pm 2.6$ & $49.8 \pm 3.4$ & $4.8 .4 \pm 9.7$ \\
\hline LV ECoG, LP (min) & $18.6 \pm 1.8$ & $17.2 \pm 2.3$ & $16.9 \pm 3.4$ \\
\hline FBMAHV ECOG & $0 \exp$ & $0 \exp$ & $4 / 7 \exp$ \\
\hline FBMLLV ECOG $(\%)$ & $B 3.5 \pm 2.5$ & $89.8 \pm 2.4^{\pi}$ & $92.1 \pm 4.5$ \\
\hline $\begin{array}{l}\text { Frequency FBM } \\
\text { (breaths/min) }\end{array}$ & $40.6 \pm 8.1$ & $47.5 \pm 7.6^{1}$ & $25.3 \pm 6.8^{2}$ \\
\hline Amplitude FBM $(\%)$ & 100 & $135.6 \pm 11.8^{1}$ & $132.8 \pm 13.9$ \\
\hline Area nuchal EMG $(\%)$ & 100 & $95.0 \pm 16.5$ & $191.0 \pm 30.6^{2}$ \\
\hline $\begin{array}{l}\text { Blood pressure } \\
\text { (mmHg) }\end{array}$ & $69 \pm 3.9$ & $68 \pm 2.6$ & $75 \pm 2.9^{2}$ \\
\hline Heart rate (bpm) & $172 \pm 10.9$ & $168 \pm 8.9$ & $210 \pm 9.5^{a}$ \\
\hline
\end{tabular}

Means $1 \mathrm{SEM}$ of hetal blood gases and $\mathrm{pH}$, Incidence and length of periods of LV ECoG and the lncidence of fetal treathing movements during LV ECOG (FBM/LV ECOG). frequency and amplitude of fetal breathing mowements, area of nuchall EMG, blood prassiutie and heart rate. Furthermore the presence of fetal breathing movements during HW ECoG. (LP" length of period, FBM; fetil breathing movements "\%; percentage of the time present or percentage increase of amplitude of fetal breathing movements or area of nuchal muscle activity, exp; experinents "p<0.05, compared to control on ECMO.

2p $<0.05$, compared to hyporcapnia on EOMO).

\section{Hypercapnia periods on ECMO}

Nuchal EMG activity was present and associated with $\mathrm{HV}$ ECoG, the area of nuchal muscle activity was not different from control on ECMO.

Compared to control periods on ECMO there was a significant increase in amplitude, frequency and incidence of fetal breathing movements during LV ECoG. However, breathing movements remained periodically present associated with rapid eye movements and LV ECoG (see figure 5.1). 

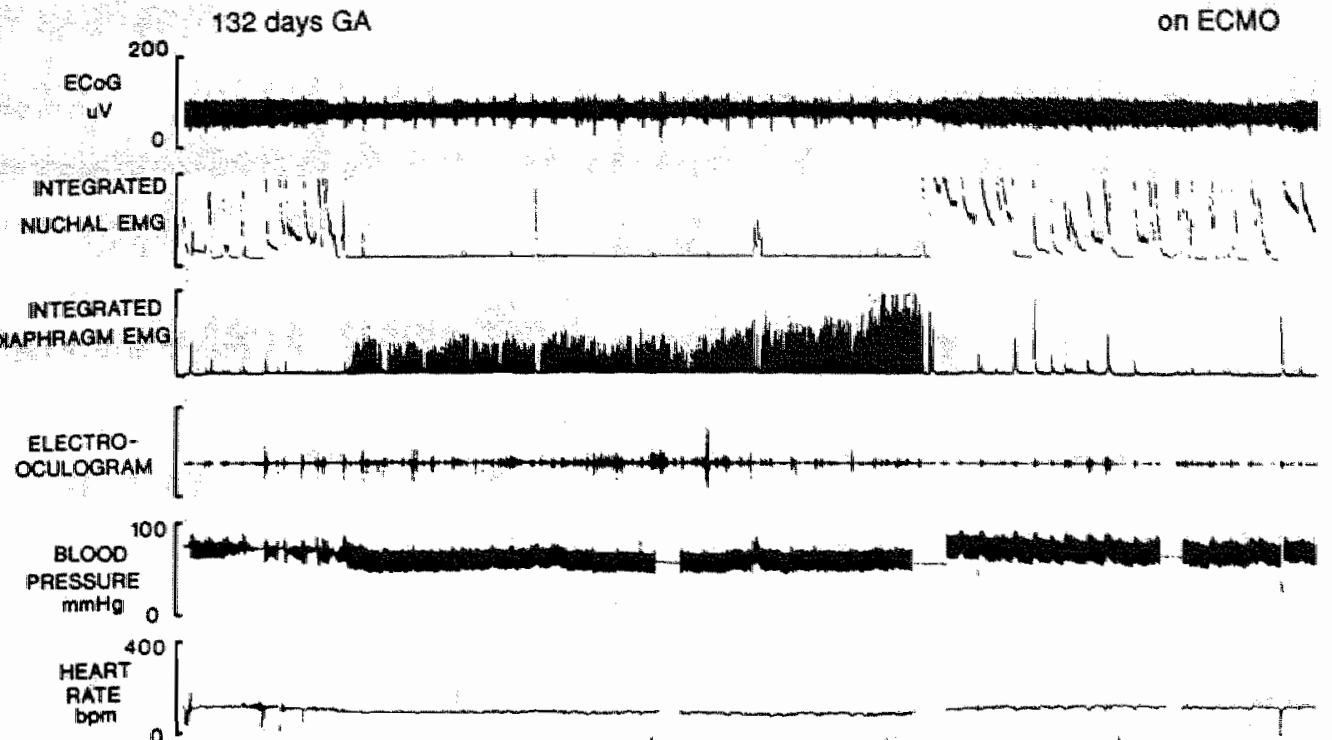

Normocapnia

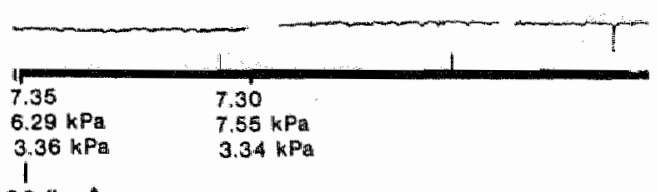

Hypercapnia

10 minuta:

Intrauterine recording of a fetus at 132 days gestation, 3 days after surgery. Tracings are from the top: electrocortical activity, integrated nuchal EMG, integrated diaphragm EMG. electro oculogram, blood pressure and heart rate. Blood gas and pH samples were taken at the indicated times, $\mathrm{CO}_{2}$-llow was increased at the indicated time. Note thath lotid

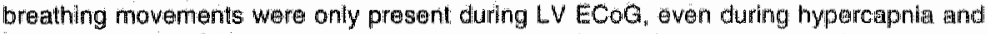
asscciated with rapid eye mowenents. Nuchal muscie activity was present during HV ECOG.

\section{Hypercapnia \& central cooling on ECMO}

In 5 out of 7 experiments fetal $\mathrm{PaCO}_{2}$ did not increase significantly after 20-30 min while fetal central temperature was clecreased by 1 "C. Fetal breathing remained present only during $L V$ ECOG despite fetal central temperature being decreased by $1{ }^{\circ} \mathrm{C}$. In these experiments fetal bypercapnia was obtained by increasing $\mathrm{CO}_{2}$ - 1 low to the membrane lung. In 2 out of 7 experiments fetal $\mathrm{PaCO}_{z}$ increased to a hypercapnic level without increasing $\mathrm{CO}_{2}$-flow to the membrane lung. 


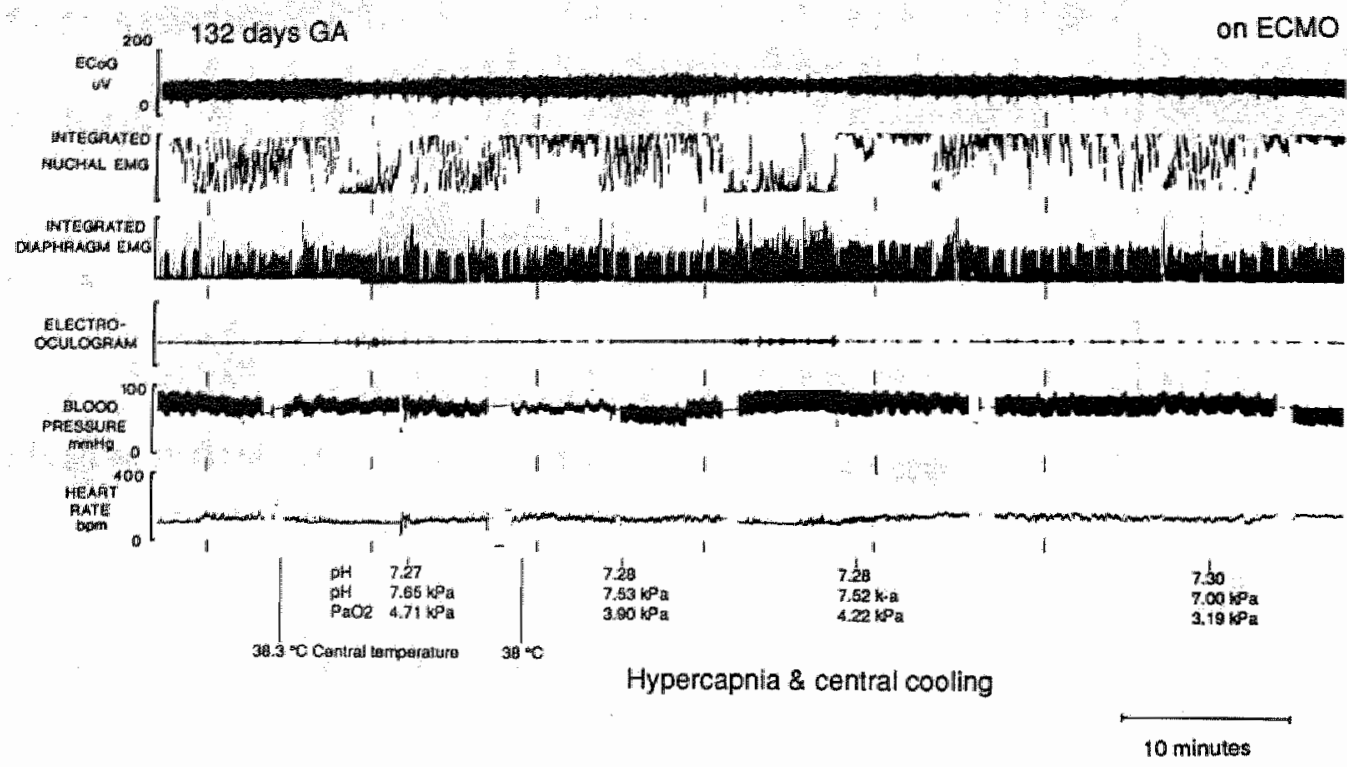

Figure 5.2

Intrauterine recording of a letus at 132 days gestation, 4 days after surgery. Tracings are from the top: electrocortical activity, integrated nuchal EMG, integrated dlaphragm EMG, electro oculogram, blood pressure and heart rate. Blood gas and pH samples were taken at the indicated times. Note that fetal breathing movements were still present during HV ECoG. Nurchal muscle actiwly was contimuousiy present. Both were still modulated by eledrocortical activity.

In the experiments where hypercapnia was associated with central cooling, central temperature had decreased by a mean of $2.1^{\circ} \mathrm{C}$ and fetal $\mathrm{PaCO}_{2}$ increased to $7.90 \pm 0.13 \mathrm{kPa}$. Nuchal muscle activity was present during both $\mathrm{ECOG}$ states, increased during HV ECOG and still present during LV ECoG but decreased in amplitude (see figure 5.2). The amount of nuchal muscle activity was $191.0 \pm 30.6 \%$ ( $\mathrm{p}<0.05$ compared to hypercapnia on $\mathrm{ECMO}$ ). Rapid eye movements were present during LV ECOG. In 3 out of 7 fetal lambs breathing activity remained associated with $\mathrm{LV}$ ECoG. Breathing activity was present continuously in 4 out of 7 fetal lambs. In these experiments breathing movements were both present during HV ECoG and LV ECoG (see figure 5.2). During hypereapnia associated with central cooling frequency of fetal breathing mowements decreased significantly compared to hypercapnia alone. However, there was no change in amplitude and breathing movements were still modulated by electrocortical activity resulting in a lower frequency during $\mathrm{HV}$ ECoG than during $\mathrm{LV}$ ECoG. 


\section{Discussion}

The incidence, frequency and amplitude of fetal breathing movements during LV ECoG increased significanty during hypercapnia as expected (Boddy et al. 1974). There was no change in incidence or length of periods of LV ECOG either during hypercapnia or during hypercapnia associated with central cooling. During hypercapnia associated with central coolling there was an increase in nuchal muscle activity, blood pressure, heart rate, a decrease in frequency of fetal breathing movements but no change in amplitude. Furthermore, breathing activity became continuous in 4 out of 7 fetal lambs. These results showed that the association of hypercapnia with central cooling could override the normally present inhibition of fetal breathing movements during HV ECoG resulting in continuous breathing activity.

As previously discussed ECMO could introduce new variables which could change fetal behavior. However, during ECMO there was normal fetal behaviour expressed by a normal incidence of LV ECoG and fetal

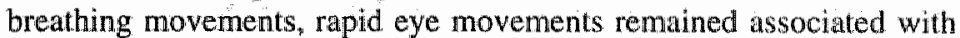
LV ECOG and nuchal muscle activity with $\mathrm{HV}$ ECoG. It is therefore unlikely that the use of extracorporeal circulation by itself could have influenced the results. The originality of our work was that we could control fetal temperature and blood gases directly independently of the ewe or the placenta.

In utero, after maturation of electrocortical activity fetal breathing movements and rapid eye movements are only present during $L V E C O G$ and nuchal EMG activity is present during HV ECOG and absent during LV ECoG (Dawes et al. 1972, Clewlow et al. 1983, Ioffe et al. 1987). Hypercapnia is known to stimulate fetal breathing movements (Boddy et al. 1974, Chapman et al. 1980, Ritchie \& Lakhani 1980, Bowes et al. 1981 b, Dawes et al 1982, Jansen et al. 1982, Clewlow et al. 1983, Rigatto et al. 1988). Fetal hypercapnia obtained with ECMO produced an increase in frequency, amplitude and incidence of fetal breathing movements during LV ECoG which is in agreement with earlier studies. During these hypercapnia periods there was no change in fetal behavioral activity, since there was no change in incidence or length of periods of $\mathrm{J}_{\text {w }} \mathrm{V} \mathrm{ECOG}$, eye movements remained associated with LV ECOG and muchal EMG with HV ECoG. At variance with previous reported experiments an increase in incidence of $L V$ ECoG during fetal hypercapnia obtained by ECMO did not occur. The increase in incidence of $\mathrm{LV}$ ECOG during maternal hypercapnia could be due to the release of substances produced by the placenta or the ewe during maternal hypercapnia. During hypercapnia associated with central cooling there was an increase in amplitude compared to control and a decrease in frequency. The decrease in frequency might be related to the decrease in oxygen consumption during cooling periods. Breathing movements were still modulated by electrocortical activity since the frequency of breathing movements was lower during HV ECOG than during LV ECoG. This might be due to the difference of control during $L V$ ECOG and during HV ECOG since 
breathing movements are metabolically controlled during HV ECoC, behaviorally controlled during LV ECoG (Phillipson et all. 1986).

Hypercapnia alone was not sufficient to initiate respiratory activity during HV BCoG, as reported earlier (Walker et al. 1976, Dawes et al. 1982, Jansen et al 1982, Rigatto et al 1988, Faucher et al. 1991). The mechanism for this inhibition during $\mathrm{HV} E \mathrm{ECO}$ is not very well understood. In utero breathing activity seems to be much more dependent on peripheral unput during HV ECoG and this mechanism could be involved in fetal apnea during HV ECoG. This was supported by experiments where continuous breathing activity was induced by electrical stimulation of the sciatic nerve (Moss \& Scarpelli 1979) or by decreasing cutaneous fetal temperature (Gluckman et al. 1983). Central mechanisms also play a role since fetuses with a brain stem transection or with small lesions in the rostral lateral pons can show fetal breathing movements during HV ECoG (Dawes al. 1983, Johnston \& Gluckman 1989). In the latter, continuous breathing only occurred during hypercapnia which suggested that the lesions interfered with cortical inhibitory influences changing the sensitivity for $\mathrm{CO}_{2}$ within the brain stem. In our experiments in 4 out of 7 fetuses breathing activity became present continuously when hypercapnia was associated with central cooling. This suggests that extra peripheral afferent input produced by central cooling might have changed central inhibitory processes allowing an increase in the sensitivity for $\mathrm{CO}_{2}$. We cannot explain why breathing did not become continuously in all experiments, since there was no difference in the decrease of central temperature, in the amount of nuchal muscle activity or in the lewel of fetall $\mathrm{PaCO}_{2}$. It could be speculated that the presence of continuous breathing is due to subtle changes in $\mathrm{CO}_{2}$ sensitivity produced by increased afferent input. The amount of afferent input was in this work not quantified more than by measuring the amount of nuchal muscle activity and temperature changes. Therefore, it is impossible to know whether the stimulus was similar. The quantification of afferent input is difficult and it would require direct recording of brain stem reticular substance. Subtle changes in $\mathbb{E C o G}$ or states were also not possible to observe due to the characteristics of our recording technique. Previous attempts to induce continuous breathing in utero by decreasing central temperature with an internal coil passing the pharynx, the larynx, oesophagus, and a loop in the stomach, were unsuccessful (Gluckman et al. 1983). It is possible that upper airway receptors were stimulated by the low temperature of the coil passing the upper airway and inhibited breathing activity. Another problem could have been that in those experiments the fetuses remained normocapnic. In our experiments breathing activity remained absent during $\mathrm{HV} \mathrm{ECOG}$ when fetal $\mathrm{PaCO}_{2}$ did not increase during central cooling. Further indication that fetal continuous breathing might be determined by the level of fetal $\mathrm{PaCO}_{2}$ is given by experiments where continuous breathing produced by hypercapnia associated with central cooling was not sustained when the fetal $\mathrm{PaCO}_{2}$ was decreased to normocapnic or hypocapnic level before birth (Kuipers et al. 1994c). In our study the association between central cooling and hypercapnia was essential for producing continuous breathing 
activity since neither could produce it independently. Further evidence of the influence of temperature on $\mathrm{CO}_{2}$ sensitivity was provided by experiments done on newborn animals which showed a decrneased respiratory response to $\mathrm{CO}_{2}$ in a warmer environment (Wantabe et al. 1993, Malcolm \& Henderson-Smart 1994).

Central hypothermia did not change the fetal ECoG pattern. Nuchal muscle activity was increased but still modulated by $L V$ ECOG. We assumed that this increased activity of the nuchal muscles reflected shivering activity since this enhanced activity was only present during the cooling periods (Gluckman et al. 1983).

There was a significant increase in blood piessure and heart rate during hypercapnia associated with central cooling, probably due to an incretse of catecholamine levels (Sidi et al, 1983, Gunn et al. 1985).

In summary, direct fetal hypercapnia resulted in a significant increase in amplitude, frequency and incidence of fetal breathing movements during LV ECoG but no increase in incidence of LV ECoG. Central cooling associated with hypercapnia could override the inhibition during HV ECoG resulting in continuous breathing. It might be speculated that cooling could have increased afferent input to the brain stem changing the $\mathrm{CO}_{2}$ sensitivity and overriding the inhibition during $\mathrm{HVECOG}$. This mechanism could contribute to the initiation of continuous breathing at birth. 


\section{Chapter 6}

\section{The effect of maternal hypoxemia on behavior in unanesthetized normoxic or mildly hyperoxic fetal lambs}

I.M. Kuipers, W.J. Maertzdort, H. Keunen, D.S. de Jong', M.A. Hanson ${ }^{2}$ and C.E. Blanco.

Dept. of Neonatology, Dept. of Cardiothoracic Surgery \& Dept. of Extra Corporeal Circulation', Acrademic Hospital Maastricht, University of Limburg, Maastricht, the Netherlands, Dept. of Obstetrics \& Gynaecology", University College London, London, United Kingdom.

J. Appl. Physiol. 76: 2535-2540, 1994. 


\section{Abstract}

To determine if hypoxemia inhibits fetal activity by substances from the mother or placenta, six fetal lambs were chronically instrumented at 128-132 days gestation for $\mathrm{ECMO}$. Severe maternal hypoxemia $\left(\mathrm{PaO}_{2}\right.$ decreased to $6.00 \pm 0.60 \mathrm{kPa}$ ) was produced whille fetal $\mathrm{PaO}_{2}$ was maintained normoxic or mildly hyperoxic using ECMO. The incidence of fetal breathing movements was $34.8 \pm 3.1 \%$ during baseline before $\mathrm{ECMO}, 36.8 \pm 3.4 \%$ during baseline on $\mathrm{ECMO}$, and $21.4 \pm 3.5 \%$ ( $p<0.05$ compared to baseline on ECMO) during maternal hypoxemia. The duration of periods of breathing was $9.8 \pm 1.2 \mathrm{~min}$ before ECMO, $9.3 \pm 1.1 \mathrm{~min}$ on ECMO and $10.5 \pm 1.7 \mathrm{~min}(\mathrm{~ns})$ during maternal hypoxemia. In 7 of 14 maternal hypoxemia experiments breathing activity stopped too late $(7-23 \mathrm{~min})$ to be attributed to maternal hypoxemia. Fetal ECoG activity (ns), nuchal muscle activity (ns) and rapid eye movements were present as normal before and on ECMO and during maternal hypoxemia and fetal blood pressure or heart rate did not change.

We conclude that the inhibition of fetal activity during maternal hypoxemia does not seem to be mediated by release of factors from the maternal side of the placenta or the ewe.

\section{Introduction}

In contrast to after birth acute hypoxemia inhibits fetal activity. This is seen as a decrease in breathing activity (Blanco et al. 1983b, Bocking et al. 1988, Boddy et al. 1974, Koos et al. 1987a), in limb movements and nuchal muscle activity (Blanco et al. 1983b, Bocking et al 1988, Koos et al. $1987 \mathrm{a}$, Natale et al. 1981, Woudstra et al. 1990), in rapid eye movements (Bocking et al. 1988, Koos et al. 1987a), in incidence and frequency of swallowing (Sherman et al. 1991) and in the magnitude of hind-limb reflexes (Blanco et al 1983b). The length of periods and incidence of $\mathrm{ECOG}$ activity may or may not be affected by acute hypoxemia (Adamson et al. 1984, Boddy et al. 1974). The inhibitory response to hypoxemia occurs rapidly: for example, fetal breathing movements are inhibited within 4 min (Clewlow et al. 1983, Koos et al. 1987a), hind-limb spinal reflexes are reduced within 5 min (Blanco et al. 1983b). Cardiovascular responses, seen as the increase in arterial blood pressure and the decrease in heart rate, occur within $3-4$ min (Blanco et al. $1983 \mathrm{~b}$ ).

Several studies have implicated an area in the upper pons of the fetal or neonatal brainstem in mediating the fetal response to hypoxemia. These studies employed a range of techniques, including brainstem transection (Dawes et al. 1983), focal cooling (Moore et all. 1991), the placement of lesions in the lateral pons (Gluckman \& Johnston 1987), electrical stimulation or recording from neurons in the lateral pons (Coles et al. 1989). These studies have been interpretated as showing that an active 
central inhibition is triggered by a decrease in brain tissue oxygenation. However, the mechanisms involved in this response and the nature and location of the chemosensitive area which senses the change in oxygen awailability are unknown.

In the majority of the studies the effects of short term acute hypoxemia on fetal activity were examined. Fetal hypoxemia was induced by having the ewe breathe a hypoxic gas mixture or by reducing uterine blood flow (Bocking Harding 1986, Boddy et al. 1974). Alternatively, a reduction in $\mathrm{O}_{2}$ availibility by means of fetal methemoglobinemia, fetal carboxyhemoglobinemia or fetal anemia has also been found to produce cessation of fetall breathing activity (Koos et al. 1988b, Koos et al. 1990a, Koos et al. 1987b). These studies show that a fall in tissue oxygenation, rather than a decrease in $\mathrm{PaO}_{2}$, is responsible for producing the cessation of fetal breathing activity. However the site at which this fall in oxygenation is detected is not apparent from these studies. For example, they cannot rulle out the possibility that release of modulatory substances from the fetal side of the placenta played a part in mediating the response. Indeed, it is known that $\mathrm{O}_{2}$-deficient tissues, such as the brain, heart and placenta produce substances (e.g. prostaglandins, adenosine and beta-endorphins) which could modulate fetal activity (Kitterman et al. 1983, Koos et al 1992, Slegel et al. 1988). These substances could mediate their effects via the lateral pons, alternatively, it may be that the lateral pons must be functionally intact for their effects to be seen. This would explain the observations that destruction of the brain stem alters the response (Koos et al. 1992).

This paper presents the results of studies aimed at examining the possibility that a substance produced by the mother or the maternal side of the placenta during hypoxemia produces an inhibitory effect on fetal behavior. In order to approach this problem we used an ECMO system in chronically instrumented fetal lambs. This allowed us to keep the fetus normoxic or mildly hyperoxic while the ewe was exposed to acute hypoxemia by breathing a hypoxic gas mixture.

\section{Materials and methods}

Experiments were performed on unanesthetized chronically instrumented fetal lambs in utero (Chapter 3).

Fetal blood gases and $\mathrm{pH}$, the incidence per hour and the length of periods of $L V E C O G$ activity, the incidence of nuchal muscle activity during HW ECoG, the incidence per hour and length of periods of fetal breathing movements were measured a) during periods before connection to the ECMO system, b) during baseline periods on ECMO and c) when the ewe was exposed to acute hypoxemia and the fetus remained normoxic or mildly hyperoxic. Maternal hypoxemia was obtained by having the ewe breathe through a bag supplied with a gas mixture of $9 \% \mathrm{O}_{2}, 3 \% \mathrm{CO}_{2}$ and $88 \% \mathrm{~N}_{2}(40 \mathrm{l} / \mathrm{min})$. During this time the fetus was kept normoxic or mildly byperoxic using the ECMO system. Maternal hypoxemia was initiated when the fetus showed breathing activity for at 
least one minute. The incidence of fetal breathing movenents and LV ECoG ( $\mathrm{min} / \mathrm{hr}$ ) was only analyzed for experiments with a duration of more than $30 \mathrm{~min}$. Mean blood pressure and heart rate were measured every $10 \mathrm{~min}$ during baseline recordings before connection to the ECMO system, during baseline recordings on ECMO and every 5 min during maternal hypoxemia experiments. Amniotic pressure was not subtracted from the blood pressure.

The Friedman and Wilcoxon signed rank test were used for statistical comparison of the incidence of nuchal EMG activity during $\mathrm{HV} E \mathrm{ECO}$, the incidence and length of periods of $L V E C O G$, the incidence and length of periods of fetal breathing movements, blood pressure and heart rate between baseline periods before connection to the ECMO system and the baseline periods on ECMO, and between the latter and periods of maternal hypoxemia. The median was used to express the time between experiments and the duration of maternal hypoxemia experiments.

\section{Results}

Experiments were performed on 6 fetal lambs, at 131-135 days gestational age.

\section{Baseline recordings before connecting the fetus to the ECMO system}

Baseline recordings were obtained 55 to 70 hours after the operation, the duration of these recordings being 11 to 28 hours. Fetal blood gases and $\mathrm{pH}$ just before connection to the ECMO system were: $\mathrm{pH} 7.35 \pm 0.01$, $\mathrm{PaCO}_{2} 5.92 \pm 0.20 \mathrm{kPa}, \mathrm{PaO}_{2} 2.28 \pm 0.22 \mathrm{kPa}$. Mean arterial blood pressure was $66 \pm 4 \mathrm{mmHg}$ (unreferenced), heart rate was $157 \pm 10 \mathrm{bpm}$.

Nuchal EMG activity was present $81.2 \pm 5.7 \%$ of the time during HV ECoG activity. Rapid eye movements ( 2 fetuses) were associated with LV ECoG activity and fetal breathing movements. Fetal breathing movements were only present during LV ECoG. LV ECoG activity occurred $52.5 \pm 2.0 \%$ of the time and the mean length of the LV ECoG periods was $13.5 \pm 0.8 \mathrm{~min}$ (table 6.1). Fetal breathing activity was present $34.8 \pm 3.1 \%$ of the time, the mean length of the fetal breathing periods being $9.8 \pm 1.2$ min (table 6.1 ). 
Incidence and length of periods of fetal breathing movements and LV ECOG activity during baseline recordings before connection to the ECMO system, during baseline recordings after connection to the ECMO system and cluring maternal hypoxemia experiments..

\begin{tabular}{llll}
\hline $\begin{array}{l}\text { Physiologic } \\
\text { variables }\end{array}$ & $\begin{array}{l}\text { Baseline } \\
\text { before ECMO }\end{array}$ & $\begin{array}{l}\text { Baseline } \\
\text { on ECMO }\end{array}$ & $\begin{array}{l}\text { Maternal } \\
\text { hypoxemia }\end{array}$ \\
\hline LV ECOG (\%) & $52.5 \pm 2.0$ & $47.5 \pm 2.0$ & $44.3 \pm 7.4$ \\
LV ECOG, LP (min) & $13.5 \pm 0.8$ & $14.3 \pm 1.5$ & $17.4 \pm 2.3$ \\
FBM (\%) & $34.8 \pm 3.1$ & $36.8 \pm 3.4$ & $21.4 \pm 3.5^{\%}$ \\
FBM, LP (min) & $9.8 \pm 1.2$ & $9.3 \pm 1.1$ & $10.5 \pm 1.7$
\end{tabular}

Means \pm SEM of incidence and length of periods of LV ECoG and fetal breathing mowements. (LP; length of period, FBM; fetal breathing mowements, \%; percentage of the time present, "p<0.05, compared to baseline on ECMO).

\section{Baseline recordings after connecting the fetus to the ECMO system}

On the third day post-surgery the fetuses were connected to the ECMO system. Baseline recordings were obtained in the different fetuses from one hour after connection to the ECMO system. Fetal pH and blood gases during these baseline recordings on the ECMO system were: $\mathrm{pH} 7.37 \pm 0.02, \mathrm{PaCO}_{2} 6.16 \pm 0.18 \mathrm{kPa}, \mathrm{PaO}_{2} 3.92 \pm 0.36 \mathrm{kPa}\left(\mathrm{PaO}_{2}\right.$, $\mathrm{p}<0.05$ compared to before connection to the ECMO system). Mean arterial blood pressure was $74 \pm 5 \mathrm{mmHg}_{\text {n }}$ heart rate was $171 \pm 13 \mathrm{bpm}$. Nuchal EMG activity was present $88.2 \pm 4.8 \%$ of the time during HV ECoG activity. Rapid eye movements $(n=2)$ were always associated with LV ECoG activity and fetal breathing movements. Fetal breathing movements were only present during LV ECoG. The incidence of LV ECoG activity was $47.5 \pm 2.0 \%$ and the mean llength of the LV ECoG periods was $14.3 \pm 1.5 \mathrm{~min}$ (table 6.1 ). Breathing activity was present $36.8 \pm 3.4 \%$ of the time, the mean length of the fetal breathing periods being $9.3 \pm 1.1 \mathrm{~min}$ (lable 6.1 ). 
Fetal and maternal bllood gases and $\mathrm{pH}$ before, at $5 \mathrm{~min}$ and at $30 \mathrm{~min}$ of maternal hypoxemia experiments.

\begin{tabular}{llll}
\hline & before $M H$ & $5 \mathrm{MH}$ & $30^{\prime} \mathrm{MH}$ \\
\hline $\mathrm{F}-\mathrm{pH}$ & $7.36 \pm 0.01$ & $7.36 \pm 0.01$ & $7.36 \pm 0.01$ \\
$\mathrm{~F}-\mathrm{PaCO}_{2}(\mathrm{kPa})$ & $6.20 \pm 0.22$ & $6.18 \pm 0.18$ & $5.90 \pm 0.35$ \\
$\mathrm{~F}-\mathrm{PaO}_{2}(\mathrm{kPa})$ & $5.37 \pm 0.54$ & $4.18 \pm 0.47^{7}$ & $4.36 \pm 0.55$ \\
$\mathrm{M}-\mathrm{pH}$ & $7.49 \pm 0.01$ & $7.50 \pm 0.03$ & $7.50 \pm 0.03$ \\
$\mathrm{M}-\mathrm{PaCO}_{2}(\mathrm{kPa})$ & $3.98 \pm 0.20$ & $4.03 \pm 0.19$ & $3.96 \pm 0.18$ \\
$\mathrm{M}-\mathrm{PaO}_{2}(\mathrm{kPa})$ & $13.58 \pm 0.70$ & $6.00 \pm 0.60^{1}$ & $5.63 \pm 0.77^{\dagger}$ \\
\hline
\end{tabular}

Means \pm SEM of fetal (F) en maternal (M) blood gases and $\mathrm{pH}$. ( $1 \mathrm{p}<0.05$, compared to measurement before maternal hypoxemita (MH) experiments).

\section{Maternal hypoxemia experiments}

A total of 14 maternal hypoxemia experiments were performed on 6 fetuses. One experiment is shown in figure 6.1. The experiments were carried out from 1 hour to 51 hours after connecting the fetal lamb to the ECMO system. The time between experiments ranged from $11.5 \mathrm{~min}$ to 18 hours $49.5 \mathrm{~min}$ (median $51.5 \mathrm{~min}$ ). Once again the periods were determined at random. The duration of the maternal hypoxemiat experiments ranged from $5 \mathrm{~min}$ to 1 hour $16 \mathrm{~min}$ (median $31 \mathrm{~min}$ ). The duration of experiments in which the incidence of fetal breathing movements $(n=5$, exp=8) and LV ECoG activity $(n=5$, exp=8) was measured was $45.0 \pm 6.4 \mathrm{~min}$. Maternal and fetal blood gases before and during the maternal hypoxemia experiments are shown in table 6.2. Felal $\mathrm{pH}$ and $\mathrm{PaCO}_{2}$ remained constant throughout the experiments; fetal $\mathrm{PaO}_{2}$ was slightly higher $(5.37 \pm 0.47 \mathrm{kPa})$ than its mean value during control $(3.92 \pm 0.36 \mathrm{kPa})$, it then decreased to $4.18 \pm 0.47 \mathrm{kPa}$ in the first $5 \mathrm{~min}$ of the maternal hypoxemia $(p<0.05)$. This latter value is still in the normoxic fetal range (table 6.2). During the maternal hypoxemia experiments mean arterial blood pressure was $69 \pm 3 \mathrm{mmHg}$ and heart rate was $169 \pm 9 \mathrm{bpm}$.

Nuchal EMG activity was present $85.6 \pm 6.2 \%$ of the time during HV ECoG activity. Rapid eye movements $(n=2)$ were present during LV ECoG activity and were associated with fetal breathing movements. Fetal breathing movements were only present during LV ECOG. The incidence of LV ECoG activity was $44.3 \pm 7.4 \%$ and the mean length of the LV ECoG periods was $17.4 \pm 2.3 \mathrm{~min}$ (table 6.1). Breathing activity 


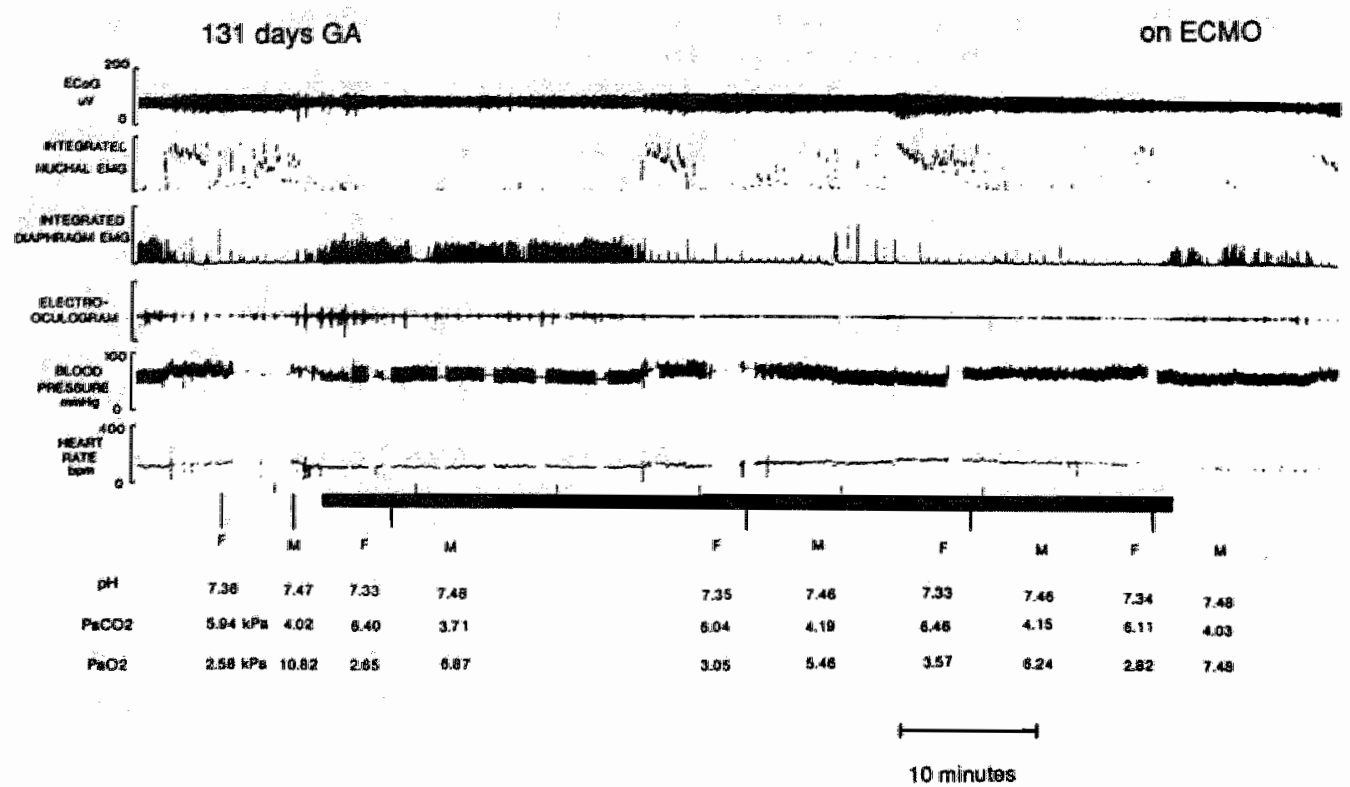

Figure 6.1

Intrauterine recording of a fetus of 131 days of gestation connected to the ECMO system 3 days after suigery. Tracings are from the top: ECOG activity, integrated nuchal $E M G$, integraled diaphragm EMG, electro-oculogram, blood pressure and heart rate. At the indicated times fetal (F) and matemal $(M)$ arterial blood samples were taken for blood gases and $\mathrm{pH}$. Breathing activity and rapid eye movements remain present during $\mathrm{LV}$ ECOG activity despite maternal hypoxemia (represented by the black bar). Nuchal EMG activity is present and associated with HV ECOG activity.

was present $21.4 \pm 3.5 \%$ of the time $(p<0.05$ compared to the incidence during baseline recordings on $\mathrm{ECMO}$ ) and the mean length of the fetal breathing periods was $10.5 \pm 1.7 \mathrm{~min}$ (table 6.1 ). Of the 14 experiments breathing activity stopped in 7 experiments within $7 \mathrm{~min}$; in 7 experiments it stopped between $7-23$ min after starting maternall hypoxemia. When fetal breathing stopped (within 7 min in 7 experiments) this was associated with a switch to HV ECoG and the occurrence of nuchal EMG activity. In only one experiment did fetal breathing stop in LV ECoG.

\section{Discussion}

Using an ECMO system made it possible to maintain fetal oxygenation while the maternal tissues and the maternal side of the placenta were exposed to hypoxemia. Apart from a modest decrease in the incidence of breathing activity, the well established fetal effect of acute maternal 
hypoxemia. e.g. a cessation of fetal breathing movements, did not occur despite the fact that we reduced maternal $\mathrm{PaO}_{4}$ to levels proviously reported to produce cessation of fetal breathing activity. This allows us to conclucle that the overall fetal response to hypoxemia previously described when asing maternal hypoxemia (Boddy et al. 1974) or utero-placental hypoxemia (Bocking \& Harding 1986) does not seem to be mediated by the release of substances produced by the ewe or the maternal side of the placenta.

The ECMO system itself could theoretically influence fetal behavioral activity. To control for this, we analyzed the incidence and duration of periods of fetal breathing movements and of LV ECOG activity before and after fetuses were connected to the ECMO system, while fetal blood gases and $\mathrm{pH}$ were held in the normal range. There was no statistical difference in the incidence or duration of periods of fetal breathing movements or LV ECoG activity before and on ECMO. Furthermore, after connection to the ECMO system, there was normal cycling of fetal states where nuchal EMG activity was associated with $\mathrm{HV}$ ECoG activity and rapid eye movements with LV ECoG activity. There was no statistical difference in blood pressure and heart rate before and on ECMO. Since there was no abnormal behavioral activity during the baseline periods on ECMO, it is unlikely that the use of extracorponeal circulation by itself influenced our results.

The effects of acute hypoxemia on fetal breathing activity induced by giving the ewe a hypoxic gas mixture to breathe or by decreasing uterine blood flow, have been analyzed in different ways. Investigators have described the immediate effect of acute hypoxemia on fetal breathing movements (Blanco et al 1983b, Boddy et al. 1974, Clewlow et al. 1983; Koos et al. 1987a) and on the length of periods of fetal breathing movements (Koos et al. 1987a), or they have described the sustained effect of hypoxemia on the incidence of fetal breathing movements per hour (Boddy et al. 1974, Koos et al. 1987a). ECoG activity was also analyzed in different ways (Adamson et al. 1984, Blanco et al. 1983b, Bocking et al. 1988, Bocking \& Harding 1986, Clewlow et al. 1983, Koos et al. 1987a) yielding contradictory results (Adamson et al. 1984, Bocking et al. 1988, Bocking \& Harding 1986. Boddy et al. 1974, Koos et al. 1987). Combining the findings from the diverse methods it can be concluded that hypoxemia produces an acute response within 7 min which is sustained for at least one hour. Therefore we chose to analyze the immediate effects on the fetus within 5 min of induction of acute maternal hypoxemia in terms of fetal breathing activity, the length of periods of fetal breathing movements, LV ECoG activity and cardiovascular responses. We then analyzed the sustained effects of hypoxemia by describing the incidence of fetal breathing movements, LV ECoG activity, and the presence of nuchal EMG activity and rapid eye movements over the whole period of hypoxemia. In this way we felt that we would obtain a more precise interpretation of the response.

After exposing the ewe to a hypoxic gas mixture, it takes $2.5 \times 5 \mathrm{~min}$ before the fetus becomes hypoxemic and shows a response (Blanco et al. 1983b, Clewlow et al. 1983, Koos et al. 1987a). Within 7 min of making 
the ewe hypoxemic (maternal $\mathrm{PaO}_{2}, 6.0 \pm 0.6 \mathrm{kPa}$ ) we expected to see a decrease in fetal breathing activity if this was due to the release of substances from maternal tissues or the maternal side of the placenta. In our experiments fetal breathing activity stopped within $7 \mathrm{~min}$ in only 7 of the 14 experiments and it did not appear to be a typical response to acute hypoxemia as described previously (Clewlow et al. 1983, Koos et all. 1987 a) because nuchal muscle activity and rapid eye movements were present. In the rest of the experiments fetal breathing activity stopped too late (between $7-23 \mathrm{~min}$ in 7 of the 14 experiments) to be the result of acute hypoxemia. If the release of substances of maternal or placental origin during hypoxemia were the mechanism responsible for fetal inhibition, this inhibition should have been manifest within 7 min. The fact that fetal breathing movements eventually stopped was to be expected, due to their periodic nature. Furthermore, in our experiments the length of periods of fetal breathing movements was not significantly shortened by exposing the ewe to hypoxemia as occurs during fetal hypoxemia (Koos et al. 1987a).

During our maternal hypoxemia experiments there was no significant evidence of a fetal cardiovascular response. Catecholamines are known to cross the placenta ( $\mathrm{Gu} \&$. Jones 1986), although the increase in fetal plasma catecholamines is thought most likely to be of fetal (Cohen et al. 1982) than of maternal (Kitterman et al. 1983) origin. It therefore appears that, as the fetus remained normoxic or mildly hyperoxic, fetal carotid chemoreceptors or the adrenal medulla were not stimulated (Blanco et al. 1984).

The incidence of fetal breathing movements is reported to decrease to levels of $0-10 \%$ when the ewe is exposed to severe hypoxemia $\left(\mathrm{PaO}_{2}\right.$ $<50 \mathrm{mmHg}$, approximately $6.6 \mathrm{kPa}$ )(Adamson et al. 1984 , Boddy et al. 1974, Koos et all, 1987a). In our experiments maternal $\mathrm{PaO}_{2}$ was $6.0 \pm 0.6 \mathrm{kPa}(45 \pm 5 \mathrm{mmHg})$ and the incidence of fetal breathing activity decreased significantly, but only to $21 \%$. This may have been due to the small fall in fetal $\mathrm{PaO}_{2}$, from mildly hyperoxic levels $(5.37 \pm 0.54 \mathrm{kPa})$ to the upper normoxic range $(4.18 \pm 0.47 \mathrm{kPa})$ : However, it is not expected that this small fall in fetal $\mathrm{PaO}_{2}$ would produce inhibition unless adaptation to the new level occurred rapidly. There is some indirect evidence which could suggest that this is the case (Matsuda et al. 1992), although the experimental conditions, the time-scale and design are very different. If the decrease in $\mathrm{PaO}_{2}$ played a role this would again suggest that $\mathrm{PaO}_{2}$ and not hormonal mediators were responsible for the well described response during maternal hypoxemia. Furthermore, the decrease in incidence of fetal breathing movements was not associated with a decrease in nuchal muscle activity and rapid eye movements or a change in ECOG in our experiments. Thus the mechanism normally involved in the effects of hypoxemia on the fetus (Blanco et al 1983b. Bocking \& Harding 1986, Koos et al. 1987. Natale et al. 1981, Woudstra et al. 1990) was not induced in our experiments. Our results do not support therefore the idea of delayed transfer of an inhibitor.

We are aware that our protocol would not permit us to rule out a possible role of substances produced by the fetal side of the placenta and 
the membranes during hypoxemia because in our experiments these tissues remained normoxic or mildly hyperoxic: However, the fetal $\mathrm{PaO}_{2}$ remained less than maternal $\mathrm{PaO}_{2}$, thus the normal direction of $\mathrm{O}_{2}$ transport from mother to fetus was preserved. The role of the maternal circulation in preserving placental function is underscored by the observation that the placenta and fettl membranes remain intact and functional after total fetectomy in monkeys and baboons (Albrecht et al. 1989, Albrecht \& Pepe 1985 Nathanielsz et al. 1992). In addition the release of any substance from the fetal side of the placenta or membranes during hypoxemia has not been reported. Whilst fetal membranes and fetal cotyledons contain high concentrations of prostaglandins (Kelleman et al. 1992, Olson ef al. 1986) and prostaglandins are known to reduce fetal breathing activity (Kitterman et al. 1983), it is reported that fetall prostaglandins do not increase during the first hour of acute hypoxemia (Akagi et al. 1990a, Akagi \& Challis 1990b). Therefore, a role of prostaglandins as mediator for the inhibitory effect of breathing activity during acute hypoxemia is unlikely. During hypoxemia the placenta releases adenosine (Slegel et al. 1988) but it seems that adenosine produced in the CNS during fetal hypoxemia might have a more important role in the inhibition of fetal activity (Koos et al. 1992). Furthermore, fetal breathing movements were also inhibited when the $\mathrm{O}_{2}$ availability was reduced by fetal methemoglobinemia, fetal carboxyhemoglobinemia or fetal anemia (Koos et al. 1988b, Koos et al. 1990a, Koos et al. 1987b). These results provide additional support for our hypothesis that the hypoxemic inhibition of fetal breathing is not initiated by the ewe or the maternal side of the placenta.

In conclusion, the well described fetal response obtained by exposing the ewe to acute hypoxemia $\left(\mathrm{PaO}_{2}-6 \mathrm{kPa}\right)$ was not seen when the fetus was held normoxic or mildly hyperoxic using ECMO. Therefore, the mechanism responsible for the inhibition of fetal activity during maternal hypoxemia does not seem to be mediated by the release of some inhibitory factors from the matemal side of the placenta or the ewe. 


\section{Chapter 7}

\section{Fetal breathing is not initiated after cord occlusion in the unanesthetized fetal lamb in utero}

I.M. Kuipers, W.J. Maertzdorf, H. Keunen, D.S. de Jong", M.A. Hanson", C.E. Blanco.

Dept. of Neonatology, Dept. of Cardiothoracic Surgery \& Dept. of Extra Corporeal Circulation', Academic Hospital Maastricht, University of Limburg, Maastricht, the Netherlands, Dept. of Obstetrics \& Gynaecology2', University College London, London, United Kingdom.

J. Dev. Physiol. 17: 233-240, 1992. 


\section{Abstract}

We investigated the role of cord occlusion in the initiation of breathing at birth using an extracorporeal membane oxygenator system to control fetal blood gases independently of the placenta in 12 chronically instrumented fetal lambs. In group IA $\left(\mathrm{n}=9\right.$, exp=12) $\mathrm{PaCO}_{2}$ was kept constant $(5.62 \pm 0.21$ to $5.70 \pm 0.23 \mathrm{kPa})$ during cord occlusion. Group $\mathrm{IB}(\mathrm{n}=$ ?, exp=8) were cord occlusion experiments from group IA in which no fetal breathing movements had occurred; $\mathrm{CO}_{2}$ flow to the membrane was increased and fetal $\mathrm{PaCO}_{2}$ rose significantly $(5.45 \pm 0.24$ to $8.27 \pm 0.56 \mathrm{kPa})$. In group II $\left(\mathrm{n}=7\right.$, exp=12) $\mathrm{PaCO}_{2}$ was allowed to increase from $5.98 \pm 0.24 \mathrm{kPa}$ to $8.09 \pm 0.48 \mathrm{kPa}$ after cord occlusion Within $5 \mathrm{~min}$ of cord occlusion, fetal breathing movements did not occur in 11 out of 12 experiments in group $1 \mathrm{~A}$ or in 11 out of 12 experiments in group II. In contrast in group IB breathing did occur in 5 out of 8 experiments. When they occurred, fetall breathing movements were always associated with LV ECoG. Our results do not stpport the hypothesis that the initiation of breathing within 5 min of birth is dependent on an inhibitory factor of placental origin. Furthermore these data suggest an association between the presence of breathing and a substantial rise in $\mathrm{PaCO}_{2}$.

\section{Introduction}

At birth pulmonary gas exchange must be established after clamping the umbilical cord. This can only happen when the lungs are expanded with air or oxygen and when there is a sufficient respiratory drive to produce and maintain respiratory efforts.

In utero, fetal breathing movements are episodic, being present only during periods when the fetus shows rapid eye movements and LV ECOC activity (Dawes et al. 1972). These fetal brealhing movements are stimulated by hypercapnia (Boddy et al. 1974, Bowes et al. 198 lb, Chapman et al. 1980, Dawes et al. 1982, Rigatto et al. 1988) and inhibited during acute hypoxemia (Boddy et al 1974). In contrast. after birth breathing becomes continuous throughout all behavioural states, it is stimulated by hypoxia and is regulated by metabolic requirements. The transition from episodic fetal breathing to continuous neonatal breathing occurs at a time when many other changes occur and it is therefore difficult to isolate any single factor as being responsible for the intitiation of breathing activity at birth.

The removal of the umbilical circulation has been proposed as a mechanism which initiates continuous breathing at birth. It is hypothesized (Adamson et al. 1987, Blanco et al. 1987b) that cord occlusion interrupts the supply of a factor of placental origin which prevents fetal breathing movements from becoming continuous in utero; thus cord occlusion permits the establishment of continuous breathing. 
Adamson er al. (1987) and Blanco et al. (1987b) used mechanical ventilation or continuous positive pressure to oxygenate the fetus in utero after clamping the cord. Breathing was initiated after cord clamping in these experiments, and it stopped abruptly when the cord acclusion was released. However, despite the use of mechanical yentilation or continuous positive pressure in these experiments fetal $\mathrm{PaCO}_{2}$ increased substantially. The reason for the initiation of continuous breathing after cord clamping in these experiments is thus not clear because the exclusion of a possible placental factor and hypercapnia occurred simultaneously. In order to clarify further the mechanism for the initiation of breathing at birth we have used an extracorporeal membrane oxygenation system to control fetal $\mathrm{PaCO}_{2}$ after cord clamping. This has allowed us to address the questions a) Does the exclusion of the umbilical circulation per se play a role in the initiation of breathing at birth, and $b$ ) Does a rise in $\mathrm{PaCO}_{2}$ play a role during this transition?

\section{Materials and methods}

Experiments were performed on unanesthetized chronically instrumented fetal sheep in utero (see Chapter 3 ).

Baseline data on incidence of fetal breathing activity and its relationship with $L V$ ECOG were obtained before connecting the fetus to the ECMO system and after connection to the ECMO system. The effect of cord clamping on the establishment of continuous breathing was evaluated in three groups. After cord occlusion in utero there will always be an increase in fetal $\mathrm{PaCO}_{2}$ since the placenta is excluded. Therefore in Group IA we decreased the $\mathrm{CO}_{2}$ flow to the membrane in order to compensate for this and to keep fetal $\mathrm{PaCO}_{2}$ constant. Group IB consisted of fetuses of group IA which did not show breathing activity after $5 \mathrm{~min}$ of cord occlusion; in these animals $\mathrm{PaCO}_{2}$ was raised by increasing the $\mathrm{CO}_{2}$ flow to the membrane oxygenator. In group II the $\mathrm{CO}_{2}$ flow to the membrane oxygenator was not changed, therefore fetal $\mathrm{PaCO}_{2}$ was allowed to increase after cord occlusion. Complete occlusion of the cord was confirmed in each fetus by stopping the pump of the ECMO system for about 15 seconds at the end of cord occlusion: this produced a transient bradycardia and rise in blood pressure which were reversed when the pump was restarted. Also we verified during surgery and after birth that the cord occluder could be inflated tightly around the umbilical cord and could not leak. After the experiments we clamped the cord, sacrificed the mother with an overdose of barbiturate, and delivered the fetus by caesarean section. Meanwhile the fetus was supported with the ECMO system and blood gases were kept constant. After cord occlusion 5 min were allowed for breathing to be initiaited. If it was not present after that period elapsed, we classified the experiments as negative with the respect to initiation of breathing.

Wilcoxon signed rank test was used for statistical comparison of blood gas values before and during cord occlusion and for statistical comparison of the heart rate before and after cord occlusion. 
Experiments were performed on 12 fetal lambs, at 13 l-135 days gestational age.

Six of the twelve ewes showed signs of uterine contractions on the intrauterine pressure record and were judged to be in early labour.

\section{Baseline recordings before connecting to the extracorporeal membrane oxygenation system}

The baseline recordings were obtained 55 to 70 hours after the operation. Six ewes were in labour: in those preparations fetal breathing activity was present $17.8 \pm 4.8 \%$ of the time and $L V E C O G$ activity occurred $39.3 \pm 6.8 \%$ of the time. In the other six ewes which were not in labour fetal breathing activity was present $27.3 \pm 4.5 \%$ of the time and LV ECoG activity occurred $51 \pm 2.6 \%$ of the time. Nuchal muscle activity was always associated with HV ECoG. Fetal blood gases prior to connection to ECMO system were: $\mathrm{pH} 7.35 \pm 0.03$, $\mathrm{PaCO}_{2} 6.14 \pm 0.24 \mathrm{kPa}, \mathrm{PaO}_{2} 2.18 \pm 0.17 \mathrm{kPa}$.

\section{Baseline recordings after connecting to the ECMO system}

On the third day post surgery the fetuses were connected to the ECMO system. In the fetuses not exposed to labour we analyzed the incidence of fetal breathing activity during periods of normal fetal blood gases ( $\mathrm{pH} 7.37 \pm 0.03, \mathrm{PaCO}_{2} 5.99 \pm 0.20 \mathrm{kPa}$ and $\mathrm{PaO}_{2} 4.20 \pm 0.39 \mathrm{kPa}$ ). These recordings were obtained in some animals from one hour after connection to the ECMO system $(n=3)$ and in the other three at different times between experiments before or after cord occlusion. These periods ranged from 120 to 692 min and were not meant to be used as control for the cord occlusion experiments but to control for possible deleterious effects of ECMO on normal fetal behaviour. Breathing activity was present $36.20 \pm 3.75 \%$ of the time, LV ECaG activity $49.20 \pm 3.37 \%$ of the time. Furthermore all 12 fetuses presented breathing movements when challenged with hypercapnia $(n=9)$ before cord occlusion experiments, or when they were delivered at the end of the experiments $(n=7)$. A total of 24 cord occlusion experiments were performed in the 12 fetuses.

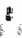

\section{Cord occlusion experiments}

The cord acclusion experiments in all groups were carried out 91 min to 24 hours after connecting the fetal lamb to the ECMO system. The time between cord occlusion experiments varied from 28 min to 12 hours and 5 min (mean $176 \pm 65 \mathrm{~min}$ ). The effect of cord clamping on the 
Table 7.1

Fetal $\mathrm{pH}$ and blood gases values just before and during cord acclusion experiments in all groups.

\begin{tabular}{|c|c|c|c|c|}
\hline 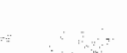 & & $p H$ & $\mathrm{PaCO}_{2}(\mathrm{kPa})$ & $\mathrm{PaO}_{2}(\mathrm{kPa})$ \\
\hline Group IA & $\begin{array}{l}\text { before } \\
\text { during }\end{array}$ & $\begin{array}{l}7.37 \pm 0.02 \\
7.32 \pm 0.03^{9}\end{array}$ & $\begin{array}{l}5.62 \pm 0.21 \\
5.70 \pm 0.23\end{array}$ & $\begin{array}{l}3.80 \pm 0.27 \\
4.19 \pm 0.47\end{array}$ \\
\hline Group IB & $\begin{array}{l}\text { before } \\
\text { during }\end{array}$ & $\begin{array}{l}7.40 \pm 0.02 \\
7.20 \pm 0.03^{1}\end{array}$ & $\begin{array}{l}5.45 \pm 0.24 \\
8.27 \pm 0.56\end{array}$ & $\begin{array}{l}3.86 \pm 0.25 \\
6.28 \pm 1.36^{\prime}\end{array}$ \\
\hline Group II & $\begin{array}{l}\text { before } \\
\text { during }\end{array}$ & $\begin{array}{l}7.37 \pm 0.01 \\
7.27 \pm 0.02^{\prime}\end{array}$ & $\begin{array}{l}5.98 \pm 0.24 \\
8.09 \pm 0.48^{:}\end{array}$ & $\begin{array}{l}6.27 \pm 0.80 \\
5.76 \pm 1.07\end{array}$ \\
\hline
\end{tabular}

Means \pm SEM of fetal blood gases and $\mathrm{pH} .(\mathrm{p} \leq 0.05$, compared to values betore cord occluston).

initiation of breathing was evaluated in three groups of fetuses. Fetal blood gases on extracorporeal membrane oxygenation system before and during the cord occlusion experiments are shown in table 7.1 .

When the cord was occluded there was an increase of $5 \pm 1.73 \mathrm{mmHg}$ in the arterial blood pressure which returned to control within $3 \mathrm{~min}$. Heart rate before cord occlusion was $162 \pm 6.8 \mathrm{bpm}$, and after cord occlusion it was $150 \pm 8 \mathrm{bpm}(\mathrm{p}=0.262)$. In 19 of the experiments fetal core temperature remained constant; in 3 experiments it decreased by $1.2{ }^{\circ} \mathrm{C}$, and in 2 it increased by $0.6{ }^{\circ} \mathrm{C}$. During cord occlusion nuchal muscle activity was associated with HV ECoG activity and not with LV ECoG activity.

\section{Group $I A$}

This group consisted of 9 fetuses (labour $n=5$ ) and 12 cord occlusion experiments (labour $\mathrm{n}=7$ ). By protocol the $\mathrm{PaCO}_{2}$ in this group was not allowed to increase significantly during cord occlusion (table 7.1). The duration of the cord occlusion experiments was $31 \pm 5.7 \mathrm{~min}$. At the moment of cord occlusion 8 fetuses were in HV ECoG activity; 3 of them switched to LV ECoG activity within 5 min. One fetus was in LV ECoG activity and switched to HV ECOG activity within $5 \mathrm{~min}$. In 3 experiments ECoG could not be analyzed. In 2 experiments the cord was occluded during a period of breathing activity. "This breathing activity had been present for 3 and $4.5 \mathrm{~min}$ respectively and it stopped 2 and $3.5 \mathrm{~min}$ after cord occlusion, coinciding with a swith to HV ECoG activity. These experiments were not considered positive. In group LA, fetal breathing movements did not occur in 11 of 12 experiments after cord occlusion. In one experiment breathing activity started $1 \mathrm{~min}$ after cord occlusion and electrocortical activity switched immediately to LV ECoG activity. Breathing activity was present during LV ECoG or intermediate electrocortical activity during this cord acclusion. This 
Initiation of fetal breathing movements within 5 min after cord occlusion in utero. Also shown are the percentage changes of fetal $\mathrm{PaCO}_{3}$ and the duration of the cord ocelusions.

\begin{tabular}{llll}
\hline & $\begin{array}{l}\text { Group } I A_{s} \\
n=9, \exp =12\end{array}$ & $\begin{array}{l}\text { Group } 1 B_{i} \\
n=7, \exp =18\end{array}$ & $\begin{array}{l}\text { Group } \|_{0}, \\
n=7, \exp =12\end{array}$ \\
\hline FBM present (all) & $1 / 12$ & $5 / 8$ & $1 / 12$ \\
FBM (laboul) & $1 / 7$ & $4 / 4$ & $0 / 5$ \\
FBM (non-labour) & $0 / 5$ & $1 / 4$ & $1 / 7$ \\
\% change in PaCO & $1.4 \pm 1.4$ & $52 \pm 8.4$ & $35 \pm 5.3$ \\
$\begin{array}{l}\text { Duration of } \\
\text { cord occlusions (min) }\end{array}$ & $31 \pm 5.7$ & $23.6 \pm 4.8$ & $31.3 \pm 3.2$
\end{tabular}

Presence of fetal breathing movements within 5 min after cord occlusion in utero. Means \pm SEM of the percentage changes of fetal $\mathrm{PaCO}_{2}$ and the dutation of cord occiusions. (FBM; fetal breathing movements; $\%$; percentage change of $\mathrm{PaCO}_{2}$ oxp; experiments:.

experiment was considered positive. Therefore the initiation of fetal breathing movements after cord occlusion occurred in 1 out of 12 experiments.

\section{Group IB}

This group consisted of 7 fetuses (labour $n=3$ ) and 8 of the 10 cord occlusion experiments (labour $n=4$ ) of group IA in which no breathing activity occurred. In these experiments extra $\mathrm{CO}_{2}$ was added to the ECMO system following protocol, and the $\mathrm{PaCO}_{2}$ for this group increased from $5.45 \pm 0.24 \mathrm{kPa}$ to $8.27 \pm 0.56$ (table 7.1 ). $\mathrm{PaO}_{2}$ also increased from $3.86 \pm 0.24 \mathrm{kPa}$ to $6.28 \pm 1.36 \mathrm{kPa}(\mathrm{p}=0.05)$ when the $\mathrm{CO}_{2}$ flow to the membrane oxygenator was increased. The duration of these experiments was $23.6 \pm 4.8$ min. In 5 experiments the fetuses were in HV ECoG at the moment of adding $\mathrm{CO}_{2}$ to the system. One remained in HV ECoG over the next $5 \mathrm{~min}$, the other 4 switched to LV ECoG within 5 min; in 3 experiments ECoC activity could not be amalyzed. Breathing activity occurred in 5 out of 8 cord occlusions (table 7.2). When present, fetal breathing movements were always associated with $L V$ ECoG. All fetuses not exposed to labour presented fetal breathing movements during cord occlusion and hypercapnia despite that the change in $\mathrm{PaCO}_{2}$ was not significantly different (labour group $52 \pm 10 \%$, non-labour group $51.85 \pm \mathbb{1 5 . 1 \% )}$. 


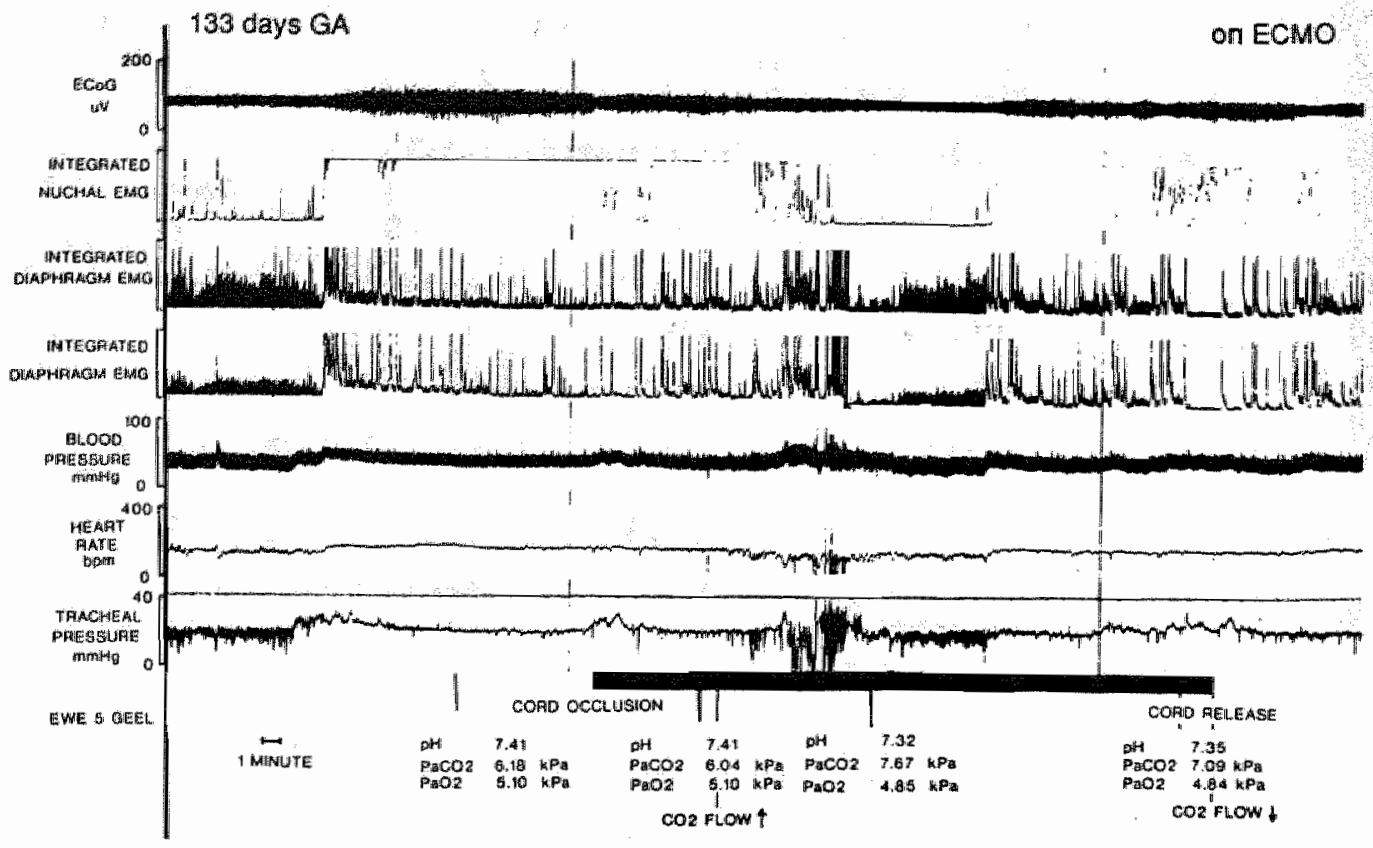

Figure 7.1

A recording from a fetus from Group IA, in utero, at 133 days gestation. Tracings are from the top: ECOG activity, integrated nuchal EMG, integrated diaphragmatic EMG (2 tracings), blood pressure, heart rate and tracheal pressure. At the indicated times the cord was occluded and released (represented by black bar), $\mathrm{CO}_{2}$ flow to the membrane was increased or decreased, and fetal blood gases and $\mathrm{pH}$ samples were taken. Breathing movements were present before cord acclusion. In the first 6 min after cord occlusion $\mathrm{PaO}_{2}$ remained constant and there was mo breathing activity present. After increasing $\mathrm{CO}_{2}$ llow to the membrane (Group (B) breathing movements were present during LV ECoG activity.

\section{Group II}

This group consisted of 7 fetuses (labour $n=3$ ) and 1.2 cord occlusion experiments (labour $n=5$ ). Fetal $\mathrm{PaCO}_{2}$ increased from $5.98 \pm 0.24 \mathrm{kPa}$ to $8.09 \pm 0.48 \mathrm{kPa}$ during cord occlusion for this group (table 7.1). The duration of the cord occlusion experiments was $31.3 \pm 3.08$ min. At the moment of cord occlusion, the fetuses were in HV ECOG in

4 experiments and they remained in this state in the experiments the first 5 min of cord occlusion; in 3 experiments they switched from HV ECOG to LV ECoG within $5 \mathrm{~min}^{*}$ in 2 experiments the fetuses were in LV ECoG and switched to HV ECoG within $5 \mathrm{~min}$; in 2 experiments the fetuses were in LV ECoG and remained in this state for the first $5 \mathrm{~min}$. In one experiment ECoG could not be analyzed. Breathing started after cord occlusion in only one experiment in this group: this was 4 min after cord occlusion and the fetus was in LV ECoG and not in labour. In one experiment the cord was occluded during a period of fetal breathing activity. Breathing was already present for $28 \mathrm{~min}$, and it stopped $3.5 \mathrm{~min}$ 
after cord occlusion. This was associated with a switch to HV ECOG activity. This experiment was considered negative.

\section{Discussion}

Our experiments were not designed to compare the incidence of fetal breathing movements before and after cord occlusion, but merely to examine whether or not cord occlusion by itself played a role in the onset of breathing. Thus we reasoned that if the exclusion of the placental circulation was the key component in the initiation of breathing at birth, then it should be initiated, after a labour period, within 60 to 90 seconds of clamping the cord (Mortola et al. 1982, Vyas al 1981). This should occur even if the fetus remained in utero and blood gases and core temperature were prevented from changing. Such breathing should occur regardless of the fetal ECoG state and whether the fetus was already showing fetal breathing movements or not due to the occurrence of labour. The experiments reported in this paper showed that breathing activity in utero was only established on $8 \%$ of occasions within 5 min after umbilical cord occlusion in fetal lambs supported with an ECMO system to prevent changes in fetal $\mathrm{PaCO}_{2}$. This was irrespective of whether or not these ewes were in labour. When fetal breathing movements did occur, they were always associated with LV ECoG.

In order to control for the theoretically influence of an extracorporeal circuit in fetal behaviour we analyzed the incidence of letal breathing movements during ECMO with normal fetal blood gases. We did not find a normal incidence of fetal breathing movements in our 6 animals in labour due to the inhibitory effects of labour on fetal breathing activity (Richardson et al. 1979, Kanaan et al. 1991 Molteni et al. 1980). However, we did find an normal incidence of fetal breathing activity during ECMO during periods of normal fetal blood gases in five fetuses not exposed to labour $(36.20 \pm 3.75 \%)$ which is similar to the incidence reported by other investigators (Boddy et al. 1974). Furthermore, in every fetus breathing could be stimulated by hypercapnia before cord occlusion or initiated postnatally in those fetuses which were delivered by caesarean section while supported by ECMO. It is therefore unlikely that the use of extracorporeal circulation by itself could have influenced our results. It is difficullt and perhaps naive to try to isolate a single factor responsible for the establishment of continuous breathing at birth because physiologically many changes happen simultaneously. Howewer, the use of an ECMO system provides control of fetal blood gases before, during and after cond occlusions.

The exclusion of the umbilical circulation after cord occlusion has been proposed as a crucial mechanism for the initiation of breathing at birth. Both Blanco et al. (1987b) and Adamson et al. (1987) performed. experiments in which the lungs were mechanically expanded with air or $\mathrm{O}_{2}$ in utero to support the fetus after cord occlusion. They reported that breathing activity was established within 138 seconds (Blanco et al. 1987b) and $6 \pm 1 \mathrm{~min}$ (Adamson et al. 1987), being associated with arousal (nuchal muscle activity during $\mathrm{LV} \mathrm{ECOG}$ ), although fetal $\mathrm{PaCO}_{2}$ 
rose to very high levels after occluding the cord. It is interesting that breathing activity stopped within min of cord release despite the fact that fetal $\mathrm{PaCO}_{2}$ was still at very high levels, suggesting involvement of a humoral factor dependent on the placental circulation. Our data showed that cord occlusion, in the absence of a substantial rise in $\mathrm{PaCO}_{2}$ and in the absence of arousal, did not initiate breathing.

Recently, Adamson, Kuipers \& Olson (1991) attempted to control fetal $\mathrm{PaCO}_{2}$ using a high frequency ventilation system. Under these conditions the mean breathing was stimulated significantly after $20 \mathrm{~min}$ of umbilical cord occlusion compared to a 20 min control period. Adamson et al. (1991) suggested that this was due to a slow disappearance of a respiratory modulator after cord occlusion. Our study was designed to observe the presence of breathing within 5 min of cord clamping and not mechanisms involved in the maintenance of breathing thereafter. There are several modulators which can regulate breathing activity directly as endorphins and adenosine (Chernick 1981, Grunstein et al. 1981, Koos et al. 1990b, McQueen 1983, Santiago \& Edelman 1985), prostaglandins or prostaglandin synthetase inhibitors, (Kitterman et al. 1979, Kitterman et al. 1983, Murai et al. 1987, Wallen et al. 1986) or indirectly through peripheral chemoreceptor discharge (Bennet \& Hanson 1990a). Our experiments did not however support the hypothesis that abrupt changes of such modulators in fetal plasma after exclusion of the placenta were responsible for the onset of breathing at birth.

Initiation of breathing after cord occlusion was no more frequent in the fetuses in group II in which $\mathrm{PaCO}_{2}$ was allowed to increase by ca. $34 \%$ than in group IA in which $\mathrm{PaCO}_{2}$ was held at control. In group II $\mathrm{PaO}_{2}$ was held at levels which were hyperoxic for the fetus, although not for the neonate. This might in part offset the effect on oxygen content produced by the addition of maternal blood to the fetal circulation ( $\mathrm{P}_{50}$ adult blood higher than fetal blood). Nevertheless fetal breathing movements occurred on only one of eleven accasions. In group IB $\mathrm{PaO}_{2}$ increased to the same level as in group II but breathing activity was observed in 5 out of 8 and 1 out of 11 experiments respectively, and fetal breathing never became continuous. These experiments support earlier studies of Blanco et al. (1991) in which raising the fetal $\mathrm{PaO}_{2}$ to neonatal levels with ECMO failed to induce changes in the incidence of breathing; nor did such breathing occur when fetal $\mathrm{PaO}_{2}$ was raised by expanding the lungs with mechanical ventilation (Blanco et al. 1987 a).

The recent study of Baier, Hasan, Cates, Hooper, Nowaczyk \& Rigatto (1990) also provided evidence that cord occlusion by itself did not induce continuous breathing. They observed continuous fetal breathing movements in chronically instrumented fetal sheep when the lungs were expanded with hyperoxic gas to elevate $\mathrm{PaO}_{2}$ to about $200 \mathrm{mmHg}$, but not if the $\mathrm{PaO}_{2}$ was elevated to only about $100 \mathrm{mmHg}$. If the fetuses were breathing, subsequent cord occlusion increased the intensity of this breathing; however, if they were not breathing, cord occlusion induced breathing, but this was associated with a rise in $\mathrm{PaO}_{2}$ to about 190 mmHg. However, the unphysiologically high levels of $\mathrm{PaO}_{2}$ used in most of these experiments raise additional questions, as such levels of 
$\mathrm{PaO}_{2}$ produce cerebral vasoconstriction (Blanco et al. 1988): a rise in the $\mathrm{PaCO}_{2}$ in the environment of the medullary chemoreceptors might therefore be involved in the stimulation of breathing. However, it is clear that an elevation of $\mathrm{PaCO}_{2}$ alone is not sufficient to produce continuous breathing: Baier et al. (1990) did not induce continuous breathing after clamping the cord in 5 fetuses in which $\mathrm{PaO}_{2}$ was not ellevated but $\mathrm{PaCO}_{2}$ rose to about $90 \mathrm{mmHg}$, and in our experiments in which $\mathrm{PaCO}_{2}$ was elevated substantially (Group IB) breathing only occurred in 5 out of 8 experiments and then only during LV ECoG.

Recently a relationship between initiation of breathing and gestational age has been reported (Hasan \& Rigaux 1991). These authors could not induce fetal breathing movements in fetal lambs below 134 days of gestation by increasing fetal $\mathrm{PaO}_{2}$ to neonatal levels. It is well known that premature infants initiate breathing as early as 22 weeks gestational age. Therefore we do not think that gestational age was a factor which impaired the initiation of breathing in our work. Furthermore, fetal $\mathrm{PaO}_{2}$ is not expected to be above fetal levels, actually lower, with the initiation of breathing at birth. Hyperoxia can not play a role in the initiation of breathing at birth.

The role of hypercapnia at birth has already been suggested in a previous report (Blanco et al. 1987b) in which the time to the onset of breathing was measured under different conditions in terms of fetal blood gases. It is well established that hypercapnia produces an increase in incidence, amplitude and frequency of fetal breathing movements (Boddy et al. 1974, Bowes et al. 1981b, Chapman et al. 1980, Dawes et al. 1983) and this response is present by 0.5 of gestation in fetal lambs (Ioffe et al. 1987). It is not known whether the response to hypercapnia involves the peripheral or the central chemoreceptors, although involvement of the latter seems more likely as the response occurs in hyperoxia, which reduces the $\mathrm{CO}_{2}$ sensitivity of the peripheral chemoreceptors (Lahiri et al. 1978). Our results (group IB) supported the idea that hypercapnia played a role in the initiation of breathing after cord occlusion. However it is clearly not the only factor involved since breathing did not become continuous but occurred only in LV ECoG.

Fetal temperature remained constant during 19 cord occlusions, in 3 experiments temperature decreased, in 2 experiments temperature increased. This is at variance with reports showing an increase in fetal temperature of $0.9^{\circ} \mathrm{C}$ after cord occlusion in oxygenated $\left(\mathrm{PaO}_{2} 12.6 \mathrm{kPa}\right)$ fetal lambs (Power et al. 1986). In our experiments we did not observe these changes. This difference in obserwations could be explained by the differences in fetal oxygenation (Dawes \& Mott 1964, Power \& Longo 1975 ) and by the fagt that the fetuses in our study were still circulated by the ECMO system.

In summary, our data showed that breathing activity was only present in only 11 of 12 occasions within 5 min after cord occlusion when fetal $\mathrm{PaCO}_{2}$ was held constant. However breathing was more often (but not always) present when fetal $\mathrm{PaCO}_{2}$ increased. This finding further emphasizes that multiple mechanisms are involved in the initiation of breathing at birth. Our results do not allow us to support the hypothesis 
that the exclusion of the umbilical circulation is responsible for the initicution of breathing at birth. The disappearance of such putative placental factor(s) may of course facilitate the maintenance of breathing once it has started after birth. 


\section{Chapter 8}

\section{Initiation and maintenance of continuous breathing at birth}

II.M. Kuipers, W.J. Maertzdorf, D.S. de Jong "M.A. Hanson" and C.E. Blanco.

Dept. of Neonatology, Dept. of Cardiothoracic Surgery \& Dept. of Extra Corporeal Circulation', Academic Hospital Maastricht, University of Limburg, Maastricht, the Netherlands, Dept. of Obstetrics \& Gymaecology22, University College London, London, United Kingdom.

Submitted 


\section{Abstract}

Changes in $\mathrm{PaCO}_{2}$ and temperature, normally occurring at the moment of birth, may play a role in the initiation and maintenance of continuous breathing. To investigate this mechanism we instrumented 5 fetal lambs of 133-135 days of gestation for chronic reconding a cord occluder and catheters for later connection to an extracorporeal membrane system. ECMO was initiated in utero at a flow rate to stpport the fetus after cord occlusion, then the fetuses were delivered into a warm saline bath. Breathing activity was periodically present before connection to the ECMO system and on ECMO during normocapnia/normoxia but near delivery there were no breathing movements present since all ewes were in labour. After delivering the fetuses in a warm saline bath breathing movements were periodically present. After 36-192 min breathing activity became continuously present in all animals $(\mathrm{pH} 7.20 \pm 0.04$, $\mathrm{PaCO}_{2} 7.35 \pm 0.16 \mathrm{kPa}$ and $\mathrm{PaO}_{2} 12.78 \pm 2.51 \mathrm{kPa}$, core temperature had fallen by a mean of $1.2^{\circ} \mathrm{C}$ ). Furthermore, breathing activity stopped by decreasing $\mathrm{PaCO}_{2}$. Maintenance of fetal $\mathrm{PaCO}_{2}$ and temperature after cord occlusion delays the establishment of continuous breathing. The level of $\mathrm{PaCO}_{2}$ is important in the maintenance of breathing activity during the first few hours of life.

\section{Introduction}

Fetal breathing movements are present from early in gestation. After differentiation of electrocortical activity into LV ECOG and HV ECoG, fetal breathing movements are only present during LV ECoG. The transition from episodic fetal breathing to continuous neonatal breathing occurs at a time when many other changes occur, such as exclusion of the placenta, lung expansion with air, $\mathrm{O}_{2}$ and $\mathrm{CO}_{2}$ contact with the airway, decrease in pulmonary wascular resistance and an increase in pulmonary blood flow etc. Furthermore, the newborn is exposed to new afferent input like light, sound, touch and changes in temperature. Not all of these. changes have the same importance. It is difficult to isolate any single factor as being responsible for the initiation of continuous breathing activity at birth.

It is reported previously that hypercapnias stimulates fetal breathing. activity and an increase in $\mathrm{PaCO}_{2}$ is involved in the initiation of continuous breathing at birth (Blanco et al. 1987b). Fast peripheral cooling resulted in continuous breathing activity in utero and after birth (Harned \& Ferreiro 1973, Gluckman et al. 1983). Therefore, at decrease in peripheral temperature might be involved in the initiation of continuous breathing activity at birth. Recently, it has been speculated that after cord occlusion at birth, breathing activity can be initiated due to the disappearance of respiratory inhibitors produced by the placenta (Adamson et al. 1987, Blanco et al. 1987b). However, breathing activity 
was not initiated within 5 min of cord occlusion in chronicle instrumented fetal lambs when the fetus was normocapnic (Kuipers et al. 1992).

The relative importance and interaction of the mechanisms involved in the initiation and maintenance of continuous breathing at birth are not well understood. In order to clarify further the mechanism for the initiation and further maintenance of breathing at birth we have used an ECMO system in chronically instrumented fetall lambs. The aim of this work was to study the role of $\mathrm{PaCO}_{2}$ and temperature on the initiation and maintenance of continuous breathing after birth.

\section{Materials and methods}

Experiments were performed on unanesthetized chronically instrumented fetal sheep in utero (see Chapter 3 ).

Recordings were started within 48 hours after surgery. Seventy-two hours after surgery, the fetuses were connected to the ECMO system. Baselline recordings on ECMO were started at least one hour after connection to the ECMO system. To study the initiation and maintenance of continuous breathing activity after birth the fetuses were delivered while on ECMO into a warm saline bath, using the following procedure. The pump flow was increased to approximately $350-500 \mathrm{~mL} / \mathrm{min}$, then the umbilical cord was occluded. The ewe was sacrificed by injecting $20 \mathrm{ml}$ Euthesate iv. Since the umbilical circulation was interrupted the fetus was not affected. The membrane lung was supplied with a gas composed of $1.5-1.9 \mathrm{l} / \mathrm{min} \mathrm{O}_{2}$, and $0.1 \mathrm{l} / \mathrm{min} \mathrm{CO}_{2}$ at a flow rate of $2.0 \mathrm{l} / \mathrm{min}$. Adequate $\mathrm{O}_{2}$ delivery to the fetus was achieved in two ways. First, the $\mathrm{O}_{2}$-flow to the membrane lung was increased to achieve a higher post-membrane saturation, secondly the pump flow was increased (to $350-500 \mathrm{ml} / \mathrm{min}$ ). The abdomen of the ewe was opened, the uterus was partly visualized and opened very carefully at the place were the fetal catheters, tubing and electrodes were exteriorized. A glove filled with warm saline was placed over the fetal head until the fetal head was under water in the bath, to avoid expanding of the fetal lung with air. The fetus was exteriorized down to the umbilical cord and the cord was cut. The fetus was delivered and placed in a warm saline bath $\left(39.5^{\circ} \mathrm{C}\right)$, taking good care of the catheters, tubing and electrodes cables. The temperature of the bath was allowed to decrease slowly, therefore first peripheral and then central temperature decreased.

Recordings were obtained before ECMO, on ECMO during normocapnia/normoxia, before and after delivering the fetuses in a warm saline bath. The temperature of the saline bath was allowed to decrease slowly, resulting in a decrease of central temperature of the lambs. The $\mathrm{PaCO}_{2}$ level was maintained constant. Blood samples were taken every 15 min, neonatal blood gases and $\mathrm{pH}$ were measured. After establishing continuous breathing activity neonatal $\mathrm{PaCO}_{2}$ was decreased by decreasing the concentration of $\mathrm{CO}_{2}$-flow to the membrane lung. Mild hypocapnia was defined as a fetal $\mathrm{PaCO}_{2} 1$ to $2 \mathrm{kPa}$ less than baseline. 
Pressure deflections representing uterine activity were seen on tracheal pressure and blood pressure channels. Labour was defined when uterine contractions occurred with a frequency $\mathrm{p}<0.05$ per $10 \mathrm{~min}$ and pressure deflections of $12-30 \mathrm{mmHg}$.

\section{Results}

Experiments were performed on 5 fetal lambs, at gestational age of 133-135 days.

Baseline recordings were obtained 55-70 hours after the operation, the duration of these recordings being 10 -19 hours. During baseline recordings fetal $\mathrm{pH}$ and blood gases were: $\mathrm{pH} 7.34 \pm 0.02$, $\mathrm{PaCO}_{2} 6.30 \pm 0.22 \mathrm{kPa}$, and $\mathrm{PaO}_{2} 2.88 \pm 0.37 \mathrm{kPa}$.

Nuchal EMG was associated with HV ECoG. Eye movements $(n=4)$ were associated with LV ECoG and fetal breathing movements. LV ECoG activity accurred $47.6 \pm 2.70 \%$ of the time and the duration of the LV ECoG periods was $15.9 \pm 1.87$ min. Fetal breathing activity was present for $31.7 \pm 3.16 \%$ of the time, the length of periods being $8.23 \pm 1.13 \mathrm{~min}$. The incidence of breathing activity during LV ECoG was $65.8 \pm 3.67 \%$.

On the third day post-surgery the fetuses were connected tho the ECMO system. The periods of normocapnia/normoxia were studied to control for possible effects of ECMO on fetal behaviour. Fetal pH and blood gases during these baseline recordings on the $\mathrm{ECMO}$ system were: pH $7.37 \pm 0.01, \mathrm{PaCO}_{2} 6.22 \pm 0.06 \mathrm{kPa}$, and $\mathrm{PaO}_{2} 3.57 \pm 0.36 \mathrm{kPa}$.

Two ewes went into labour. There were no breathing movements present while these 2 fetuses remained in utero on ECMO during these hours of labour. In these 2 fetuses we could not analyze fetall breathing activity during a control period on ECMO. Nuchal EMG activity was associated with HV ECoG activity. Rapid eye movements were associated with LV ECOG activity and fetal breathing movements. The incidence of LV ECOG was $47.3 \pm 3.3 \%$ of the time and the length of the LV ECoG periods was $15.6 \pm 2.2 \mathrm{~min}$. Breathing, activity was present for $36.0 \pm 4.0 \%$ of the time, the length of fetal breathing periods being $9.2 \pm 1.8 \mathrm{~min}$. The incidence of breathing activity during $\mathrm{LV} \mathrm{ECoG}$ was $75.3 \pm 3.2 \%$ 。

Later, just prior to cord occlusion all fetuses were exposed to spontaneous labour , therefore there were no breathing movements present before the cord occlusion. Fetal $\mathrm{pH}$ and blood gases during the recordings on the ECMO system just prior to cord occlusion were: $\mathrm{pH} 7.34 \pm 0.02$, $\mathrm{PaCO}_{2} 7.62 \pm 0.68 \mathrm{kPa}$, and $\mathrm{PaO}_{2} 5.14 \pm 0.65 \mathrm{kPa}$. Nuchal EMG activity remained associated with HV ECoG activity. Rapid eye movements were associated with LV ECoG activity.

After delivering the fetuses into the saline bath all neonates showed periodic breathing activity, these breathing movements being associated only with LV ECoG. Neonatal pH and blood gases during this period were: pH 7.25 $\pm 0.03, \mathrm{PaCO}_{2} 7.08 \pm 0.31 \mathrm{kPa}$ and $\mathrm{PaO}_{2}$ $14.96 \pm 6.44 \mathrm{kPa}$. LV ECoG was present in $53.9 \pm 6.1 \%$ of the time, 

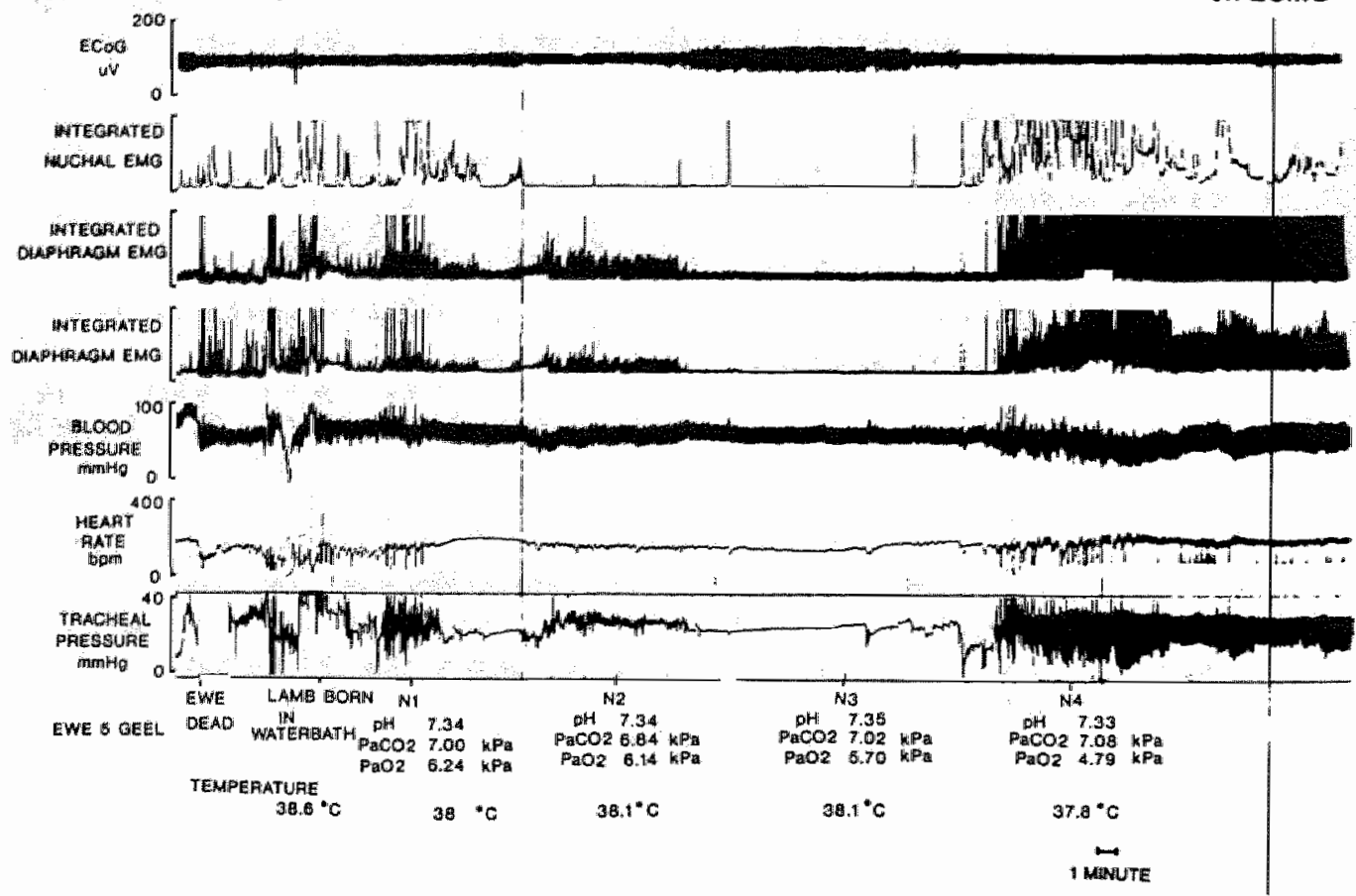

Figure 8.1

Paconding of a newborm lamb at 133 days gestation of approximately 1 hour, 6 days after surgery and connected to the ECMO system. Tracings are from the top: ellectrocortical activity, integrated nuchal EMG, integrated diaphragm EMG, integrated diaphragm $E M G_{1}$ blood pressure, heart rate and tracheal pressure. Bllood gas and $\mathrm{pH}$ samples were taken all the indicated times. Note that breathing movements were still present periodically for 36 min afler delivering the newborn lamb into the saline bath. Breathing activity became present continuously after $36 \mathrm{~min}$. Nuchal muscle activity was still modulated by alectrocontical activity.

breathing movements were present $16.6 \pm 4.0 \%$ of the total time and $30.8 \pm 3.0 \%$ during LV ECoG. Nuchal EMG activity was associated with HV ECoG activity in 4 experiments, in 1 experiment nuchal EMG was also present during LV ECoG (see figure 8.1). Rapid eye movements $(\mathrm{n}=3$, in one experiment electrodes were accidentally cut) were associated with LV ECoG activity and breathing movements.

After 36-192 min breathing activity became continuously present in all animals, breathing movements were continuously present both during LV ECoG and HV ECoG. At this time fetal central temperature had fallen by a mean of $1.2^{\circ} \mathrm{C}$. At the time breathing activity was initiated continuously fetal blood gases and pH were: $\mathrm{pH} 7.20 \pm 0.04, \mathrm{PaCO}_{2}$ $7.35 \pm 0.16 \mathrm{kPa}$ and $\mathrm{PaO}_{2} 12.78 \pm 2.51 \mathrm{kPa}$. Nuchal EMG activity was present during both $\mathrm{LV}$ ECOG and HV ECOG, eye movements remained associated with LV ECoG. 
This continuous breathing activity was very dependent on the level of $\mathrm{PaCO}_{2}$ since in all instances it could be stopped by decreasing $\mathrm{PaCO}_{2}$ $\left(\mathrm{pH} 7.44 \pm 0.02, \mathrm{PaCO}_{2} 3.97 \pm 0.46 \mathrm{kPa}\right.$ and $\mathrm{PaO}_{2} 25.79 \pm 6.78 \mathrm{kPa}$ ).

\section{Discussion}

Our results showed that breathing activity of fetuses delivered into a warm saline bath after cord occlusion and supported on ECMO was still periodic for 36-192 min. We could also show that this activity became continuous when fetal central temperature was decreased by $1.2^{\circ} \mathrm{C}$ by allowing the temperature of the bath to decrease while $\mathrm{PaCO}_{2}$ was maintained. Furthermore, this breathing seemed to be dependent on the level of $\mathrm{PaCO}_{2}$ since it could be stopped or reversed to a periodic pattern by decreasing neonatal $\mathrm{PaCO}_{2}$.

The establishment of continuous breathing at birth has been the subject of several studies. Previous investigators reported the influence of different stimuli on breathing activity at the moment of birth by means of acute experiments (Harned \& Ferreiro 1973, Condorelli \& Scarpelli 1975, Scarpelli et al. 1977, Moss et al. 1983). In those experiments fetal and environmental temperatures, fetal blood gases and $\mathrm{pH}$ were not always controlled and it is known that these factors can influence breathing activity. Therefore, the use of ECMO seemed an appropriate method to study mechanisms involved in the initiation of breathing activity since the fetus could be maintained on ECMO while fetal $\mathrm{PaCO}_{2}$ and skin and core temperature could be controlled when delivered into a saline bath.

The exclusion, after cord exclusion, of respiratory modulators has been proposed as a crucial mechanism for the initiation and maintenance of breathing at birth (Adamson et al. 1987, Blanco et al. 1987b, Adamson 1991, Alvaro et al. 1993). At birth the only change in respiratory control is the presence of breathing during HV ECoG which together with the usually present breathing activity during $\mathrm{LV}$ ECoG results in continuous breathing. Therefore, this fetal respiratory modulator would have had only an effect on the inhibition during $\mathrm{HV}$ ECoG. It will have to be demonstrated that the substance responsible for periodic fetal breathing is the same modulator that disappears after cord occlusion and allows continuous breathing to happen. Moreover, we will have to distinguish or separate the mechanism involved in the initiation and those involved in the maintenance of breathing. Alvaro et al. (1993) infused placental extracts to fetuses presenting continuous breathing obtained by an unphysiologically high $\mathrm{PaO}_{2}$. These experiments showed that the placentat contains some substances which can inhibit breathing activity. However, there are problems $s^{t}$ with the design of the experiment. First, it is not clear whether the breathing obtained is spontaneous breathing activity or just a response to unphysiologically high $\mathrm{PaO}_{2}$ usually associated with arousal. Furthermore, it is possible that the placenta contains or produces substances which can depress or inhibit breathing activity but are not responsible for the inhibition during $\mathrm{HV} \mathrm{ECOG}$.

Our previous (Kuipers et al. 1992) and present work does not support the view that exclusion of respiratory modulators produced by the 
breathing at birth for the following reasons. First, neonatal breathing was not established after cord occlusion whenever $\mathrm{PaCO}_{2}$ was maintained (Kuipers et al. 1992). Secondly continuous breathing was not established until temperature decreased with $\mathrm{PaCO}_{2}$ increased or maintained constant.

Breathing activity before and after birth is dependent on the level of $\mathrm{PaCO}_{2}$ since we observed that in utero continuous breathing could be stopped by decreasing the level of $\mathrm{PaCO}_{2}$ (Kuipers et al. 1994c). It is also now clear that the level of $\mathrm{PaCO}_{2}$ plays an important role in the initiation of breathing activity at birth (Blanco al. 1987b). For example, the initiation of continuous breathing activity is delayed during hypocapnia and stimulated during hypercapnia (Blanco et al. 1987b).

After birth there is a decrease in peripheral and central temperature of the newborn. It is known that peripheral cooling stimulates breathing activity in utero and after birth (Harned \& Ferreiro 1973, Gluckman et al 1983). In our experiments breathing activity remained present periodically for 36-192 min after delivering the fetuses into a saline bath. At the time at which breathing activity became present continuously the temperature of the bath was decreased resulting in a decrease of fetal peripheral and centrall temperature. This indicates that a decrease in fetal temperature is an important factor involved in the initiation of continuous breathing.

So far it is well demonstrated that fetal breathing activity is dependent on the level of $\mathrm{PaCO}_{2}$ but it is not very well known what the role of oxygen availability is. In utero the fetus is exposed to a low $\mathrm{PaO}_{2}$ and since hypoxemia is inhibitory of fetal breathing activity it could be speculated that there is a continuous tonic inhibition only overridden by LV ECoG and this would be the mechanism by which fetal breathing activity is periodic. This is suggested by experiments where breathing activity became continuously present during hyperoxia (Baier et al. 1990, Hasan et al. 1991). However, these experiments were associated with an unphysiological increase of $\mathrm{PaO}_{2}$ which is known to cause a decrease in cerebral blood flow. Furthermore, there is an increase in pulmonary blood flow bringing blood $\mathrm{CO}_{2}$ content in contact with lung receptors sensitive for $\mathrm{CO}_{2}$ (Blanco et al. 1988 , Green \& Sheldon 1983). Furthermore, in the experiments of Baier et al. (1990) and Hasan et al. (1991) there was an increase in $\mathrm{PaCO}_{2}$ and a decrease in $\mathrm{pH}$ known stimulators of breathing activity. In chronically instrumented fetal lambs, raising fetal $\mathrm{PaO}_{2}$ to neonatal levels (8-12 kPa) failed to induce continuous breathing activity (Blanco ef al. 1987a, Blanco et al. 1991). Our results were consistent with the latter. Breathing did not become present continuously despite the increase in $\mathrm{PaO}_{2}(14.96 \pm 6.44 \mathrm{kPa})$ for $36-192$ min. Furthermore, oxygenation cannot have a role in the initiation of continuous breathing since it increases after the initiation of breathing. Thus our experiments do not support the hypotheses that an increase in $\mathrm{PaO}_{2}$ at the time of birth is involved in the initiation of continuous breathing.

It has been suggested that the ability to initiate continuous breathing is dependent on the maturity of the fetus ( $\mathrm{GA}>134$ days) (Hasan et al. 1991, Baier et al. 1992). In our experiments we showed that breathing activity became continuous even in fetuses of 133 days of gestation. This is lately confirmed by Tiktinsky et al. (1994). 
In summary, maintenance of fetal $\mathrm{PaCO}_{3}$ and temperature after cord occlusion delays the establishment of continuous breathing. The level of $\mathrm{PaCO}_{2}$ is important in the maintenance of breathing activity in the first few hours of life. 
Chapter 9

General discussion 
Fetal breathing movements are present in utero from early in gestation. In late gestation breathing movements are present periodically. The transition from periodic fetal breathing to continuous neonatal breathing has to happen within a few minutes, and has to be effective in order to provide oxygenation. The mechanism or mechanisms involved in the control of breathing in utero are not fully understood. The presence of fetal breathing movements provides the opportunity to study the development of the control of respiration when the system is not used for survival. Furthermore, the study of the control of breathing in utero is very important since it can help to explain situations after birth like apnea in premature infants or infants with congenital hypoventilation syndrome, situations that reminds the peculiar control of breathing in utero. Any theory to explain the control of breathing in utero must consider two questions: why are fetall breathing movements present? and why are they absent during HV ECoG?

The aim of the thesis was to study mechanisms inwolved in the controll of fetal breathing activity and in the initiation of continuous breathing at birth. Our first objective was to study the mechanism involved in determining the presence of breathing activity in utero by investigating whether the incidence of fetal breathing movements could be affected by the level of $\mathrm{PaCO}_{2}$. Secondly, we investigated whether the stimulatory response of hypercapnia on fetal breathing is already present in utero and whether increased afferent input produced by cooling might change the sensitivity for $\mathrm{CO}_{2}$ overriding the central inhubition during HV ECoG. This could shed some light on the mechanisms involved in the initiation of continuous breathing at birth. Another interesting phenomenon is the observation that fetal hypoxemia inhibits fetal breathing and behavioral activity. The mechanism responsible for this is still under discussion. We examined the possible role of maternal or placental substances released or triggered during hypoxemia which could act on the ferus. Our final objective was to study further the influence of substances produced by the placenta on the control of breathing activity. Information on the role of the placenta in controlling fetal activity is scarce. Our objective was to study whether exclusion of the placenta, and therefore the disappearance of some theoretically substances produced by it could play a role in the complicated change from periodic to continuous breathing at birtli.

The control of breathing activity in utero and the initiation of continuous breathing at birth has been studied in unanesthetized chronically instrumented fetal lambs in utero over the last 20 years. This was studied in unanesthetized fetal lambs at different gestational ages by changing maternal conditions and consequently fetal conditions such as: changing maternal $\mathrm{PaCO}_{2}$ (fetal hypercapnia or fetal hypocapnia), changing maternal $\mathrm{PaO}_{2}$ (fetal hypoxemia, fetal hyperoxia), changing maternall temperature (fetal hypothermia, fetal hyperthermia), by increasing or decreasing maternal glucose levels (fetal hypoglycemia, fetal hyperglycemia), by reducing uterine blood flow (fetal bypoxemia) etc. Another approach was to change fetal conditions directly by infusing drugs, ventilating the fetal lung with different mechanical ventilator techniques such as intermittent positive pressure ventilation (IPPV), 
continuous positive airway pressure (CPAP) or high frequency oscillation ventilation $(\mathrm{HFO})$ etc. These methods could introduce unknown variables. For example, exposure of the ewe to hypoxemia, hypercapniat or hypocapnia, results in a change in uterine blood flow (Oakes et al. 1976, Bocking \& Harding 1986, Faucher et al. 1991) or in the case of mechanical ventilation, the lung distension produces a stimulation of receptors such as stretch receptors, irritant receptors, $J$ - receptors and also cardiowascular changes. Moreover, with these techniques it is difficult to change $\mathrm{PaO}_{2}$ or $\mathrm{PaCO}_{2}$ independently. In order to avoid some of these problems it was attractive to use a technique which approaches the fetus directly and clarify possible mechanisms in the interaction between placenta and fetus. This could be achieved by using an extracorporeal oxygen techmique. ECMO systems are well developed (Comish \& Kopotic 1990) and presently used for patient care. The membrane lung presently available is capable of providing excellent gas exchange. Connection of the fetus in utero to the ECMO system allowed us to change fetal conditions independently of the ewe. For example, we had the ability to change fetal $\mathrm{PaO}_{2}$, fetal $\mathrm{PaCO}_{2}$ and temperature and to support the fetus during cord occlusion. Therefore, this technique allowed us to study the role of direct changes in $\mathrm{O}_{2}, \mathrm{CO}_{2}$ and temperature on the control of fetal breathing activity. Furthermore, we were able to design experiments to study the role of the placenta on the control of fetal breathing activity under normoxic and hypoxemic conditions.

The ECMO technique is of course invasive. It requires a sizeable (10-14 Fr) catheter in the right jugular vein and 8-10 Fr catheter in the right carotid artery. Furthermore, fetal blood is in contact with foreign surfaces such as silastic tubing, the membrane lung and it is exposed to changes in shear rate. It is therefore necessary to control for this using the same criteria as previously to judge physiological behavior, using i.e. ECoG activity, fetal breathing movements, nuchal muscle activity, rapid eye movements and cardiovascular parameters, blood gases and $\mathrm{pH}$ (Dawes et al. 1972, Molteni et al. 1980, Clewlow et al. 1983). It is then possible to perform experiments only on fetuses which showed comparable behavioral states, cardiovascular parameters and blood gases and $\mathrm{pH}$ as described in the literature. Under these conditions ECMO allowed us to control fetal blood gases, $\mathrm{pH}$ and centual temperature. In our experiments there was no statistical difference in the incidence or duration of periods of fetal breathing movements or LV ECoG activity before ECMO and during baseline recording periods on ECMO.

Furthermore, after connection to the ECMO system and maintaining fetal blood gases and $\mathrm{pH}$, there was normal cycling of fetal states where nuchal EMG activity was associated with HV ECOG activity and eye movements with LV ECOG activity. There were no statistical differences in blood pressure and heart rate before and on ECMO. In conclusion, there was no abnormal behavioral activity during the baseline periods on ECMO which could influence the results presented in the thesis (chapters $4,5,6,7$ and 8). 


\section{Are breathing movements in utero dependent on the level of $\mathrm{PaCO}_{2}$ ?}

Whether the presence of fetal breathing activity is dependent on the level of $\mathrm{PaCO}_{2}$ or it is just an expression of a sleep state is not known. But why would fetall breathing activity be dependent on $\mathrm{PaCO}_{2}$ ? What would be the advantage of having a response to $\mathrm{CO}_{2}$ in utero since fetal $\mathrm{PaCO}_{2}$ will not be corrected by the increase in fetal breathing activity? $\mathrm{CO}_{2}$ is the end product of aerobic metabolism and it has to be eliminated in order to maintain normal $\mathrm{pH}$. Therefore it is logic that there are chemoreceptors for sensing its level and controlling the regulatory mechanisms of breathing. When these sensors are ready to react is not known but it might be speculated that early in gestation they become sensitive to $\mathrm{CO}_{2}$ and are able to stimulate the regulatory mechanism resulting in fetal breathing activity. It is known that after birth breathing activity and $\mathrm{PaCO}_{2}$ are closely related, since during hypercapnia there is an increase in breathing activity and during hypocapnia apnea occurs (Cunningham et al. 1986, Canet et al. 1993). These questions are not new since maternal hypocapnia is known to result in a decrease in presence of breathing activity of the human and the sheep fetus during the last week of gestation (Boddy et al. 1974, Connors et al. 1988, Marsál et al. 1979). However, an effect of hypoxemia could not be excluded in those experiments since there was a simultaneous decrease in uterine and umbilical blood flow (Oakes et al. 1976). Furthermore, in the human studies the experiments were limited by time and by lack of information of blood gases (Connors et al. 1988, Marsál et al. 1979). We could maintain fetal oxygenation and decrease fetal $\mathrm{PaCO}_{2}$ for at least 2 hours in fetuses of 130-134 days of gestation by using ECMO. During mild hypocapnia, behavioral activity such as the incidence of LV ECoG, the length of periods of $\mathrm{LV} \mathrm{ECoG}$, the presence of eye movements during LV ECoG and the presence of nuchal EMG during HV ECoG did not change. Therefore, the possibility for the fetus to breathe remained the same, however, the incidence of fetal breathing movements decreased significantly. This shows that fetal breathing activity is not only dependent on fetal behavioral activity.

Eventhough we cannot determine since when this mechanism is present, it is clear form our experiment that the level of $\mathrm{PaCO}_{2}$ is an essential determinant of the breathing activity in utero. Thus, the sensors and regulators involved in the control of breathing postnatally are already functional in utero since 130 days of gestation.

\section{Does fetal breathing activity respond to increased levels of $\mathrm{CO}_{2}$ ?}

We already discussed the importance of $\mathrm{PaCO}_{2}$ for the presence of fetal breathing activity. We further studied whether fetal breathing activity can be stimulated by an increased level of $\mathrm{PaCO}_{2}$. It is reported that hypercapnia can stimulate breathing activity in utero (Boddy et al. 1974). 
This is expressed by an increase in frequency, amplitude and incidence during LV ECoG. In those experiments fetal hypercapnia was obtained by inducing maternal hypercapnia, therefore introducing confounding variables such as changes in uterine and umbilical blood flow (Walker et al. 1976) or an increase in plasma epinephrine (Faucher et al. 1991). These results can be real since the fetus is sensitive to the level of $\mathrm{CO}_{2}$ but we wanted to rule out some confounding factors. We obtained fetal hypercapnia by increasing the $\mathrm{CO}_{2}$ concentration of the gas flow which supplied the membrane lung and therefore fetal $\mathrm{PaCO}_{2}$ increased significantly without changing maternal conditions. Our experiments confimed previous findings that fetal hypercapnia stimulates fetal breathing activity. The effect of such 'direct' hypercapnia was an increase in amplitude, frequency and incidence of fetal breathing movements during $\mathrm{LV}$ ECoG. Since there was no change in behavioral activity during hypercapnia in our experiments, there was no change in the total incidence and the length of periods of fetal breathing activity. The physiological response to $\mathrm{CO}_{2}$ is already present in utero without any specific function. Since this mechanism is not important in utero it might be speculated that it is already present (the stimulatory effect of $\mathrm{CO}_{2}$ ) in order to play an essential role in the initiation of continuous breathing at birth. Of course there are many other factors involved at the time of birth which contribute to the establishment of continuous breathing which we will discuss later.

\section{Does the association of hypercapnia and cooling play a role in the initiation and maintenance of continuous breathing?}

The mechanisms involved in the inhibition of fetal breathing activity during HV ECoG are not known. It is known that the inhibition during HV ECoG is of cortical origin and the apnea associated with it might be due to a higher threshold for $\mathrm{CO}_{2}$ during $\mathrm{HV}$ ECoG (Dawes et al. 1983, Johnston \& Gluckman 1989). At the time of birth the inhibition during HV ECoG must be lifted in order to breathe continuously. Since HV ECoG state continues to be present after birth its inhibitory influence must be modified. This could probably happen after birth when there is an increase in afferent input to the brain stem, the cortex and an increase in $\mathrm{CO}_{2}$ production. At birth, there are changes in temperature which increase afferent input and simultaneously produce an increase $\mathrm{O}_{2}$ consumption and $\mathrm{CO}_{2}$ production. The association between cooling and an increase in $\mathrm{CO}_{2}$ production at birth could be thought to be mechanisms involved in the initiation of continuous breathing. We could decrease fetal central temperature by decreasing the temperature of the blood in the ECMO circuit while maintaining skin temperature. In most experiments breathing activity became present continuously. In a]l. experiments there was an increase in nuchal muscle activity during LV ECOG and HV ECoG which most probably reflected shivering.

We showed that central cooling associated with hypercapnia overrides the inhibition of breathing activity during $\mathrm{HV}$ ECoG resulting in 
continuous breathing. It might be speculated that breathing activity could be present during HV ECoG because the extra afferent input produced by central cooling modified the inhibitory state during HV ECoG which changed the threshold for $\mathrm{CO}_{2}$. This question is pursued further by measuring the $\mathrm{CO}_{2}$ response during normothermia and hypothemia.

\section{Are the inhibitory effects on fetal breathing during fetal hypoxemia an indirect effect due to release or production of mediators from the maternal side of the placenta or the ewe?}

In contrast to after birth, inhibition of breathing activity is not only seen during HV ECoG but also during hypoxemia. The decrease in oxygen availability is known to inhibit fetal activity (Blanco et al. 1983b. Bocking \& Harding 1986, Boddy et al. 1974, Koos et al. 1987a). The reasons for the fetal hypoxemic inhibition are not clear but there are two mechanisms proposed involving a) chemosensitive inhibitory area in the lateral pons which senses the change in $\mathrm{PO}_{2}$ in the brain stem or b) substances produced by the ewe or the placenta during hypoxemia which directly inhibit the breathing generators. We therefore designed experiments in the attempt to separate those mechanisms. We exposed the ewe to a hypoxic gas mixture while the fetus was maintained normoxic or mildly hyperoxic on ECMO. The well described fetal hypoxemic response (Boddy et al. 1974, Blanco et al. 1983b, Clewlow et al. 1983, Bocking \& Harding 1986, Koos et al. 1987a) was not seen. This could be explained as follows: If there is a chemosensitive area in the pons it was not stimulated in our experiment since $\mathrm{PaO}_{2}$ did not fall to hypoxemic levels. We could not, however, exclude the remote possibility of a substance produced by the fetal side of the placenta and the membranes since these structures are perfused by the fetus. The design of this experiment remains a challenge.

\section{Does the exclusion of the umbilical circulation and therefore placental modulators play a role in the initiation of breathing at birth?}

At birth breathing must become continuous and for that the inhibition during HV ECOG must be overridden. It is suggested that the inhibitory state during HV ECOG could be maintained through the presence of hormones or modulators produced by the placenta. Therefore, in is very attractive to speculate that the disappearance after cord occlusion of these inhibitory agents produced by the placenta are an important factor allowing the initiation of continuous breathing activity after bith. One of the aims of our study was to examine this hypothesis. If exclusion of the umbilical circulation was the key component in the initiation of continuous breathing at birth it should be initiated within 5 min of clamping the cord regardless of the fetal blood gases, fetal ECoG state and whether or not the fetus was already showing breathing activity. In 
utero after cord occlusion breathing activity did not occur within $5 \mathrm{~min}$ when fetal $\mathrm{PaCO}_{2}$ was kept constant or increased slightly. Furthermore, breathing activity remained present periodically for 36-192 min after delivering the fetuses into a saline bath while $\mathrm{PaCO}_{2}$ was held constant. Therefore, our results do not support the hypothesis that the exclusion of the umbilical circulation is responsible for the initiation of breathing at birth.

Our data suggests that not only $\mathrm{PaCO}_{2}$ but changes in behavioral state, produced by an increase in afferent input such as hypothermia can stimulate breathing aetivity in utero and after birth (Harned \& Ferreiro 1973, Gluckman et al 1983). As earlier discussed cooling associated with hypercapnia can produce continuous breathing before cord occlusion.

Moreover, it is clear that $\mathrm{CO}_{2}$ related mechanisms are already present and important after birth. This is clearly shown in our experiments after birth where breathing activity became continuous when temperature decreased to a certain level. Whenever breathing activity became continuous, it was totally dependent on $\mathrm{PaCO}_{2}$ levels since when $\mathrm{PaCO}_{2}$ was reduced breathing activity stopped.

In contrast to oxygen related mechanisms which are fully developed after $24-48$ hours (Blanco et al. 1984) $\mathrm{CO}_{2}$ sensitivity seems to be already reset shortly after birth. The disappearance of placental modulators after cord occlusion did not become very clear as a crucial mechanism in our study since we could modulate the presence of breathing activity by just changing blood gases. It is not ruled out that the disappearance of these substances play a role in the maintenance of breathing activity. Further studies are being carried out to understand the mechanism. 


\section{References}

Abrams RM Gerhardt KJ and Burchtield DJ. Behawional state transition and local cerebral blood flow in fetal sheep. J. Dew. Physioll. 15: 283-288, 1991.

Adamson SL, Patrick JE, and Challs JRG. Effects of nalosone on the breathing. electrocortical, heart rate, glucose and contisol responses to typoxia in the sheep fetus. I. Dev, Physiol. 6: 495-507, 1984.

Adamson SL, Richardson BS, and Homan J. Initiation of pultwonary gas exchange by fetal sheep in utero. J. Appl. Physiol. 62; 989-998, 1987.

Adamson SL, Kuipers M. and Olson DM. Unibilical cord accluriom stimulates breathing independent of blood gases and pH. J. Appl. Physiol. 70: 1796-1809, 1991.

Adamson SL. Respiratory control during the transition at birth. In: Gluckman PD, and Heymann ME Eds. Perinatal and Pediatric Pathophysiology, a clinical perspective. Edward Annold, London 1 614-617, 1993.

Akagi K, Berdusco ETM, and Challis JRG. Cortisol inhibits ACTH but not the AWP response to hypoxaemia in fetal lambs at days $123-128$ of gestarion. J. Dev. Physiol. 14: 319-324, 1990a.

Akagi $\mathrm{K}$, and Challs JRG. Hormonal and biophysical responses 10 acute hypoxemia in fetal sheep at 07-0.8 gestation. Can. J. Physiol. Pharmacol. Vot. 68: 1527-1532, 1990 b.

Albrecht ED, Crensthaw MC, and Pepe GI. The effect of estrogen on placemial dotwery after fetectomy in baboons. Am. J. Obstet. Gyriecol. 160: 237.241. 1989.

Albrecht $\mathrm{ED}$, and Pepe GI. The placenta remans funchional following fetectomy in baboons. Endocrinalogy 116: 843-845, 1985.

Alcorn D, Adamson TM, Lambert TF, Malioney JE, Ritchie BC, and Robinson PM. Morphological effects of chronic tracheal igaton wh drainage in the ferat hawb. J. Anat. 123: 649-660, 1977.

Alcom D. Adamsom TM. Maloney JE, and Robinson PM. Morphological effects of chronic bilateral phithenectomy or vagotowy th the fetal tamb lung. J. Anat. 130. 683-695. 1980 .

Alexander $G$, and Williams $D$. Shivering and mon-shivering thermogenesis during summit metabolism in young lambs. J. Physioll. 198: 251-276, 1968. 
Alwarez JE, Baier RI, Faijardo CA, Nowaczyk BI, Cates DB, and Rigatto H. The effect of 10 or $O_{2}$, wh the continuous breathing induced by $O_{2}$ ar $O_{2}$ plus cord ocalusion in the fetal sheep. J. Dev. Physiol. 17: 227-232, 1992.

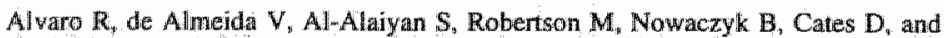
Rigatto H. A placental extract bhibits breahing induced by umbilical cord occlusion th feral sheep. 1. Dev. Physiol 19:23,28, 1993.

Andrews DC, Symonds ME, and Johnson P. Thermoregulation and the cantrol of breathing during non-REM sleep in the developing Lamb. I. Dev. Physiol. 16: 27m, 1991.

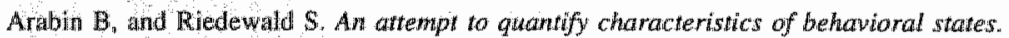
Am J. Perinatol. $9: 115-119,1992$

Bahoric $A$, and Chernick $V$. Electrical actiwty of phrenic nerve and diaphragm in utera. J. Appl. Physiol, 39:513-518, 1975 .

Baier RI, Hatsan SU, Cates DB, Hooper D, Nowaczyk B, and Rigatto H. Effects of varions concentrations of $\mathrm{O}_{2}$ and unibilical cord occlusion on feial breathing and behavior. J. Appl. Plysiol, 68: 1597-1604, 1990.

Baier RJ, Hasan SU, Cates DB, Hooper D, Nowaczyk B, and Rigatto H. Hyperoxemia profoundly alters breathing activity and arouses the fetal sheep. I. Dev. Physiol. 18: $143-150,1992 b$.

Baier RJ, Fajardo C. Alvarez J, Cates DB, Nowaczyk B, and Rigatto H. The effects of gestarional age and labour on the breathing and behaviour response to oxygen and unbilical cord occlusion in the fetal sheep. J. Dew. Physiol. 18: 93-98, 1992a.

Bamford OS, Dawes GS, Hanson MA, and Ward RA. The effects of doxapram on breathing, heart rate and blood prewsure in fetal lambs. Respir. Physiol. 66. 387-396, 1986.

Bantord OS, and Dawes GS. Hypoxia and electrocortical activity in the fetal lamb: effects of brainstem transection and chemoreceptor deneruation. J. Dev. Physiol. 13: $271-276,1990$.

Banford OS, Rivera $\mathrm{A}_{3}$ Tadulan $\mathrm{T}$, and Ellis W. Effects of in utero phrentc nerve secrion on the development of callagen and elastim in lamb lurgs. Am. Rev. Respir. Dis. 146: $1202-1205,1992$

Banzett RB, Coleridge $\mathrm{HM}_{3}$ and Coleridge JCG. I Pulnonary-CO, ventilatory reflex in dogs: effective range of $\mathrm{CO}_{2}$ and resulin of vagal cooling. Respir. Physiol. $34: 121-134$. 1978.

Barcrofi J. Researches on prenatal life. Blackwell Schentific Publications, Oxford 1946.

Bel van F, Roman C. Iwanoto HS, and Rudolph AM. Sympatoadrenal metabolic, and regronal blood flow responses to cold in fenol sheep. Pediatr. Res. 34: 47-50, 1993.

Bennet $\mathbb{L}_{\text {, }}$ Guckman PD, and Johuston BM. The central effects of thyrotrophin-releasing homone on breathing movements and electrocortical activity of fetal sheep. Pediatr. Res. 23: $72 \times 75,1988$.

Bennet L. and Hanson M. The effect of PGE2 on carotid chentoreceptor discharge in

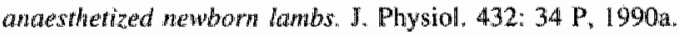


Bennet $\mathrm{L}_{\mathrm{*}}$ Johnston BM, Vale WW, and Giuckman PD. The effects of corticotroptitinreleasing factor and two antagonists on breathing mwentens in fethl sheep. I. Puysiol. 421: 1-11, 1990b.

Berger AJ, Mitchell RA, and Severinghaus JW. Regutarion of raspiration. N. Engl. J. Med. 297: 194-201, 1977.

Berger PJ. Horne RSC, Soust M, Walker AM, and Maloney Ne. Brealihis at birh and the associated blood gas and $\mathrm{pH}$ in the lamb. Respir. Physiol 82: $251+266,1990$.

Berkenbosch A, van Beek JHGM, Olievier CN, de Goede I and Ouanjer PhH, Catral nespiratory $\mathrm{CO}_{2}$ sensitivity at extreme hypocapnia. Respir. Physiof. 55: 95 102, $192 \mathrm{~g}$.

Bissonnette IM, and Hohimer AR. Acute anemic hypoxemic produces a traxwsient depression in fetal respiratary activity. J. Appl. Physiol. 63: 1942-1946, 1987.

Bissonnette JM, Hohimer AR, Chao CR, Knopp SJ, and Notoroberto NF. Theophiyline stimulates fetal breathing movements during hypoxia. Pediatr. Res. 28:83-36, 1990.

Bissonnette JM, Hohimer $\mathrm{AR}$ and Knopp $\mathrm{SI}$. The effect of cemtrally administered adenosine on fetal breathing moventents. Resp. Physiol. 84: 273-285, 1991.

Blanco CE, Dawes GS, and Walker DW. Effects of hypoxia on polysyntiptic hind-find reflexes in stew-born lambs before and after carotid denemation. I. Physiol. 339: $467-474$, $1983 a$.

Blanco CE, Dawes GS, and Walker DW. Effect of hypoxia ons polysynaptic hind-4imb reftexes of unanaesthetized fetal and newhorn lambs. J. Plysiol. $339: 453-466,693$ b.

Blanco CE. Dawes GS, Hanson MA, and McCooke HB. The response to hypoxia of arierial chemoreceptors in fetal sheep and new-born lambs. A. Physiol. 35::25-37, 1984.

Blanco CE, Martin CB Jr., Hanson MA, and McCooke HB. Breathing acthify in fetal sheep during mechanical wentilation of the lungs in utero. Eur. 1. Obstet. Oynecol. Reprod. Biol. 26: 183-192, 1987a.

Blanco CE, Martin CB, Jr., Hanson MA, and McCooke HB. Determinoms of the oniset of continuass air breathing at birht. Eur. J. Obstet. Gynecal. Reprod. Biol. 26: 175-182. 1987b:

Blanco $C E$, Martin CB, Rankin J, Landauer $\mathrm{M}_{\text {and }}$ ahernetton $T$. Changes in fretal organ flow during intrauserine mechanical wentilation with or without oxygen. J. Dew. Phyyiniot. 10: $53+62,1988$.

Blanco CE, Chen V. Maertzdorf W, Bamford OS, and Hanson M. Effect of hypervxia $\left(\mathrm{PaO}_{2}, 50-90 \mathrm{~mm}\right.$ H $\left.\mathrm{g}\right)$ an fetal breathing movements in the ununaesthetized fetat sheep. J. Dev. Physiol. 14: 235-241, 1991.

w.

Bocking $A_{*}$ Adamsón $L$, Cousin $A$, Campbell $K$, Carmichael $L$, Natale $R$, and Patrick J. Effects of imtravenous injections on human fetal breathing movements and gross fetal body movements at 38 to 40 weeks' gestational age. Am. I Obstet. Gynecoll.

142: 606-611, 1982 .

Backing AD, Gagnon R, Milne KM, and White SE Betawiorat activity during protonged hypoxemia in fetal sheep. I. Appl. Physiol. 65: 2420-2426. 1988. 
Bocking $\mathrm{AD}$, and Harding $\mathrm{R}$. Effects of reduced wethe blood fon on electrocontical actiwiny, breathing, and skeletal muscle activity in fetal sheep. Am. J. Obstet. Gynecol. $154: 655-662,1986$.

Boddy $K$, Dawes GS, Fisher $R$, Pinter $S$, and Robunson IS. Foetal respiratory movements" electrocortical and cardiovascular responses to hypoxaemia and hypercapnia in sheep. J. Physiol. $243: 599-618,1974$.

Boddy $K$, Dawes OS, Fisher $\mathrm{R}_{*}$ Pinter $\mathrm{S}$, and Robinson J $\mathrm{S}$ The effects of pentobarbitone and pethidine on foetal breathing movements in sheep. Br. J. Pharmac: 57: $311-317$, 1976.

Bowes $G$, Adamson TM, Ritchie BC, Dowling M, Wilkinson MH, and Maloney JE. Development pattems of respiratory activity in tunanesthetized fetal sheep in ufero. J. Appl: Physioll: 50: 693-700, 1981a.

Bowes $G$, Wilkinson MH, Dowing $M$, Ritchie $B C$, Brodecky $\mathrm{V}$, and Maloney JE. Hypercapnic stimulation of respiratory activity in unanesthetized fetal sheep in utero. J. Appl. Physiol. 50: 701 -708 , $1981 \mathrm{~b}$.

Brown ER, Lawson EE, Jansen A, Chernick V, and Taeusch HW. Regular fetal breathing induced by pilocarpine infusion in the near-tem fetal lamb. I. A.ppl. Physiol. 50: $1348-1352,1981$.

Bryan $\mathrm{AC}, \mathrm{Bow}$ es $\mathrm{G}$, and Maloney $\mathrm{JE}$. Control of breathing in the ferus and the newborn. In: Fishman AP, Cherniack NS, Widdicombe JG Eds. Handbook of Physiology section 3: The Respiratory System, Volume I, part 2. American Physiological Society, Bethesda, U.S.A: 1986, p 621-647.

Canet $\mathbb{E}_{1}$ Praud J-P. Laberge J-M, Blanchard PW, and Bureau MA. Apnea threshold and breathing rhythmichy in newborn lambs. I. Appl. Physiol. 74: 3013-3019, 1993.

Chapman RLK "Dawes GS, Rurak DW, and Wids PL. Breathing movennents in fetal lambs and the effect of hypercapnia. J. Pliysial. 302, 19-29, 1980.

Chernick V. Endorphins and ventilatory control New Engl. J. Med. 304: 1227-1228, 1981.

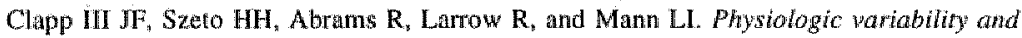
feral electrocortical actiniry. Am. J. Obstet. Gynecol. 136: 1045-1050, 1980.

Clewlow I: Dawes GS, Johnston BM, and Walker DW. Changes in breathing, electroconfical and muscle activity in unawaesthetized fetal lambs with age. I. Physiol. $341: 463-476,1983$

Cohen WR, Piasecki GI, and Jackson BT. Plaswa catecholamines during hypoxemia in! feral lamb. Am. J. Physiol. 243: R520-R525, 1982.

Cohn HE, Sacks EX, Heymanm MA, and Rudolph AM. Cardiowancular responses to hypodemia and acidemia in feral lambs. An. I. Obstet. Gynecoll. 120:817-824, 1974.

Coles SK, Kumar P, and Noble R. Pomine sites inhibiting breathing in anaesthetrzed neonatal lambs. If. Physiol. 409:66P, 1989.

Condorelli S, and Scarpelli EM. Somatic-respiratory reflex and the onset of regular breothing movements in the fetal lambs in utera. Pediat. Res. 9: 879-884, 1975. 


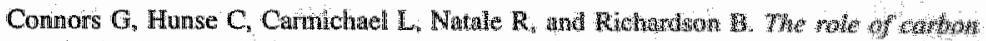

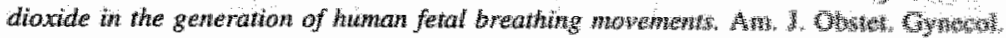
158: $322-327,1988$.

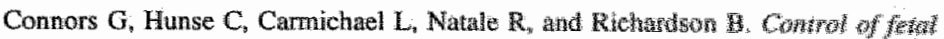

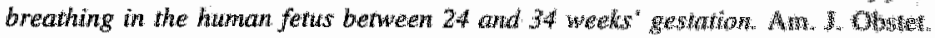
Gynecol. 160: 932-938, 1989.

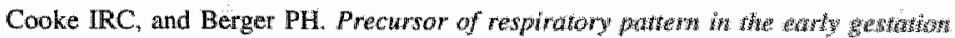
mammalian fetus. Brain Res. 522: 333-336, 1990.

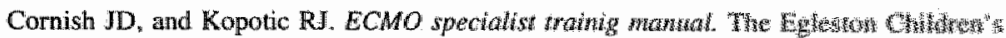
Flospital ECMO-centre, Atlanta Georgia USA, 1990.

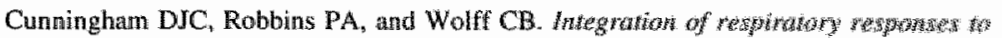

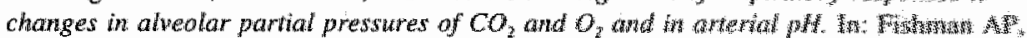

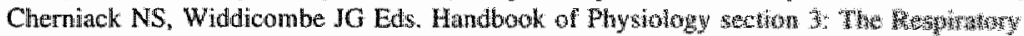

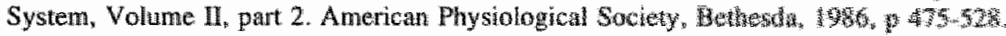

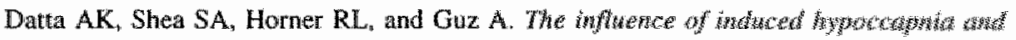

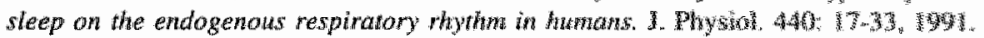

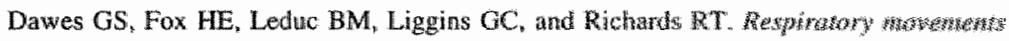

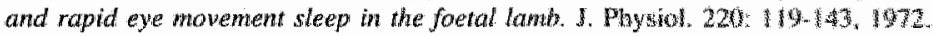

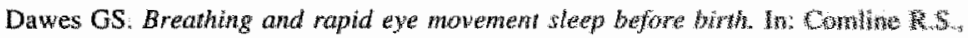
K.W. Cross, P.W. Nathanielsz Eds. Foetal and Neonatal Prysiology, Fyeedinges of the

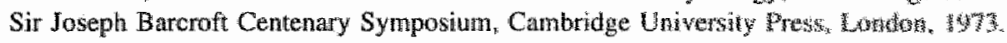

Dawes GS, Gardener WN, Johnston BM, and Walker DW. Actwity of stremcostat masteters

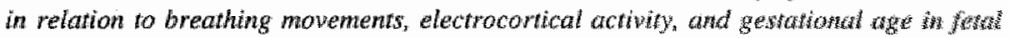
lambs. J. Physiol. 307: 47-48P, 1980.

Dawes GS, Gardner WN, Johnston BM, and Walker DW, Effects of hypercalsnias ant tracheal pressure, diaphragm and intercostal electromyogranis in unamaentitentzed feat lambs. J. Physiol. 326: 461-474, 1982.

Dawes GS, Gardner WN, Johnston BM, and Walker DW. Breathing in fetal lawbs: the effect of brain stem section. J. Physiol. 335: 535-553, 1983

Dejours P. Chemoreftexes in breathing. Physiol. Rev. 42: 335.358, 1962.

Dickson KA, and Harding R. Fetal breathing and pressures in the rrochea and anwhint sac during oligahydramnios in sheep. I. Appt. Physiol. 70: 293-299, 1991.

Eldridge $\mathbb{F L}$, and Millhorn DE. Oscillation, gating, and mewory in the retpiratory control system. In: Fishman AP, Cherniack NS, Widdicombe JG Eds. Handbook of Physiology section 3 The Respiratory System, Volume II, part $H$. American Physiological Society, Bethesda. U.S.A., 1986, p 69-91.

Faber Jj, Anderson DF, Morton MJ, Parkes CM, Pinson CW, Thronburg KL, and Willis DM. Birth, its physiology, and the problems it creates. In: C.T. Johes and $\mathrm{P}$.

Nathanielsz Eds. The Physiological Development of the Fetus and Newbon. Acrulemic press, London, U.K. $1985.371-380$ 
Faucher Dy Laptook AR, Porter $\mathbb{I C}_{\mathrm{r}}$ and Rosenfeld $\mathrm{CR}$. Effects of acuite hypercapnia on materinal and fetal wasopresun and catecholawiune release. Pediatir. Res. $30: 368-374$, 1991.

Fend V. Handboak of Physiology. Section $3:$ the Respiratory Systew Voluthe I. Control of Breathing, part I. Bethesda, American Physiological Society, 1986, 1 15-140:

Ferreira $\mathrm{SH}_{2}$ and Vane JR Prostoglandins: Their discupearince from and release into the circulation. Nature $216: 868-873,1967$.

Fewell IE, LeE CC, and Kitterman JA. Effects of phrenic nerwe section on the respiratory iystem of yetal lambs. I. Appl. Physioll. 51: 293-297, 1981.

Fisk NM, Parkes MI, Moore PJ, Haidar A., Wigglesworth J, and Hanson MA. Fetal breathing dorigg chronic hing liquid loss leading to pubmonary hypoplasia. Early Hum. Dev. $27: 5363,1991$.

Fleming $\mathrm{PJ}$. Bryan $\mathrm{AC}_{\mathrm{x}}$ and $\mathrm{Bryan} \mathrm{MH}$. Finctional immaturiy of pulmonary irritant receprors and apnea in newbown preterm infants. Pediatr. 61: 515-518, 1978.

Fletcher DJ, Hanson MA, Moore PJ, and MJ Parkes, Stimulation of the sheep fetws in wero by sound i. Plyysiol 409: 42 P, 1989.

Gluckman PD, Gunn TR, and Johnstion BM. The effect of cooling on breathing and shivering in whanesthetized fetal lambs in utero. J. Physiol. 343: 495-506, 1983.

Gluckman PD, and Johnston BM. Lestons in the upper lateral pons abolish the hypoxic depression of breathing in wnanaesthetized fetal lambs in utero. J. Physiol. 382:373-383, 1987.

Crunstein MM. Hazinsky TA, and Schlueter MA. Respiratory control during hypoxia in newborn rabbits: implied action of endorphins. I. Appl. Physiol. 51: 122-130, 1981.

Gu $W_{\text {and }}$ andes $\mathrm{CT}$. The effect of elevation of maternal plasma catecholamines on the ferus and placenta of the pregnant sheep. J. Dev. Physiol. 8: 173 186, 1986.

Guerra FA, Savich RD, Wallen LD, Lee CH, Clyman RI, Mauray FE, and Ritterman JA. Prostaglandiw te causes hypoweritarion and apnea in newborn lanbs. I. Appl. Physiol. 64: $2160-2166,1988$

Guerra FA, Sawich RD, Clyman RI, and Kitterman JA. Meclofenamate increases ventilation in lambs. J. Dev. Physiol. 11:16, 1989.

Guissani DA. Moore PJ, Bennet L, Spencer JAD, and Hanson MA. Phentolamine increases the incidence of fetal breathing mowements both thomoxia and iw hypoxia in lem fetal sheep. Ji: Hyiol 425:320P, 1992.

Gumn TR, Johnston BM, Iwamoto HS, Fraser $M$, Nicholls $\mathrm{MG}_{*}$ and Gluckman PD.

Haemodynanic and atecholamine responses to hopothermia in the fetal sheep in wero. J. Dev. Physiol. 7: $24:-249,1985$.

Haddad GO, and Mellins RB. The role w atray receptors in the control af respiration in infants: a vewew. J. Pediatr. 91: 281-286, 1977. 


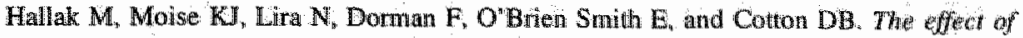

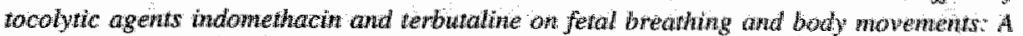
prospective, randomized, dowble bind, placebo-controlled clinical thiol Am. I. Obstet. Gynecol. $167: 1059-1063,1992$.

Hansen NB, Brubakk AM; Bratid D, Oh W, and Stonestreet BS. The effects of wariations in $\mathrm{PaCO}_{3}$ on brain blood flow and cardiac ouph in the newborn phiglet. Pediatr. Res. 18: 1132-1136, 1984.

Hanson MA, Moore PJ, Nijhuis JG, and Parkes MJ. Effects of pilocarpine on breithing noventents in normal chemodenerwated and brain-stem tansectenl fetal sheep. J. Physiol. 400: $415-424,1988$.

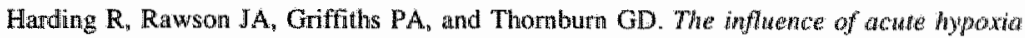
and sleep states on the electrical activity of the cerebelum in the shep feths. Electroencephalogr. Clin. Neurophysiol. 57: 166-173, 1984.

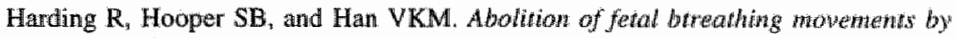
spinal cord transection leads to reductions in fetal lung liquid wolume, fung growh, and IGF-II gene expression. Pediatr. Res. 34: 148-153, 1993.

Harned HS, and Ferreiro J. Initiation of breathing by cold stimulation: Effects of change in anbient temperature on respinatory activity of whe full-term fetal lamb. $\mathrm{j}$. Pediati. 83: $663-669,1973$.

Hasan SU, Bamford OS, Hawkins RL, Gibson DA, Nowaczyk BI, Cates DB, and Rigatto H. The effects of brain-stem section on the breathing and behavioural response to morphine in the fetal sheep. J. Dev. Physiol. 13: 147-155, 1990.

Hasan SU, Lee DS, Githon DA, Nowaczyk BJ, Cates DB, Sitar DS, Pirnsky C, and Rigatto H. Effect of morphine on breathing and behavior in fetal sheep. I. Appl. Physiol. 64: 2058-2065, 1988.

Hasan SU, and Riganx A. The effects of lung distension, oxygenation, and gestational age on fetal behavior and breathing moventents in sheep. Pediatr. Res. 30: 193-201. 1991.

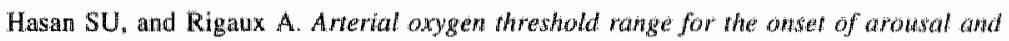
breathing in fetal sheep. Pediatt. Res. 32: 342-349, 1992.

Hedner T, Hedner J, Wesberg $\mathbf{P}$, and Jonason J. Regulation of breanting int whe rat: undicanions for a wole of central adenosine mechanisms. Neuroscience Letters $33: 147-151,1982$.

Himman DJ, and Sizeto HH. Cholinergio influences on slecpwake parterns and breathing movements is the ferus. J. Pharmacol. Exp. Ther, 247: 372-378, 1988 .

Hohimer AR, and Bissonnette JM. Effect of wetabolic acidosis ow feral breahing movememts in wtero. Respir. Physiol. 43: 99-106, 1981.

Hohimer $\mathrm{AR}$, and Bissonnette JM. Vascular Lactic acid infusions do wor alter the incidence of fetal breathing movements or their whibition by acute bypoxemia. Pediatr. Res. 29: 483 486, 1991 .

Hohimer AR, Bissonnette IM, Richardson BS, and Machida CM. Cewwat athental regulation of breathing mowements in fetal lambs. Respir. Physiol. 52; 99.111, 1983. 
Hohimer AR, Richardson BS, Bissonmette JM, and Machida CM. The effect of Hatomethaciw on brewhing mowements and cerebral blood flow and wetabolism in the fetal sheep. I. Dev. Dhysiol. 7: 217-228, 1985.

Woffe $\mathrm{S}$, Jansen $\mathrm{AH}$, and Chernick $\mathrm{V}$. Maturation of spontaneows fetal diaphragmatic activity and fetal response to hypercapnia and hypoxemia. J. Appl. Physiol. 62: 609-622, 1987 .

Iotfe 5, Jansen AH, and Chernick V. Antlysis of respiratory neuronal acivity in fetal Sheep, J. Appl. Physiol. 73: 1972-1981, 1992.

Ioffe $\mathrm{S}$, Jansen $\mathrm{AH}$, Russell $\mathrm{BJ}$, and Cherrick $\mathrm{V}$. Sleep, wakefulness and the monosymaptic reflex in fetal and newborn lambs. Plägers Arch. 388: 149-157, 1980 .

Iwamoto HS, Teitel D, and Rudolph AM. Effects of birth-related events on blood flom distributton. Pediatr. Res. 22: 634-640, 1987.

Jansen $\mathrm{AH}$, de Boeck $\mathrm{C}$, Ioffe $\mathrm{S}$, and Chernick V. Indomethach induced fetal brecthing: mechantssm and site of action. J. Appl. Physiol. 57: 360-365, 1984

Jansen $\mathrm{AH}$, llofe $\mathrm{S}$, and Chernick $\mathrm{V}$. Drug-induced changes in fetal breathing and sleepstate. Can. I. Physiol. Pharmacol. 61: 315-324, 1983.

Jansen AH, Ioffe S, and Chernick W. Sthrulation of fetal breathing activity by betaadrenergic mechanisms. J. Appl. Physiol 60: 1938-1945, 1986.

Jangen AH, Ioffe S. Russell BJ, and Chernick V. Effect of carotid chenoreceptor" denewation on breathing in utero and after birth. J. Appl. Physiol. 51: 630-633, 1981.

Jansen AH, loffe $\mathrm{S}$, Russell BJ, and Chernick $\mathrm{V}$. Influence of sleep state on the response to hypescapnia in fetal lambs. Respir. Physiol. 48: 125-142, 1982.

Johnston BM, and Gluckman PD. GABA-mediated inhibition of breathing th the late gextation sheep fetus. J. Dev. Physiol. 5: 353-360, 1983.

Johnston BM, and Gluckman PD. Lateral pontime leshons effect central chemosiensitivity in whanesthetized fotal lambs. J. Appl. Physiol, 67. 1113-1118, 1989.

Jones SA, Adamson SL, Bishai I. Lees J, Engelberts D, and Cocean F. Eicosanots in third kentricle cerebrospinal fluid of fetal and newborn wheep. Am. J. Physiol. 264: R135-R142, 1993.

Joseph SA, and Walker DW. Carecholamine neurons in fetal brain effects on brecuhing monemenus and electrocorticogram. J. Appl. Physiol. 69: 1903-1911, 1990.

Kanam CM, $\mathrm{O}^{\circ}$ Gredy $\mathbb{I P}$, and Veille JC. Effect of maternal carbondioxide malation on Munan fetal breathing moventents in term and preterm labour. Obstet. Gynecol. 78: 9-13. 1991 .

Kelleman A, Binienda Z, Ding X-Y, Rittenhouse L, Mitchell M, and Nathanielsz PW. Prostaglandin production in the umbilical and uterime circulations in pregnant sheep at 129-136 days gestation. J. Dev. Physiol. 17: 63-67, 1992.

Khazin AF, Hon EH, and Hehre FW. Effects of maternal hyperoxia on the fetus. Am. J. Obstet Gynec 109:628.637, 1971. 
Kitanakia T, Gibert RD, and Longo LD. Maternal responses to long-term hypotemia in Sheep. Am. 3. Physiol. 256: R1340 R 1347, 1989:

Kitterman JA, Liggins $\mathrm{GC}$, Clements JA, and Tooley WH. Srikwlathon of bieathing movements in fetal shep by indibitors of prostaglandin syathesis. J. Dev, Physiol. 1: $453-466,1979$.

Kitterman JA, Liggins $G C$, Fewell JE, and Tooley WH. Inhibition of breathing wovements in fetal sheep by prostaglandins. J. Appl. Physiol: 54: 687-692, 1983.

Kolobow T, Gatinonil L, Tomlinson TA, Pierce JE. Control of breathing using an extracorporeal membrane lumg. Anesthesiology 46: 138-141. 1977.

Koos BJ. Central stimulation of breathing mowements in fetal lambs by prostaglandin synthetase inhibitors. J. Physiol. $362: 455 \times 466,1985$.

Koos BJ, Sameshima $\mathrm{H}_{\mathrm{n}}$ and Power GG. Fetal breathing movenent step wate and cardiovasculas responses to an inhibitor of mitorthondrial ATPase in sheep. J. Dev. Physiol. 8: 67-75, 1986.

Koos BJ, Sameshima H, and Power GG. Fetal breathing, sleep state, and cardionascular responses to graded hypatia in sheep. 1. Appl. Physiol. 62: 1033-1039, 1987a.

Koos BJ, Sameshima H, and Power G. Felal brearhing, sleep statt, and cardiovasculat responses to graded anemia in sheep. J. Appl Physiol. 63: 1463-1468, $1987 \mathrm{~b}$.

Koos BJ, Kitanaka T, Matsuda K, Gilbert RD, and Longo LD Fetal brearhing adaptation to prolonged hypaxaemia in sheep. J. Dev. Physiol. 10: 161-166, 1988a.

Koos BJ, Matsuda $\mathrm{K}$, and Power GG. Fetal breathing and sleep state responses to graded carboxyhemoglobinemia in sheep. J. Appl. Physiol. 65: $2118-2123,19886$.

Koos BI, and Sameshima H. Effects of hypoxaemia and hypercapnia on breathing movements and sleep state in sinoaontic denerwated fetal sheep. J. Dev. Physiol. 10: $131-144,1988 \mathrm{c}$.

Koos BJ, Matsuda K, and Power GG. Fetal breathing and cardiovasculat repponses ta graded methemoglobinemia in sheep. I. Appl. Physiol. 69: 136-140, 1990a.

Koos BJ, and Matsuda K. Fetal breathing, sleep state and cardiovascular mesponses wo aderiosine in shep. J. Appl. Plysiol 68:489-495, 1990b.

Koos BJ, and Doany W. Role of plasma adenosine in breathing responses to hypoxid in feral sheep. I Dev. Physiol. $16: 81-85,1991$.

Koos BJ, Char A, and Daony W. Adenosine stimalates breaning in feral whep with brain srem section. J. Appl. Physiol. 72: 94-99, 1992.

4

Kuipers IM , Maenzdorf WJ, de Jong DS, Hanson MA, Blanco CE. The effect of wild hypocapnia on breathing and behavior in unanesthetized normoxic felal lantbs. I. Appl. Physiol. $76(4)$ : 1476 1480, 1994a.

Kujpers IM, Maertzdorf WJ de Jong DS, Hatison MA, Blanco CE. Initiation and maintenance of continuous breathing at bith. Submilied $1995 \mathrm{~b}$. 
Kupers IM, Mattatorf WJ, de Jong DS; Hangon MA, Blanco CE. The effect of hypercopnia and hypercapina associated with central cooling on behavior in unanesthetized fenal Lambs. Submidided 1995a.

Kuipers 1M, Maertzdorf W, de Jong DS, Hanson MA, Blanco CE. The effect of central

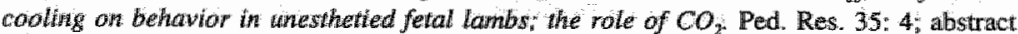
$408,1994 \mathrm{c}$.

Kuipers IM, Maertzdorf WJ, Kewen H, de Jong DS, Hanson MA, Blanco CE. Fietal breaking is not intiated after cord occlusion in the unanesthetized fetal lamb in utero. J. Dew Physiol $17: 233240,1992$.

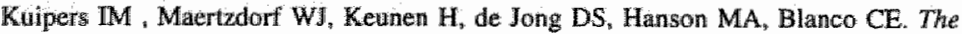
effect of matemal hypoxemia on behawior in unanesthetized normoxic or mildty hyperoxic fetal lambs. J. Appl. Physiol. 76; 2535-2540, 1994.

Kuwimura $\mathrm{T}_{\mathrm{y}}$ Gilbert RD, and Power GG. Effect of cooling and heating on the regional distribution of bload flow in fetal sheep. I. Dev. Physiol. 8: $11-21,1986$.

Lagercrantz $\mathrm{H}$, and Bistoletti $\mathrm{P}$. Catecholamine release in the nawborm infant. Pediatr. Res. 11 : 889-893, 1973.

Lagercrantz $\mathbf{H}_{4}$ Peguignot J, Pequignot J-M and Peyrin L. The first breath of air shimulare noradrenaline narnower in the brain of the newborn rat. Acta Physiol. Scand. 144: $433-438,1992$.

Lahiri $S_{1}$ Mokeshi $A_{1}$ Delaney $\mathrm{RG}_{\text {, }}$ and Fishman AP. Arterial $P_{02}$ and $P_{C O 2}$ stimulus threshold for carotid chemoreceptors and breathing. Respir. Physiol. 34: 359-375, 1978 .

Leduc $B$. The effect of hyperventilation on matewal placental blood flow in pregnant rabbits. J. Physiol. 225: $339-3481972$.

Lee DS, Choy P. Davi M, Caces R, Gibson D, Hasan SU, Cates D, and Rigatto H. Decrease in prostaglandin $\mathrm{E2}$ is not essential for the establishment of continuous breathing at birth in sheep. J. Dev. Physiol. 12: 145-151, 1989.

Levinson G, Shnider SM, deLormier AA, and Steffenson NL. Effects of maternal hyperventilation on uterine blood flow and fetal angenation and acid-base stains. Aruesthesiology $40 ; 340-347,1974$.

Lewis AB, Evans WN, and Sischo W. Plasma catecholamine responses to hypoxemia in feral tambs. Biol. Neonate $41.115-122,1982$.

Liggins GG, Vilos GA, Campos GA, Kitterman JA, and Lee CH. The effect of spinat cond transection on ung development in fetal wheep. J. Dev. Physiol. 3: 267-274, 1981a.

Liggins $\mathrm{GG}_{\text {, Vilos }} \mathrm{GA}$, Campos, GA, Kitterman JA, and Lee CH. The effec of bilareral thoracoplasty on lung dewelopment in fetal sheep. J. Dev. Physiol, 3: 275-282, 1981b.

Malcolm GA and Henderson-5mart DJ. The effect of body tewperature on the ventilatory response to $\mathrm{CO} 2$ in mewatal rats. Twenty first Annual Meeting of the Society for the Study of Fetal Physiology. Paln Cove, Cairns, Australia, July 30-August 3, 1994. Abstract 58 .

Marsál $\mathrm{K}$, Gennser $\mathrm{G}$, Löfgren $\mathrm{O}$. Effects on fetal breathing mowements of maternal challenges. Acta Obstet. Gynecol. Scand. 58: 335-342, 1979. 
Marsland DW, Callahan B, and Shamnon DC. The offerent nagur and regulation of breathing in response to inhaled $\mathrm{CO}_{2}$ in awake newborn lambs. Biol. Neonate 27: 102-107, 1975 .

Martin $\mathrm{CB}$, Ji, Voermans TMG, and Jongsma HW. Effect of reducting wateroplacental blood flow on mowements and on electrocomical activity of fetal sheep. Gynecol. Obstet. Invest. $23: 34,39,1987$.

Matsuda $\mathrm{K}$, Ducsay $\mathrm{C}$ and Koos BJ. Fetal breathing, sleep state ant comliowascular adaptations to anaemia ire shep. J. Physiol. 445: 713-723, 1992.

McCown TJ, Hedner JA, Towle AC, Breese GR, and Mueller RA. Brathtem localization of thyrotropin-rellecasing howdone-induced change in respiration junchow. Brain Res. 373: 189-196, 1986.

McQueen DS. Opoid peptide interactions with respinatory and circulatory systenks. Br. Med. Bull. 39: $77-82,1983$.

Molten RA, Melmed MH, Sheldon RE, Jones MD, and Mesethia G. Induction of fetal

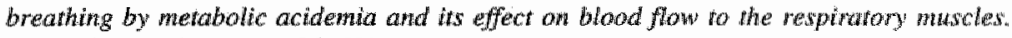
Am J. Obstet. Gyrecol. 136:609-620, 1980.

Moore PJ, and Hanson MA, Control of breathing; central influences. Edited by MA Hanson JAD Spencer, CA Rodeck and D Walters. Fetus and neonate Physiology and clinical applications, Volume 2 Breathing. Press Syndicate Cambridge UK, $109-136$, 1994.

Moore PJ, Parkes MJ, Nijhuis JG, and Hanson MA. The incidence of breathing movements of fetal sheep in mormaxia and hypoxia after peripheral chemodenerwation and brain-stem transection. J. Dev. Physiol, 11: 147-151, 1989.

Moore PJ, Parkes MJ, Noble R, and Hanson MA. Reversible blockade of the secondary fall of wentilation dwring hypoxia in anaesthetized newborn sheep by focal cooling in the brain stem. J. Physiol. 438: $242 \mathbb{P}, 1991$.

Mortola JP, Fisher JT, Smith JB, Fox JS, Weeks $S$, and Willis $D$. Onser of respliration in infants deluvered by cesarean section. I. Appl. Physiol. 52: 716-724, 1982.

Moss IR, and Scatpelli EM. Generation and regulation of breathing an wero: fefal $\mathrm{CO}_{2}$ response test. J. Appl. Physiol. 47: $527-531,1979$

Moss IR, Mautone AJ, and Scarpelli EML Effect of tampenature on regalation of breathing and sleeptwake state in fetal kambs. J. Appl. Physiol. 54:536-543, 1983.

Motoyana $\mathrm{EK}, \mathrm{Rivard} \mathrm{G}_{\text {. Acheson }} \mathrm{F}$ and Cook $\mathrm{CD}$. The effect of changes in matemal pH and $\mathrm{PCO}_{2}$ on the $\mathrm{PO}_{2}$ of fetal lambs: Anessthesiology 28: 891.903, 1967.

Mulder EJH, Boersma M, Meeuse M, Wal van der M, Weerd van de D, Visser GHA. Pattens of breathing molentents in the near-term human ferus: relationshtp to behawiowral states. Early Hum. Dew. 36: 127-135, 1994.

Mura DT, Clymann RI, Matray FE, Lee C-CH, and Kitterman JA. Meclofanamate and prostaglandin $E_{2}$ affect breathing movements independertly of ghcose concentrations in fetal sheep. Am. J. Obstet. Gynecol. 150: 758-764, 1984. 
Murai DT, Lee CH. Wallen LD, amd Kitterman JA. Dewerwation of peripheral chewhreceptors decreases breathing movenuews in fetal sheep. I. Appl Physiol. 59: $575-579,1985$

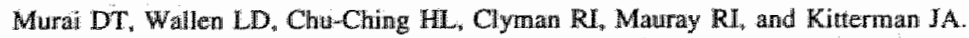
Effects of prostaglatinis on fetal breathing do not inalve pieripheral chemoreceprors. J. Appl. Physiol. 62: $271-277,1987$.

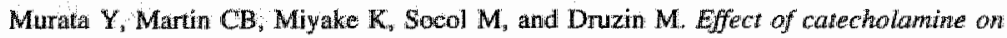
fenal breathing actwity in rhests monkey. Am: J. Obstet. Gynecol: 139: 942-947, 1981.

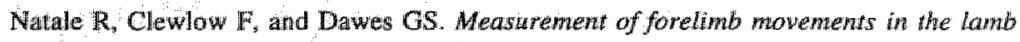
in utero. Am. J. Obstet Gynec. 140:545-551, 1981.

Natale R, Patrick $\mathrm{J}$, and Richardson B. Effects of hwan maternal venous plasma glwcose concentrations on fetal breatiung movements: Am. J. Obstet. Gynecol. 132: 36-41, 1978 .

Nathanielsz PW, Figueroa JP, and Honnebier MBOM. In the thests monkey placental retention after ferectomy at 121 to 130 days" gestation outhasts the normal duration of pregnancy. Am. J. Obstet. Gynecol. 166: 1529-1535, 1992.

Nijhuis $\mathrm{KO}_{\mathrm{b}}$ Precht HFR, Martin CB, jr. and Bots RSGM. Are there behavioral stater in the human ferw.s. Early Hum. Dev. 6: 177-195, 1982.

Noble $\mathrm{R}_{\text {, and }}$ Smith JA. Imhibition of breathing in meonatal kittens by pontine electrical stimulation. Society for the Study of Fetal Physiology. 18 $8^{\text {th }}$ International Meeting. May 7-10, 1991, Abstract 47.

Noble R, and Williams BA. Excitation of neurones in the rostral lateral pons during hypaxia in anaestherized neonatal lambs. J. Physiol. 417:146P, 1989.

$\mathrm{O}^{\prime} \mathrm{Gradly}$ JP, Richardson B, Hohimer AR, and Burry KA. The effect of induced maternat hypencapnia on gross fetal body movements. Am. J. Obstet. Gynecol. 146: 52-56, 1983.

Oakes GK, Walker AM, Ehrenkranz RA, Cefalo RC, and Chez RA. Uteroplacental blacd flow during hyperthemia with and without respimatory alkalosis. I. Appl. Physiol. 41: $197-201,1976$

Okai T, Kozuma $S^{a}$, Shinozuka N, Kuwabara $\mathrm{X}$, and Mizuno M. A stady on the developwent of sleep-makefniness cycle in the muman fetus. Early Hum. Dew 29:391-396, 1992.

Olson DM. Lye SI, and Challis JRG. Prostaglandin concentrations in ovime maternat

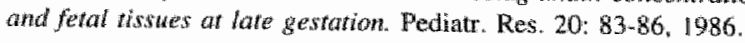

Pappentheimer IR. Fencl $\mathrm{V}$. Heisey $\mathrm{R}$, and Held D. Role of cerebral fluids in conmol of respiration as studied in unanesthetized gaats. Am. J. Fhysiol. 208: 436 450, 1965.

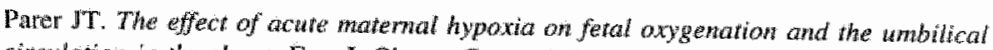
circulation in the sheep. Eur. J. Obstet. Gynecol. Reprod. Biol. $10: 125-136,1980$.

Parkes MI, Moor PI, Moore DR, Fisk NM, and Hanson MA. Behavioral changes in fetal sheep cansed by wibroncoustic stimulation: The effects of cochlear ablation.

Am. J. Obstet Gyrnecol. 164: 1336-1343, 1991. 
Patrick J, Natale R, and Richardison B. Pattems of hwwat fetal breathing acthiny at 34 to 35 weeks" gestational age. An. J. Obstet. Gynecol. 132, 507-513, 1978.

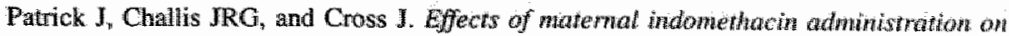
fetal brecthing wowemexts in sheep. J. Dev. Physioll. 9: 295-300, 1987.

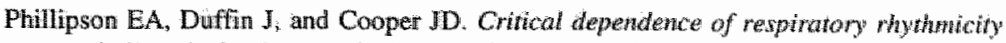
an metabolic $\mathrm{CO}$ load. J. Appl. Physiol. 50:45-54, 1981 .

Phillipson EA, and Bowes G. Control of breathing ditring sleep. In: Fishman AP. Cherniack NS, Widdicombe JG Eds. Handbook of Physiology section 3: The Respiratory System, Volume II, part 2. American Physiological Society, Bethesda, U.S.A, 1986 . p 649-689.

Power GG, Kawantura T. Dale PS, Schroder H, Gilbert RD. Tempenanwe responses following ventilation of the fetal sheep in utero. I. Dev. Physiol. 8: 477-484, 1986.

Power $\mathrm{GG}$, and Longo $\mathrm{LD}$. Placental $\mathrm{O}_{2}$ transfer and fetal constumprion an wayting feral anterial $\mathrm{PO}_{2}$. Fed. Proc. 34: 451. 1975.

Prechtl HFR, Akiyama $Y$, Zinkin $P_{y}$ and Grant DK. Polggraphic studies of the fllt-tom newbon.I. Technical aspects and qualitarive andysis. In. R. Mackeith, Bax Bds. Sudies in Infancy: clinics in developmental medicine, Heinemann Medical Books, London, U.K., $1968,27: 1-21$.

Quilligan EJ, Clewlow F, Johnston BM, and Walker DW, Effect of 5-hydroxytryptophan on electrocorical activity and breathing movements of fetal sheep. Am. J. Obstet. Gynecol. 141: 271-275, 1981.

Reid DL, Jensen A, Phernetton. TM, and Rankin JHG. Relationship between plasma catecholamine levels and electrocortical state in the mature fetal lambs" $\mathbf{J}$. Dew. Physiol. 13: $75-79,1990$.

Richardson B, Hohimer AR, Mueggler $\mathrm{P}$, and Bissonnette J. Effects of glucowe concentration on fetal breathing movements and electrocortical activity in feral lambs. Am. J. Obstet. Gynecol. 142: 678-683, 1982.

Richardson B, Natale R, and Patrick J. Hwman fetal breathing activity during etectwely indaced kabor al rem. Am. J. Obstet. Oynecol. 133:247-255. 1979.

Richardson BS Parick JE, and Abdul-Jabbar A. Cerebrat oxidathe nactabolism in the fetal lamb; retationship to electrocontical state. Am. 1 . Obstet. Gynecol. 153:426-431, 1985

Rigatto $\mathrm{H}_{0}$ Blanco $\mathrm{CE}$, and Walker DW. The response to stimulation of hindind nerites in fetal sheep. in utero, during the different phases of elecrocontical activing. I. Dev. Physiol. 4: 175-185, 1982 .

Rigatro H, Moore M, and Cates D. Fetal breathing and behavior measured phough a donble-mall plexiglas window in sheep. J. Appl. Physiol. 61: 1601-164, 1986.

Rigatto H, Lee D, Davi M, Moore M, Rigato E, and Cates D. Effect of increased arterial $\mathrm{CO}_{2}$ an feral brearhing and behavior in sheep. 1. Appl. Physiol 64: $982-987$. 1988. 


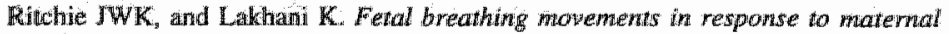
Whalanion of 5\% carbon dioxthe Am. J. Obstet. Gynecol. 136: 386-388, 1980.

Doseriberg AA Response of the cerebral circulation to profound hypocarbia in neondal Lambs. Stroke 191: 1365-1370, 1988.

Ressentrerg AA, Jones MD, Jr, Traystman RV, Simmons MA, and Molteni RA. Response of cerebral blood flow wo changes in $\mathrm{PCO}_{2}$ in fetal, newbom, and adult sheep. Am. J. Ptrysind 242 , $1862+1866,1982$

$\mathbb{R}$ wckeburch $Y_{,}$Gaujour. M, and Eghbali B. Sleep cycles and kinesis in the foetat lamb. Ellectroencephalogr: Clin. Newrophysiol. $42,226-237,1977$.

Rurak DW, and Gruber NC. Increased oxygen consumption associated with breathing acritity in feral lambs: I. Appl. Physiol. 54: 701 707, 1983.

Sack Beaudry M, DeLamater PV, Oh W, and Fisher DA. Umbilical cord cuting triggers hypoertitodothyoninemia and nonshivering thermogenesis in the newborn lamb. Pediatr. Res. 10: 169-175, 1976.

Santiago TV, and Edelman NH. Opoids and breathing. J. Appl. Physiol. 59: 1675-1685, 1985.

Scarpelli EM, Condorelli $\mathrm{S}$, and Cosmi EV. Cutaneous stimulation and generation of breathing in the ferw. Pediatr. Res. 11: 24-28, 1977.

Sheldon MI, and Green $J F$. Evidence of pulmonary $\mathrm{CO}_{2}$ chemosensivity: effects on ventilation. I. Appl. Physiol. 52: 1192-1 197, 1982.

Sherman DJ, Ross MG, Day L, Humme J, and Ervin MG. Fetal swallowing: response to graded maternal hyposemia. J. Appl. Physiol. 71: 1856-1861, 1991.

Sidi D, Kuipers JRG, Heymann MA, and Rudolph AM. Effects of ambient temperature on axygen consumption and the circulation in newborm lambs at rest and during. hypoxemia. Pediatr. Res. 17: 254-258, 1983.

Siesjo BK, and Ingvar M. Ventilation and brain metabolism. Handbook of Physiology.

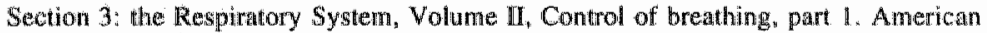
Physiological Society. Bethesda, U.S.A., 1986, p 141\%161.

Sival DA, Visser GHA and Prechtl HH' Fetal breathing mowentents are not a good indicaton of lung developonen after premature rupture of membranes and ollgohydramuios - a preliminary shady. Early Hum. Dew. 28: 133-143, 1992.

Slegel P. Kitagawa H, and Maguire MH. Determinatom of adenosine in felat perfusates of Mushin placental cotyledans using fluorescence derivatization and reversed phase high-performance liquid chromotography. Anal. Biochem. 171: 124-134, 1988.

Sue-Tang A, Brooks AN, Hooper S, White S, Jacobs R, Bocking AD, and Challis JRG. Increased cincalating corticolvophin-releasing homone (CRH) occurs daring fetal hypoxemia with reduced shero-placental blood flow (UBF). but not as a result of maternal hypowema. Meethng of Society of Gynecologic Investigation, St. Lous, MO, Abstract $319,1990$.

Szeto HH. Spectral edge frequency as a simple quantitative measure of maturation of etectrocortical actinity. Pediatr. Res, 27: 289-292, 1990. 
Szeto HH, Cheng PY, Decena IA, Wu D-L, and Dwyer G. Dewelopmental changen in conthaity and stability of breathing in the fetal laww. Am. J. Plysiol. 262. R452-13458. 1992.

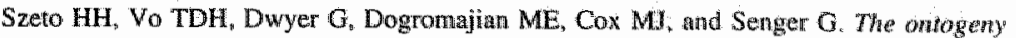
of fetall lamb electrocortical: a power spectral analysis. Am. J. Obstet. Gynecol. 153: $462-466,1985$

Teitel DF, Iwamoto HS, and Rtolph AM. Changes in the pulmonary chalation dwimg birth-related wents. Pediatr. Res. 27: 372,378, 990.

Tiktinsky MH, Hasan SU, Rigawx A, Bishop B, and Morin III FC. The effect of axygenation on breathing movements in the fetal lawb. Pedittw. Res. 31: Abstract 1932, 1992.

Tiktinsky MH, Hasan SU, Rigaux A Bishop B, wnd Morin In FC. Hyperbaric oxygenation increases arousal and breathing mowements in feral lambs. J. Appl Physiol. 77: $902-911,1994$.

Towell ME Johnson J, Smedstad K, Andrew M, and Vu T-L. Fehal blood and trisue $\mathrm{PO}_{2}$ during maternal oxygen breathing. J. Dev. Physiol. 6: 177-185, 1984.

Visser GHA. Poelrman-Weesjes G, Cohen TMN, Bekedam DJ. Feat behator an so wo 32 weeks of gestation. Pediatr. Res. 22: 655-658, 1987.

Vries de JIP, Visser GHA, and Prechtl HFR. The energence ef fetal behawiour. I. Qualitative aspects. Early Hum. Devetop. 7: 301-322, 1982.

Wyas $\mathrm{H}$, Milner $\mathrm{AD}$, and Hopkin IE. Intrathoracic pressure and volume changex during the spontaneous onset of respiration in babies born by cesarean section and by waginal delivery. J. Pediatr. 99: 787-791, 1981.

Walker AM, Oakes GK, threnkranz R, McLaughlin M, and Chez RA. Effects of hypercapnia on uterime and umbilical circularions in conscions pregnant shep. J. Appl. Physiol. 41: 727-733, 1976 .

Walker D. No effect of prostaglandin synthesis inhibition on muscle reflexes in fetal lambs. Am. J. Physiol. 258: R 1213-R1216, 1990.

Walker DW and Davies AN. Effects of hyperhermia on fetal breathmg wowements. J. Dew, Physiol. 8: $485-497,1986$.

Wallen LD, Murai DT, Clyman RI, Lee CH, Mauray FE, and Kittermen IA. Regulation of breathing mowenents in fetal sheep by prostaglandin E2. J. Appl. Physiol.

60: $526-531,1986$

Wallen LD, Murai DT, Clyman RI, Lee CH, Mauray FE, and Kitterman JA Effecti of meclofenamate on breathing movemens in fetal shep before detwery. 1. Appl Physid. 64: $759 m, 766,1988$

Wanatabe T, Kumar P, and Hanison MA. Effects of warm exwironmentol temperatare on the gain of the respiratory chemofiex in the kutren. I. Physicol. 459:336P" 1993.

Wardlaw SL, Stark. RI, Daniel S, and Fantz AG. Effects of hypoxia on beta-enderphin and beta-lipomopin relecte in fetal, newborm, and waternal sheep. Endocrinology 108: $1710-1715,1981$. 
Weening van $H \mathrm{~K}$, Wadmiroff $\mathrm{JW}$, and Raodenburg $\mathrm{PJ}$. Effect of changes in materwat blood gases on feral brearhing movements. Contrib. Gynecol. Obstet. 6: 88-91. 1979.

Wigglesworth JS, and Deswi R: Effects on lung growth of cervical cord section in the rabbit fens. Early Flum, Dev. 3: 51-65, 1979.

Wikening RB, and Meschia $\mathrm{G}$. Fetal axyen uptake, oxygenation, and acid-base balance as a function of werine blood flow. Am. I. Physiol. 244: H749 H755, 1983.

Whiltehouse MW, and Haslam $\mathbb{M M}$. Ability of some antimheumatic drugs to uncouple oxidative phosphorylation. Nature 196:1323in1324, 1962.

Woudstra BR. Aumoudse JG, de Wolf BTHM, Zilstra WG. Nuchal muscle activity at different levels of hypoxemia in fetal sheep. Am. J. Obstet. Gynecol. 162: 559.564, 1990. 


\section{Summary}

Breathing movements are periodically present in utero and must become continuous after birth. Mechanisms involved in the control of breathing in utero and involved in the establishment of continuous breathing at birth were of our interest. All fetuses used in this thesis were instrumented at 128-132 days gestation for recording fetal behaviour and for later connection to an ECMO system to change fetal blood gases, and temperature. In order to observe the mechanism involved at the moment of birth some fetuses were instrumented with a cord occluder (chapter 7 , 8). The following questions were the subject of our investigation.

1. Are breathing movements in utero dependent on the level of $\mathrm{PaCO}_{2}$ ? (chapter 4)

Breathing movements are only present during LV ECOG associated with eye movements. HV ECoG is associated with nuchal muscle activity and there are no breathing movements present. Therefore, breatliing movements have been suggested to be part of the expression of fetal behaviour. Our first objective was to study the mechanism involved in determining the presence of breathing activity in utero by investigating whether the incidence of fetal breathing movements could be affected by the level of $\mathrm{PaCO}_{2}$. During mild hypocapnia the overall incidence of breathing movements, the incidence of breathing movements during LV ECoG and the mean duration of periods of breathing decreased significantly. Fetal ECoG activity showed normal cycling during the periods of mild hypocapnia and the mean duration of LV ECoG periods did not change. During mild hypocapnia, rapid eye movements $(n=3)$ remained associated with $L V$ ECoG and nuchal muscle activity with HV ECOG. These results suggest that the presence of breathing movements in fetal life is not only dependent on the behavioral state but also on the level of fetal $\mathrm{PaCO}_{2}$

2. Does fetal breathing activity respond to increased levels of $\mathrm{CO}_{2}$ ? Does the association of hypercapnia and cooling play a role in the initiation and maintenance of continuous breathing? (chapter 5)

Secondly, we investigated whether the stimulatory response of hypercapnia on fetal breathing is already present in utero and whether 
increased afferent input produced by cooling might change the sensitivity for $\mathrm{CO}_{2}$ owerriding the central inhibition during $\mathrm{HV}$ ECoG. This could shed some light on the mechanisms involved in the initiation of continuous breathing at birth. During fetal hypercapnia frequency, amplitude and incidence of fetal breathing movements during LV ECOG increased significantly compared to isocapnic control on ECMO but it remained absent during HV ECoG. During hypercapnia associated with central cooling there were similar changes in fetal breathing movements during $\mathbb{L V}$ ECoG, however, in 4 out of 7 fetuses fetal breathing movements continued throughout HV ECoG. Hypercapnia associated with centrall cooling can thus override the inhibitory effects of HV ECOG on fetal breathing movements. This may suggest that cooling produced changes in $\mathrm{CO}_{2}$ sensitivity probably by increasing afferent input to the central nervous system.

3. Are the inhibitory effects on fetal breathing during fetal hypoxemia an indirect effect due to the release or production of mediators from the maternal side of the placenta or the ewe? (chapter 6)

Another interesting phenomenon is the observation that fetal hypoxemia inhibits fetal breathing and behavioral activity. The mechanism responsible for this is still under discussion. We examined the possible rolle of maternal or placental substances released or triggered during. hypoxemia which could produce fetal inhibition. There was a decrease in incidence in breathing activity to $21.4 \pm 3.5 \%$, however there was no change in length of breathing periods, only in 7 experiments breathing activity stopped within 7 minutes. Fetal ECoG activity, nuchal muscle activity, rapid eye movements, blood pressure and heart rate were present as normal. We conclude that the normal inhibition of fetal activity during hypoxemia does not seem to be mediated by release of factors from the maternal side of the placenta or the ewe.

4. Does the exclustion of the umbilical circulation and therefore placental modulators play a role in the initiation of breathing at birth? Does a rise in $\mathrm{PaCO}_{2}$ and changes in temperature play a role during this transition? (chapter $7 \& 8$ )

Our final objective was to study the possibility that substances produced by the placenta could control breathing activity and the initiation of continuous breathing activity after birth. It has been speculated that the disuppearance of some theoretically substances produced by the placenta could play a role in the complicated change from periodic to continuous breathing at birth. Breathing movements which occurred after cord occlusion were always associated with LV ECoG. Our results do not support the hypothesis that the initiation of breathing within 5 minutes of birth is dependent on an inhibitory factor of placental origin since breathing activity was not initiated whenever $\mathrm{PaCO}_{2}$ remained constant. Whenever $\mathrm{PaCO}_{2}$ increased, breathing activity was present. These data suggest that a rise in $\mathrm{PaCO}_{2}$ is crucial for the presence of breathing at 
birth. Further, we studied the effect of $\mathrm{PaCO}_{2}$ and temperature in the initiation and maintenance of continuous breathing at birth. After delivering the fetuses in a warm saline bath breathing movements were periodically present. Only after 36-192 min breathing activity became continuously present in all animals $\mathrm{CH} 7.20 \pm 0.04$.

$\mathrm{PaCO}_{2} 7.35 \pm 0.16 \mathrm{kPa}$ and $\mathrm{PaO}_{\mathfrak{z}} 12.78 \pm 2.51 \mathrm{kPa}$ when core temperature had fallen by a mean of $1.2^{\circ} \mathrm{C}$ ). Neonatal breathing activity always stopped by decreasing $\mathrm{PaCO}_{2}$. We conclude that the maintenance of fetal $\mathrm{PaCO}_{2}$ and temperature after cord occlusion delays the establishment of continuous breathing at birth. The level of $\mathrm{PaCO}_{2}$ is crucial in the maintenance of breathing activity.

\section{Conclusions}

1. The presence of breathing movements in fetal life is not only dependent on the behavioral state and determined by the level of fetal $\mathrm{PaCO}_{2}$.

2. During fetall hypercapnia trequency, amplitude and incidence of fetal breathing movements during LV ECOG increased significantly compared to isacapnic control on ECMO but it remained absent during HV ECOG.

3. Hypercapnia associated with central cooling can override the inhibilory effects of HV ECOG on fetall breathing movements.

4. The inhibition of fetal activity during maternal hypoxemia does not seem to be mediated by release of factors from the maternal side of the placenta or the ewe.

5. The initiation of continuous breathing at birth is not dependent on an inhibitory factor of placental origin.

6. Maintenance of fetal $\mathrm{PaCO}_{z}$ and temperature after cord occlusion delays the establishment of continuous breathing.

7. The level of $\mathrm{PaCO}_{2}$ is important in the maintenance of breathing activity in the first tow hours of life. 


\section{Samenvatting}

De ademhalingsbewegingen van een foetus zijn periodiek aanwezig, terwijl deze na de geboorte continue aanwezig moeten zijn. In dit proefschrift wordt beschreven welke mechanismen betrokken zijn bij de ademhaling in utero en tevens welke mechanismen een rol speten bij de overgang van periodiek naar continue ademhaling na de geboorte. Deze mechanismen zijn bestudeerd aan de hand van een onderzoeksopstelling bij foetale Jammeren. Bij een zwangerschapsduar van 128-132 dagen (voldragen zwangerschap 145-146 dagen) werden foetale lammeren geïnstrumenteerd voor registratie van electrocorticale activiteit en electromyografische activiteit van diafragma, oogspieren en nekspieren. Verder werden twee catheters ingebracht ( $a$. carotis en $v_{\text {. jugularis) voor }}$ connectie aan een extra corporaal membraan oxygenatie (ECMO)

systeem. Met gebruik van dit ECMO systeem konden foetale bloedgassen en foetale temperatuur veranderd worden. De invloeden wan variaties in bloedgaswaarden en temperatuur van de foetus op de ademhalingsbewegingen werden bestudeerd. Bij enkele foetale lammeren werd een navelstreng occluder aangebracht om eventuele placentaire factoren die van invloed zouden kunnen zijn van de periodieke naar continue ademhaling na de geboorte te kunnen bestuderen..

Aan de hand van dit onderzoeksmodel werden de volgende vragen bestudeerd:

1. Is de aawwezigheid wan foetale ademhalingsbewegingen afhankelijk wan de arteriêle $\mathrm{pCO}_{2}$ ? (hoofdstuk 4)

Bij de observatie van de foetale lammeren blijkt dat ademhalingsbewegingen in utero alleen aanwezig zijn in combinatic met laag volage hersenactiviteit geassocieerd met de aanwezigheid van oogbewegingen (REM-slaap). Tijdens perioden van hoog voltage hersenactiviteit (non-REM slaap) bestaat er een duidelijke associatie met nekspieractiviteit maar winden er geen ademhalingsbwegingen plaats. In de literatuur wordt daarom gesuggereerd dat de ademhalingsbewegingen een vorm wan gedragsuitdrukking zijn, daar deze alleen tijdens REM slaap optreden en niet tijdens non-REM slaap. Om het effect te metern van de invloed van $\mathrm{pCO}_{2}$ op de ademhalingsbewegingen werd de arteriële $\mathrm{pCO}_{2}$ gevarieerd. tussen normale en matige verlaagde waten, en werden de ademhalingsbewegingen, oogbewegingen, nekspieractiviteit en hersen- 
activiteit geregistreerd. Tijdens de milde hypocapnie (verlaagde $\mathrm{pCO}_{2}$ ) veranderde het gedrag van de foetalle lammeren niet, de electrocorticale activiteit vertoonde een normale cyclus van REM slaap en non-REM slaap, de gemiddelde lengte van periode van REM slaap nam niet af. De oogbewegingen bleven aanwezig tijdens de REM slaap en de nekspier activiteit bleef geassocieend met non-REM slaap. De mogelijkheid om tijdens de REM slaap te ademen bleef hetzelfde. Echter, de incidentie van de ademhalingsbewegingen dalde zowel gedurende de totale tijd en als tijdens de REM slaap. Verder, nam de gemiddelde duur van de lengte van de periode van de ademhalingsbewegingen significant af. Deze resultaten suggereren dat ademhalingsbewegingen niet alleen een gedragsuitdrukking zijn maar dat de aanwezigheid van de foetale ademhalingsbewegingen ook bepaald wordt door de $\mathrm{PaCO}_{2}$.

2. Worden de foetale ademhalingsbewegingen gestimuleerd door hypercapnie? Spelen hypercapnie in combinatie met centrale afkoeling een rol in de initiatie van de continu aanwezige ademhaling na de geboorte? (hoofdstuk 5)

Tijdens experimenten met arteriele thypercapnie (verhoogde $\mathrm{pCO}_{2}$ ) bij de foetus namen zowel de incidentie, de frequentie als de amplitude van de ademhalingsbewegingen toe in vergelijking met metingen die verricht werden tijidens normocapnie (normale $\mathrm{pCO}_{2}$ ). De ademhalingsactiviteit bleef echter een periodiek patroon houden. Deze respons van de ademhaling op hypercapnie blijkt al wroeg in de zwangerschap aanwezig te zijn. Het is beschreven dat de ademhaling continu aanwezig is indien de foetus perifeer afgekoeld wordt. Het is niet bekend of centrale afkoeling een toegenomen afferente input produceert en de gevoeligheid voor $\mathrm{CO}_{2}$ verandert en op deze wijze de inhibitie van de adernhaling tijdens de non-REM slaap doorbreekt. Dit mechanisme zou echter een belangrijke rol kunnen spelen bij het starten van de continue ademhaling ten tijde wan de geboorte. Tijdens hypercapnie in combinatie met centrale afkoeling namen de frequentie, incidentie en amplitude toe. Echter, in 4 van de 7 experimenten waren de ademhalingsbewegingen continu nanwezig, ook tijdens de non-REM slaap. Hypercapnie en centrale afkoeling doorbreken de inhibitie tijdens non-REM slaap. Deze observatie suggereert dat centrale afkoeling veranderingen in de $\mathrm{CO}_{2}$ sensitiviteit veroorzaakt, hoogst waarschijnlijk door een verhoogde afferente input.

3. Wordt de inhibitoire respons op de foetale ademhaling tijdens hypoxemie veroorzaakt door een indirect effect: namelijk het vrijkomen van mediatoren geproduceerd door de moeder of de moederlijke kant van de placenta? (hoofdstuk 6)

Tijdens foetale hypoxemie worden de foetale ademhaling, de oogbewegingen, de nekspier activiteit geinhibeerd. Het mechanisme voor deze inhibitie is niet bekend. $\mathrm{Er}$ is beschreven dat tijdens hypoxemie moederlijke en placentaire hormonen vrijkomen (of worden geproduceerd) die een rol spelen in deze inhibitoire reactie. Tijdens maternale 
hypoxemie en foetale normoxie was er een afname van de incidentie van de foetale ademhalingsbewegingen. Er was echter geen verandering in de lengte van de periode van de ademhalingsbewegingen en in 7 van de 14 experimenten stopten de ademhalingsbewegingen binnen 7 minuten. Ook was er geen verandering meetbaar in de foetale hersenactivitieit, nekspier activiteit, oogbewegingen, bloeddruk en hartslag. Onze conclusie is dat de reguliere inhibitie van de ademhalingsbewegingen tijdens hypoxemie niet veroorzaakt wordt door het vrijkomen van mediatoren geproduceerd door de moeder of de moederlijke kant van de placenta.

4. Spelen bij de initiatie van de continue ademhaling na de geboorte een daling c.q. het wegvallen van door de placenta geproduceerde mediatoren, een toename wan de arteriele $\mathrm{pCO}_{2}$ en een verandering in de omgevingstemperatuur een rol? (hoofdstuk 7 \& 8)

Er werd onderzocht of hormonen c.q. modulatoren geproduceerd door de placenta invloed kunnen hebben op de initiatie van de ademhaling. Hiervoor werd tijdens experimenten de navelstreng geoccludeerd zodat de umbilicale circulatie werd uitgeschakeld en de invloed van de eventuele placentaire hormonen c.q. modulatoren kon worden uitgesloten. Na de navelstreng occlusie kwamen alleen ademhalingsbewegingen voor tijdens laag voltage hersenactiviteit. Deze resultaten steunen niet de hypothese dat de initiatie van de ademhaling na navelstreng occlusie afhankelijk is wan de daling van de plasmaspiegels van inhibitoire

hormonen/modulatoren geproduceerd door de placenta wanneer de $\mathrm{PaCO}_{2}$ gelijk blijft. Wanneer echter de arterièle $\mathrm{pCO}_{2}$ steeg was er wel ademhallingsactiviteit aanwezig. Deze bevinding ondersteunt de stelling dat een stijging in de arterielle $\mathrm{pCO}_{2}$ cruciaal is voor het optreden van ademhalingsbewegingen.

Het effect van $\mathrm{PaCO}_{2}$ en perifere afkoeling werd onderzocht in lammeren geboren in een warm bad gevuld met fysiologisch zout. De temperatuur van het fysiologische zout daalde geleidelijk.

Ademhalingsbewegingen waren periodiek anwezig. Pas dertig minuten tot drie na de geboorte waren de ademhalingsbewegingen in alle lammeren continue aanwezig. Tot die tijd bleven ze periodiek aanwexig. Neonatale ademhalingsactiviteit (continu aanwezige ademhaling) stopte als de arteriẻle $\mathrm{pCO}_{2}$ weer werd verlaagd. Uit deze resultaten kan geconcludeerd worden dat het handhaven van de $\mathrm{PaCO}_{2}$ en temperatusur na navelstreng occlusie de initiatie van de continu aanwezige ademhaling tijdens de overgang van het intra-uterien naar het extra-uterien milieu vertraagt. Het handhaven van de $\mathrm{PaCO}_{2}$ is cruciaal voor het aanwezig zijn van de continue adtemhaling. 


\section{Conclusies}

1. De aanwezigheid van foetale ademhalingsbewegingen in utero is niet alleen een uitdrukking wan gedrag maar is ook athankelijk van de arteriële $\mathrm{pCO}_{z}$.

2. In utero is er een toename van incidentie, frequentie en ampiitude van de ademhalingsbewegingen tijdens hypercapnie. Echter ${ }_{i}$ deze ademhalingsbewegingen blifwen een periodiek patroon houden.

3. Tijdens hypercapnie in combinatie met centrale afkoeling kan foetale ademhaling continu aanwezig zijin, ook tijdens non-REM slaap.

4. De inhibitie van de foetale activiteit tijdens hypoxemie wordt niet veroorzaakt door het vrijkomen van mediatoren geproduceerd door de moeder of door de moederlijke kant van de placenta.

5. De initiatie van de continue adlemhaling na de geboorte is niet afhankelijk van een daling c.q. thet wegvallen van door de placenta geproduceerde mediatoren.

6. Normocapnie en normothermie na navelstreng occlusie vertragen de initiatie van de continue ademhaling.

7. Na de geboorte is het niveau van de $\mathrm{PaCO}_{2}$ belangrijk voor de aanwezigheid van ademhallingsactiviteit. 


\section{Dankwoord}

Jarenlang ben ik in gedachten al een dankwoord aan het schrijwen. Mooie zinnen voor een ieder die mij in goede en slechte tijden heeft ondersteund. Toch kunnen woorden niet optimalal uitdrukken welke gevoelswaarden er in een dankbetuiging besloten zijn voor ieder persoon. afzonderlijk. Ik hoop echter dat ik tijdens mijn promotiejaren, voldoende mijn dankbaarheid heb weergegeven aan alle mensen om mij heen die het mij mogelijk hebben gemaakt dit proefschrift af te ronden. Enkele wil ik hier toch met name noemen:

Prof. Dr. C.E. Blanco, beste Carlos, zonder jouw ideeën, inzet, betrokkenheid en doorzettingsvermogen had hier geen boek gelegen. Voor een goed experiment kwam je uren in het laboratorium zitten of het nu donderdagavond, vrijdagmiddag of zaterdagochtend was. "The best ECMO is boring ECMO", met mooie ademhalingsbewegingen en een goed ECoG. Alle manuscripten waren snel gecorrigeerd en ik ben steeds meer gaan genieten van onze discussies. Ik heb veel van je mogen leren, inhoudelijk maar ook persoonlijk. Carlos, ik ben trots en dankbaar dat ik bij jou AIO beb mogen zijn.

Prof. Dr. M.A. Hanson, dear Mark, your incredible sense of humour and enthusiasm are great. Several times a year we met, sometimes in San Francisco, Toronto or London. Sometimes, in Maastricht, we invited you to one of the wonderful experiments. Even if it didn't happen there were still enough things to analyze, discuss or write about. I enjoyed it always very much, thank you.

Dr. W.J. Maertzdorf, beste Wiel, met veel enthousiasme ben je altijd betrokken geweest bij thet onderzoek. Jij stond altijd voor me klaar, met een schouderklop en een lach wist jij alle problemen weer te relativeren. Verder ben ik onder de indruk wan de nauwgezetheid waarmee je de manuscripten nagekeken hebt.

D.S. de Jong, C.C.P., beste Dick, gedurende het onderzoek ben je altijd betrokken geweest bij wat er gaande was en heb je mij positief gestimuleerd, bedankt.

In het dierenlaboratorium is het allemaal gebeurd. Zonder de liefdevolle: zorg en overgave van May Bost, Joyce Suyk, Frans Slangen, Peter Franssen en Ton van de Bogaard voor de schapen, hadden we nooit zoveel goede experimenten gehad. 
Als ar thjdens een experiment iets niet werkte stond Jan Geilen altijd klaar om "het", wat "het" ook was, te repareren. Jan, we hebben veel plezier gehad.

"Mijn" studenten Han Keunen en Klazina Visser hebben vele uren in het laboratorium doorgebracht, bedankt woor jullie hulp. Han, je bent nu zelf aan het promoveren, het is meer dan alle moeite walard!

Ineke Kilopping, beste Ineke, wij hadden toch als eerste van de afdeling een computer met kleuren monitor! We hebben heel wat uren in het laboratorium gezeten tussen de Jammeren en de schapen. Je nuchtere kijk op de wereld werkte relativerend. 'Mijn schapenmaatje' Harm de Haan was een levend lichtje in duisternis als ik rond middernacht mijn schaap kwam controleren. Tijdens de vrijdagmiddag AIO-club werden de toppen en dalen van het AIO-schap besproken, doctors bedankt!

De kinderartsen, neonatologen en assistenten (we blijwen mountainbiken) van de vakgroep Kindergeneeskunde $(\mathrm{AZM})$ will ik bedanken voor alle interesse, medeleven en aanspraak tijdens mijn promotie-jaren.

Marleen Rosbak, beste Marleen, alle jaren ben jij voor mij een rots in de branding geweest op organisatorisch en persoonlijk wlak. Daarnaast lagen in de branding 2 andere rotsjes, Heidi Bisch en Manon Blaszek, ook jullie bedankt voor alle hulp.

De leden van de beoordelingscommissie wil ik danken voor het nakijken van het manuscript. Bijna alle leden van de commissie heb ik eerder te woord mogen staan tijdens een van de vele buitenlandse congressen waarbij ik verschillende aspecten van mijn proefschrift duidelijk heb mogen maken. Prof. Dr. J. de Haan, ik verheung me erop dat ik nog eenmaal in het openbaar mijn standpunt ten aanzien wan het effect van de navelstreng occlusie op de initiatie van de ademhaling mag bespreken.

In "s Hertogenbosch hebben Karin Hogenbirk, Chris de Kruiff, Ineke Reynen en Wilco Zijlmans mijn werk overgenomer tijdens de ongeplande dagen dat ík onverwacht inspiratie had om altijd het laatste hoofdstuk af te maken.

Drs. R. Beijers, drs. J.H. Hoekstra, drs. C. Jacobs en drs. F. Nabben, de kinderartsen van het Bosch Medicentrum, beste Ruud, Hans, Corrie en Frans $s_{n}$ jullie wil ik bedanken voor het enthousiasme waarmee jullie mij wegwijs maken in de kliniek, nadat ik 5 jaar in het laboratorium gewerkt heb. I. ben erg gelukkig dat het cluster Bosch Medicentrum/AMC

(Prof. Dr. C.J. de Groot, Prof. Dr. P.A. Voûte) mij de kans heeft gegeven om dicht bij huis en haard de opleiding tot kinderarts te volgen.

Sylvia Schoenmakers verzorgde de lay-out van het proefschrift, het is prachtig geworden!

Mijn paranimfen Madelon Ruige en Lilian Kuipers wil ik bedamken voor alle peptalk in afgelopen jaren. Lieve Madelon, je positieve inzet en betrokkenheid aijm ongekend. Ik ben er trots op dat je mijn paranimf bent. 
Mijn vrienden en vriendinnen wil ik bedanken voor het begrip dat "er weer eens aan het proefschrift gewerkt moest worden", betere tijden breken aan.

Mijn dierbare oom Jaap en tarnte Ineke uit het hoge noorden wil ik bedanken voor de jaarlijkse culinaire ondersteuning en de vele attente kaarten en briefjes.

Lieve Lilian, Cristel en Robert, jullie hebben er altijd in geloofd, dit onvoorwaardelijke vertrouwen is voor mij heel belangrijk.

Hans en Elfriede, lieve pappa en mamma, jullie hebben altijd jullie vier kinderen gestimuleerd om te studeren en om onafhankelijk te zijn. Alle vier zijn het gelukkige mensen, ieder op zijn eigen plek. Ik had mijn plek. nooit gevonden zonder jullie steun en vertrouwen. Bedankt.

Mijn hieve Paul, het boek was bijna af, het boek was af, het boek was helemaal af, en het boek was alweer af. Het was niet bij te houden. Zonder jouw nuchtere kijk en positieve steun was het nu zeker niet af geweest. Je leert nu pas de echte Irene kennen.

Kockengen, april 1995 


\section{Curriculum Vitae}

1963

1981

$1981-1988$

$1988-1989$

$1989-1993$

1994 geboren te Sittard op 21 mei.

eindexamen atheneum-B, Henric van Veldeke College, Maastricht

studie geneeskunde, Rijksuniversiteit Limburg, Maastricht

research-fellow perinatologie, Prof.dr. A.C. Bryan en Dr. S.L. Adamson, Mount Sinai Hospital, Research Institute, Toronto, Canada.

assistent in opleiding (AIO), Prof.dr. C.E. Blanco, Academisch Ziekenhuis Maastricht, vakgroep Kindergeneeskunde.

assistent geneeskundige in opleiding (AGIO), vakgroep Kindergeneeskunde, cluster AMC (opleider: Prof.dr. C.J. de Groot) en Bosch Medicentrum (opleider: Drs. J.H. Hoekstra). 


\section{Sponsors}

This study was supported by a grant from the Netherlands Organisation for Scientific Research (NWO).

Financial support by:

Vakgroep Cardiopulmonale Chirurgie en Extra Corporale Circulatie, Academisch Ziekenhuis Mastricht

Vakgroep Kindergeneeskunde, Academisch Ziekentuis Maastricht

Stichting Bevordering Kinderwelzijn

Glaxo BV

Boehringer Ingellheim BV

Friesche Vlag Kindervoeding

Nutricia Nederland BV

Zyma Nederland BV

for the publication of this thesis is gratefully acknowledged. 